\title{
Experimental Investigation of \\ Circumferentially Non-Uniform Heat Flux on the Heat Transfer Coefficient in a Smooth Horizontal Tube with Buoyancy Driven Secondary Flow
}

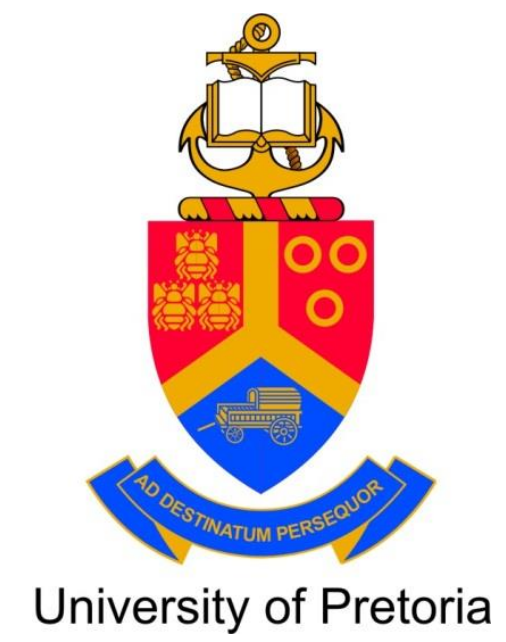

By: W.J.Reid

28236948

Submitted in the partial fulfilment of the requirements of the degree MASTERS OF ENGINEERING in Mechanical Engineering

Supervisors: Prof. J. Dirker and Prof. J.P. Meyer

Faculty of Engineering, Built Environment and Information Technology

University of Pretoria 


\section{Abstract}

Title: Experimental Investigation of Circumferentially Non-Uniform Heat Flux on the Heat Transfer Coefficient in a Smooth Horizontal Tube with Buoyancy Driven Secondary Flow

Author: W.J. Reid

Supervisors: Prof. J. Dirker, Prof. J.P. Meyer

Department: Mechanical and Aeronautical Engineering

Degree: Master of Engineering (Mechanical Engineering)

Most heat transfer tubes are designed for either fully uniform wall temperature or fully uniform wall heat flux boundary conditions under forced convection. Several applications, including but not limited to the solar collectors of renewable energy systems, do however operate with non-uniform boundary conditions. Limited research has been conducted on non-uniform wall heat flux heat transfer coefficients in circular tubes, especially for mixed convection conditions. Such works are normally numerical in nature and little experimental work is available. In this experimental investigation the effects of the circumferential heat flux distribution and heat flux intensity on the single phase (liquid) internal heat transfer coefficient were considered for a horizontal circular tube. Focus was placed on the laminar flow regime of water within a stainless steel tube with an inner diameter of $27.8 \mathrm{~mm}$ and a length to diameter ratio of 72 . Different outer wall heat flux conditions, including fully uniform and partially uniform heat fluxes were studied for Reynolds numbers ranging from 650 to 2600 and a Prandtl number range of 4 to 7 . The heat flux conditions included $360^{\circ}$ (uniform) heating, lower $180^{\circ}$ heating, upper $180^{\circ}$ heating, $180^{\circ}$ left and right hemispherical heating, lower $90^{\circ}$ heating, upper $90^{\circ}$ heating and slanted $180^{\circ}$ heating. Depending on the angle span of the heating, local heat fluxes of 6 $631 \mathrm{~W} / \mathrm{m}^{2}, 4421 \mathrm{~W} / \mathrm{m}^{2}, 3316 \mathrm{~W} / \mathrm{m}^{2}, 2210 \mathrm{~W} / \mathrm{m}^{2}$ and $1658 \mathrm{~W} / \mathrm{m}^{2}$ were applied. Results indicate that the local and average steady state Nusselt numbers are greatly influenced by the applied heat flux position and intensity. Highest average heat transfer coefficients were achieved for case where the applied heat flux was positioned on the lower half (in terms of gravity) of the tubes circumference, while the lowest heat transfer coefficients were achieved when the heating was applied to the upper half of the tube. Variations in the heat transfer coefficient were found to be due to the secondary buoyancy induced flow effect. The relative thermal performance of the different heating scenarios where characterised and described by means of newly developed heat transfer coefficient correlations for fully uniform heating, lower $180^{\circ}$ heating, and upper $180^{\circ}$ heating.

Keywords: Non-uniform heat flux, Nusselt number, Richardson number, horizontal tube. 


\section{Acknowledgements}

I would like to acknowledge the following people for their unwavering support and help throughout the completion of this dissertation.

- Prof. J. Dirker, for his patience, guidance and the hours of help he provided throughout this dissertation.

- Mr A.G. Reid, Mrs S.D. Reid and Miss J.C. Cameron for their endless love and support.

- Mr J. Marsberg for always being available to bounce ideas off of and for the friendship throughout my studies.

I would also like to thank the following institutions for their financial support.

- University of Pretoria.

- National Research Foundation. 


\section{Publications}

The following publication was produced as a result of this work:

REID W, DIRKER J, and MEYER JP, "Experimental Investigation Into the Effect of Circumferential Non-Uniform Heat Flux on a Circular Tube in the Laminar Flow Regime", HEAFAT Conference 2016, Costa del Sol, Spain, Paper Number: 1570249348, 11 - 13 July 2016 


\section{Table of Contents}

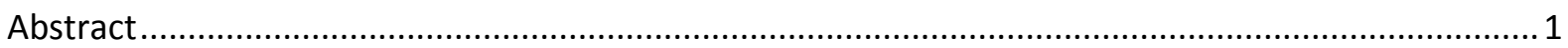

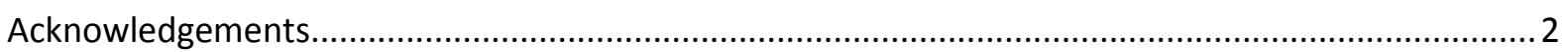

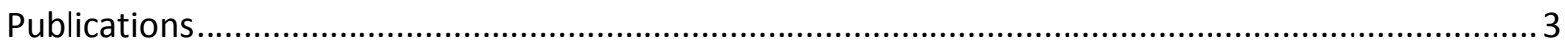

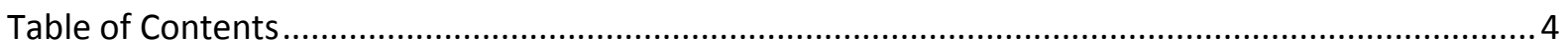

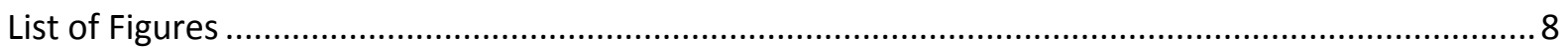

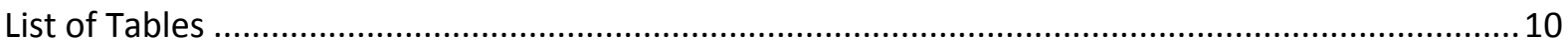

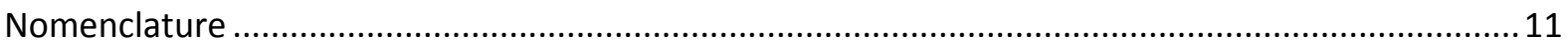

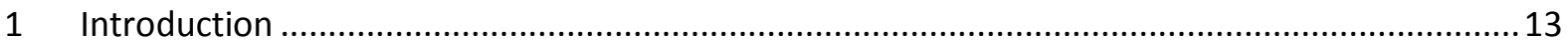

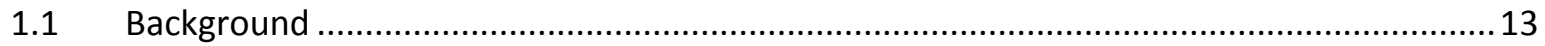

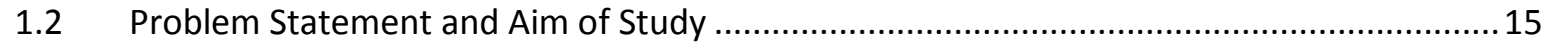

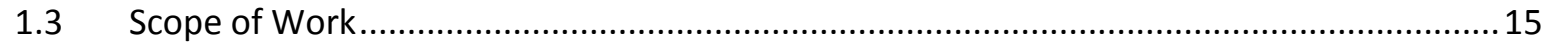

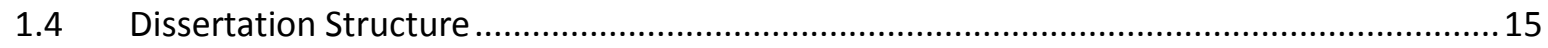

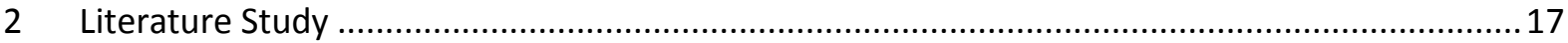

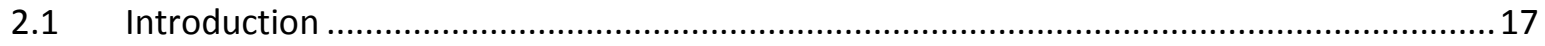

2.2 Important Nomenclature Definitions …......................................................................... 17

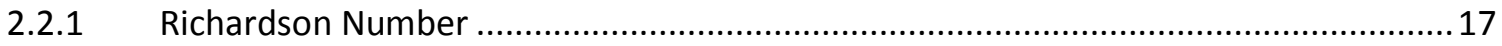

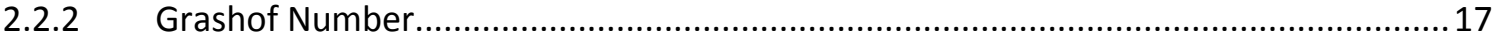

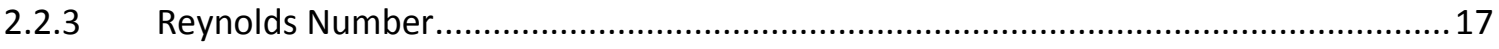

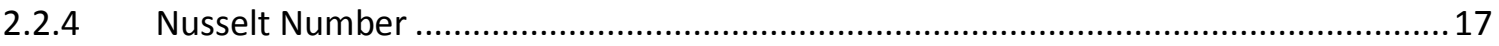

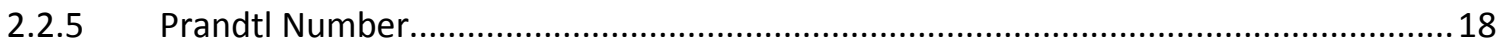

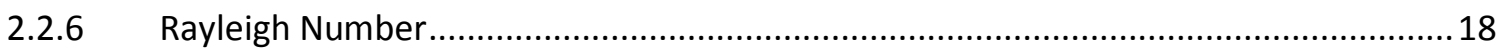

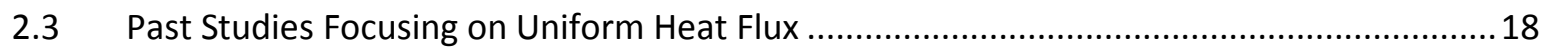

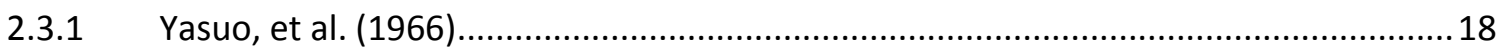

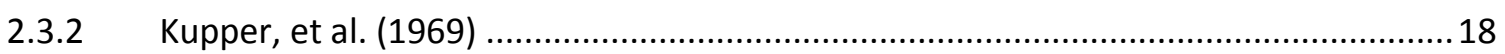

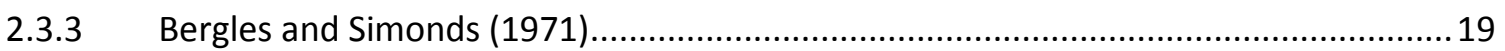

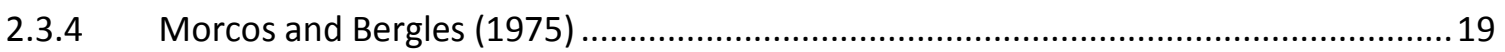

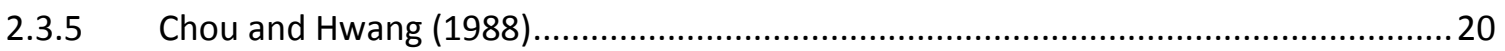

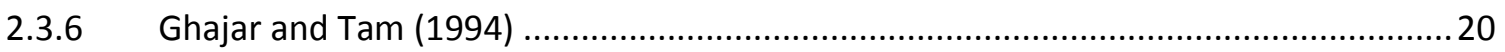

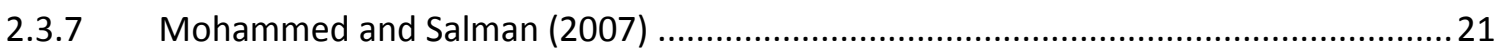

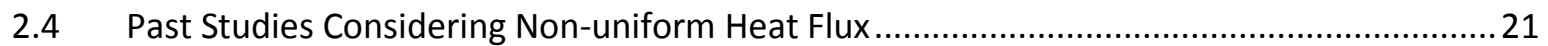

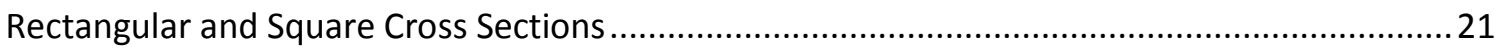

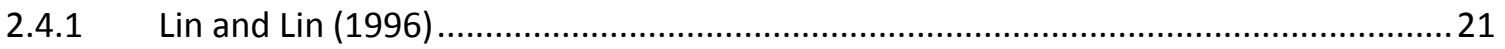

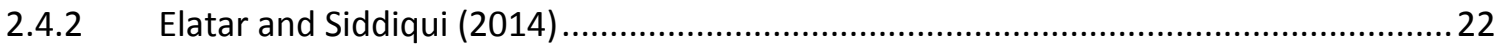

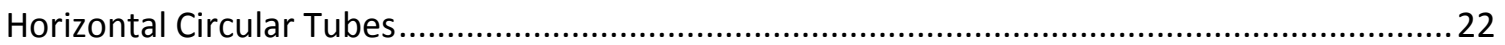

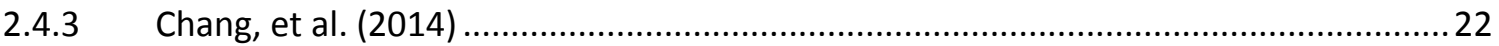




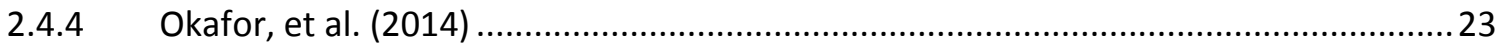

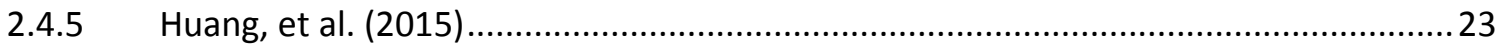

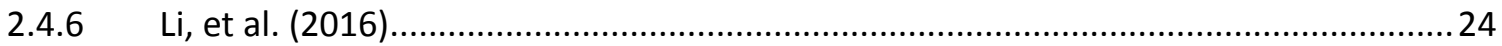

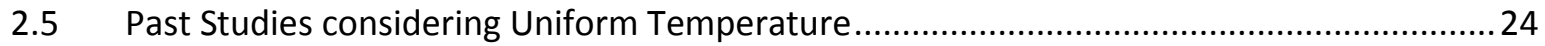

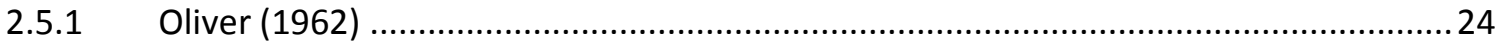

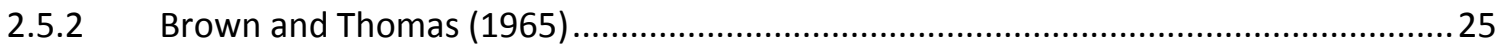

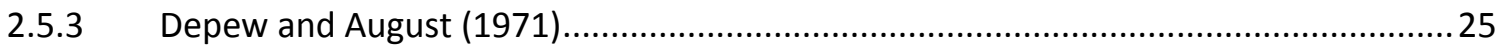

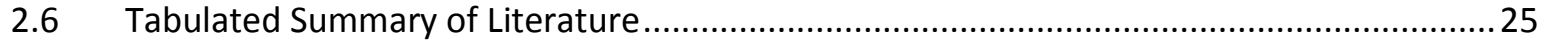

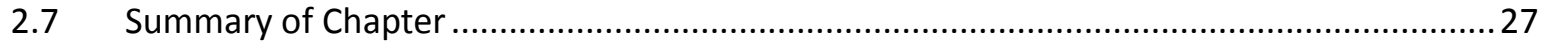

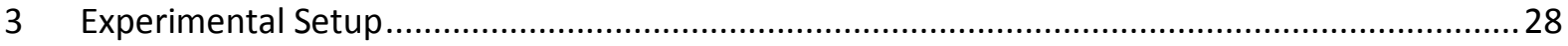

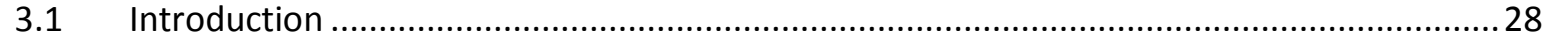

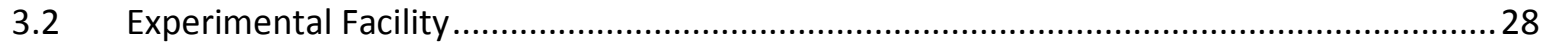

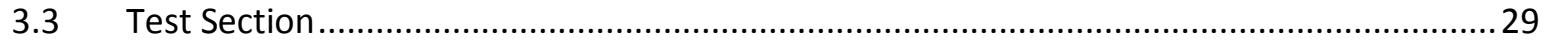

3.3.1 Bulk Fluid Inlet and Outlet Measuring Stations ….....................................................29

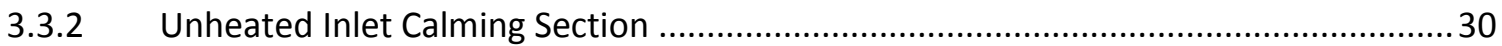

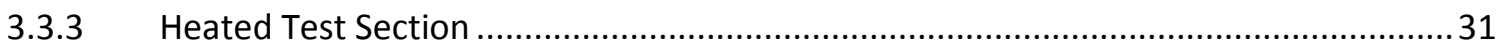

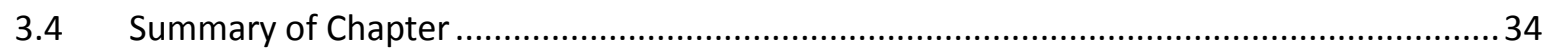

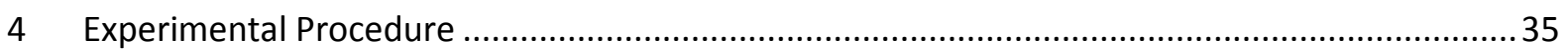

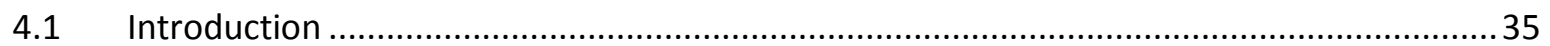

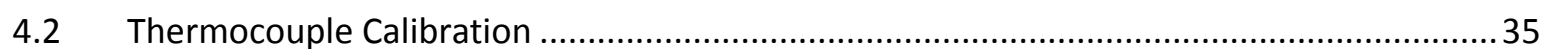

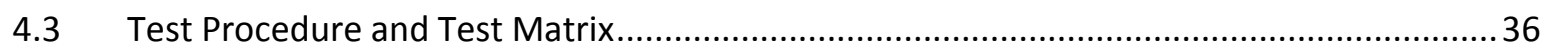

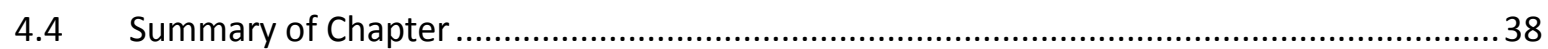

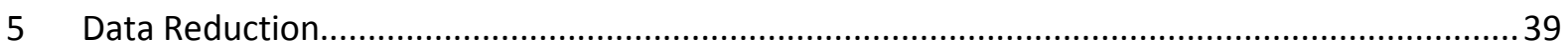

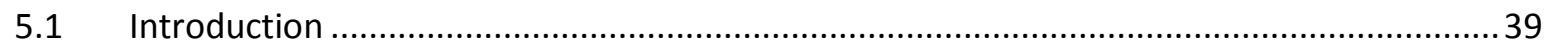

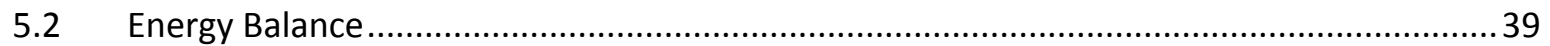

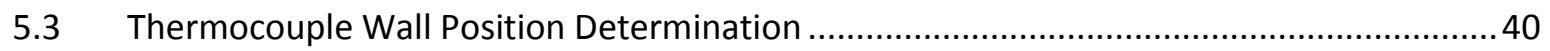

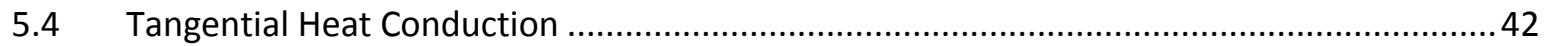

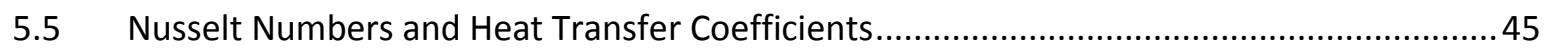

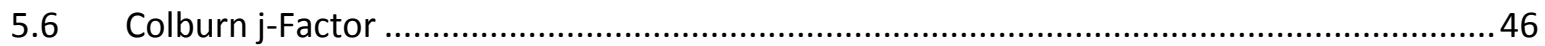

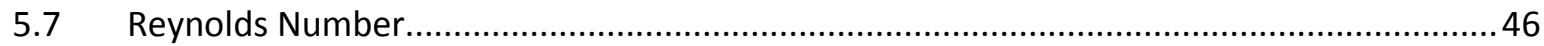

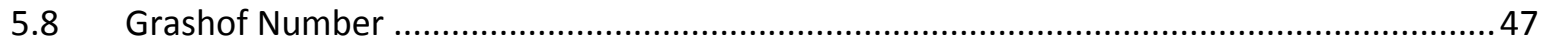

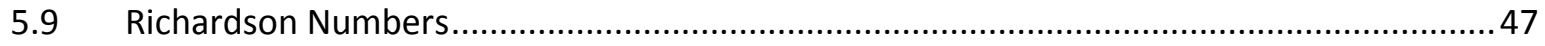

5.10 Uncertainty and Sensitivity Analysis Overview ............................................................ 47

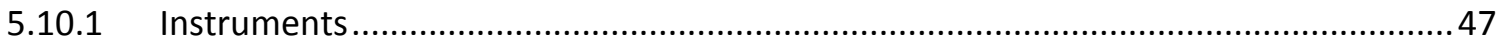

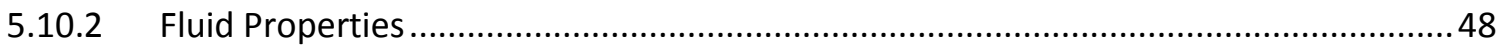

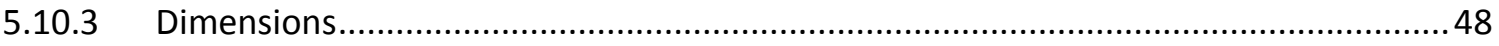




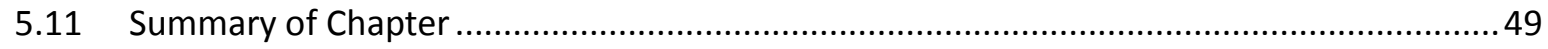

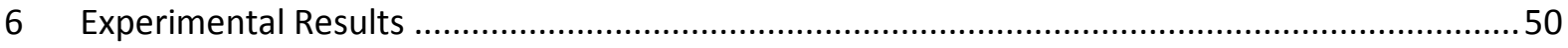

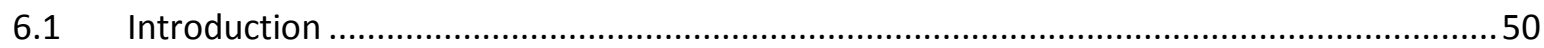

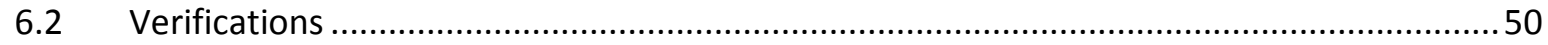

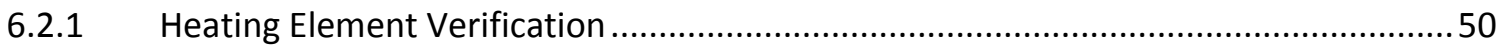

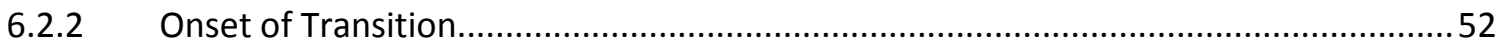

6.2.3 Laminar Comparisons with Correlations from Literature ............................................ 53

6.2.4 Turbulent Comparisons with Correlations from Literature ….......................................54

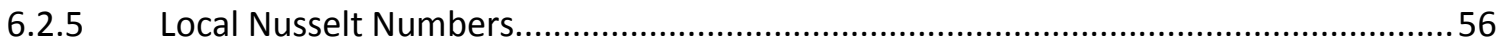

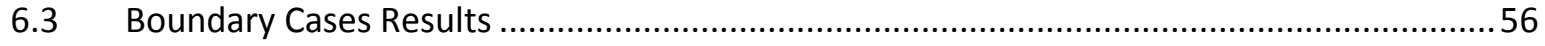

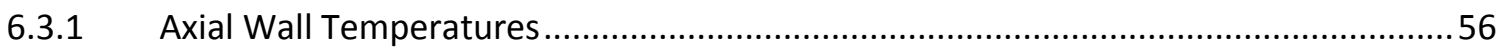

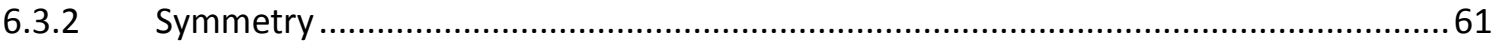

6.3.3 Influence of Heat Flux Position while keeping the Heat Flux Constant ........................61

6.3.4 Influence of Heat Flux Position while keeping the Heat Transfer Rate Constant ..........65

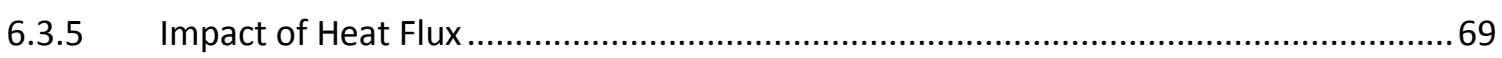

6.4 Effect of the Richardson Number on Nusselt Numbers........................................................ 72

6.5 Effect of Heating Position on Richardson Number ............................................................ 74

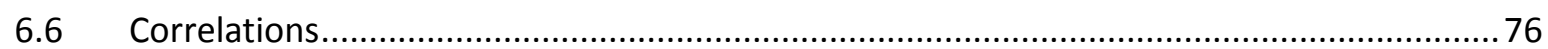

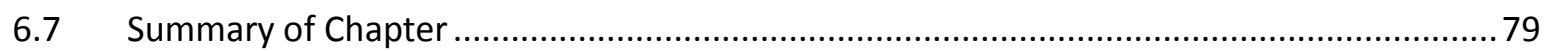

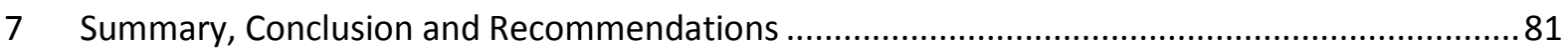

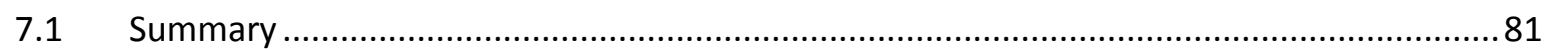

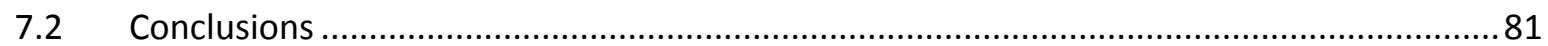

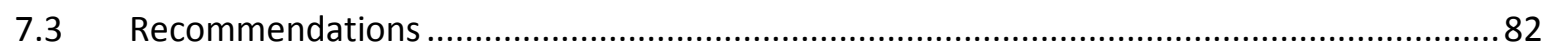

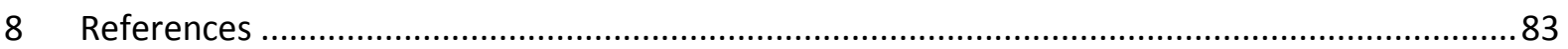

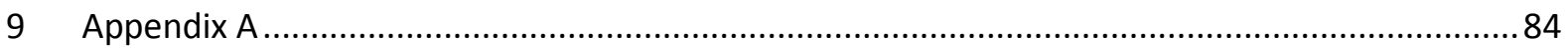

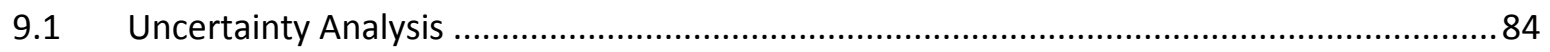

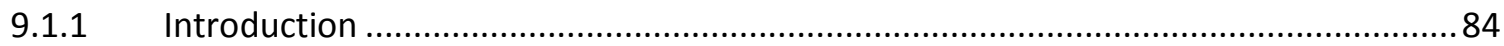

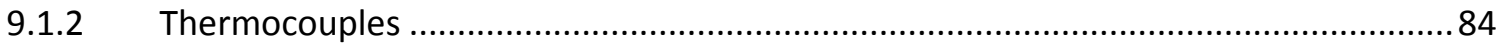

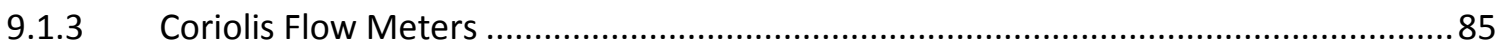

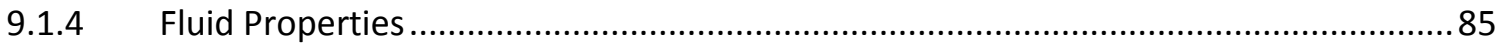

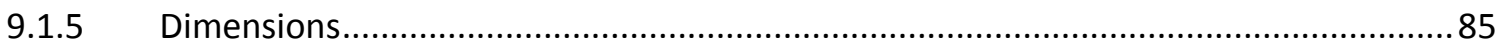

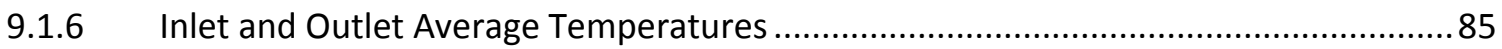

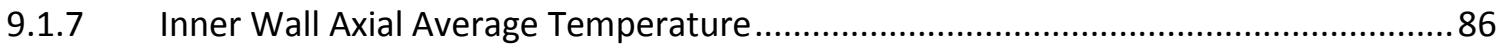

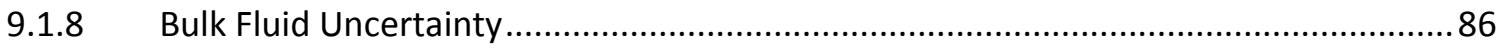

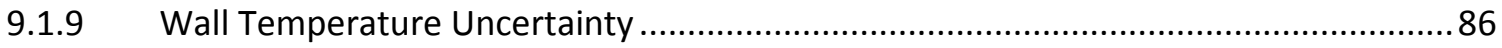




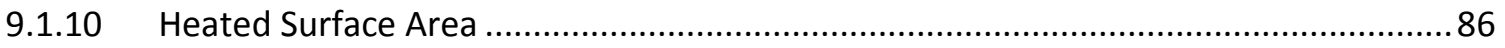

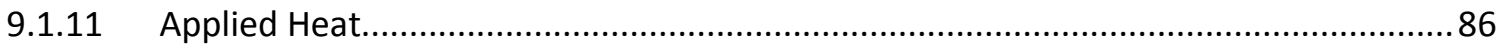

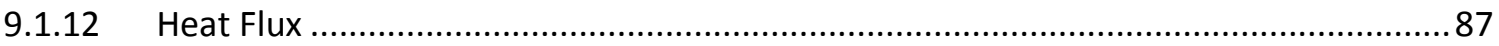

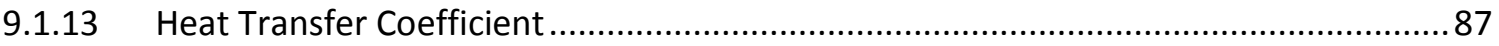

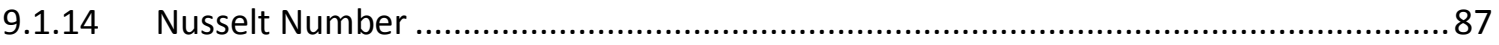

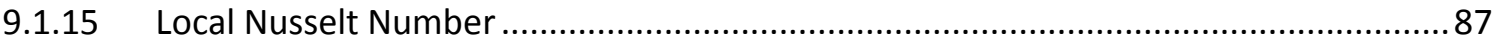

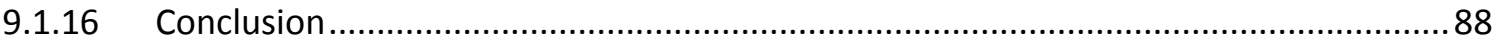

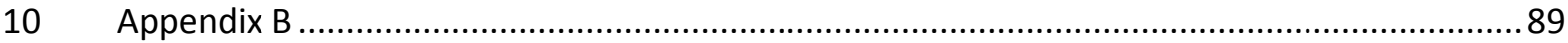

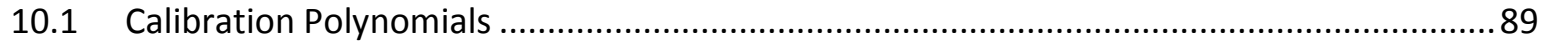




\section{List of Figures}

Figure 1-1: Solar collector layouts: a) Flat plate type collector, b) Concentrated parabolic trough

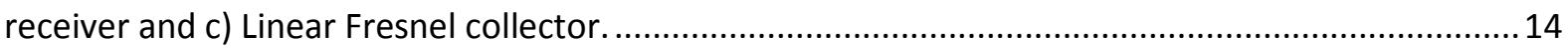

Figure 1-2: Illustration explaining circumferentially non-uniform heat flux and secondary flow ........14

Figure 2-1: Correlation plot for laminar flow of water in a horizontal circular tube with uniform heat flux (Bergles and Simonds, 1971)

Figure 2-2: Secondary flow vertices visualised in the cross section of a rectangular bottom heated duct (Lin and Lin, 1996).

Figure 2-3: Difference between Dittus-Boelter prediction and actual wall temperature distributions (Chang et al., 2014)

Figure 2-4: Comparison of average internal heat transfer coefficients for uniform and non-uniform

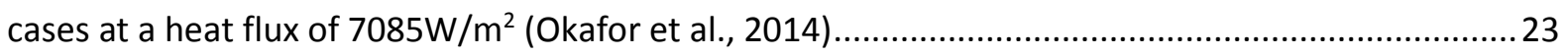

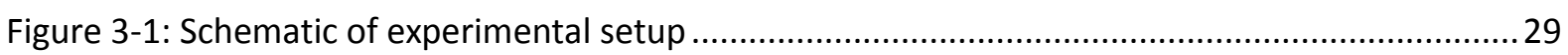

Figure 3-2: Side view schematic of the test section (not drawn to scale) ..........................................29

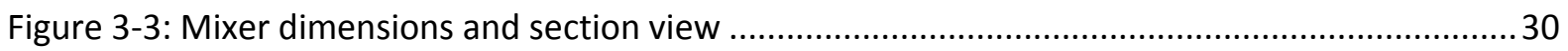

Figure 3-4: Cross section of the connection between the unheated inlet tube and the heated tube. 31 Figure 3-5: Detailed view of the thermocouple tip and heating element placement (not to scale) ....31 Figure 3-6: a) Schematic of a single four-pass heating element strip. b) Photograph of the heating element strips attached to the pipe wall with heat resistant tape, as well as the thermocouple leads

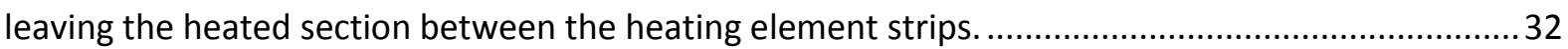

Figure 3-7: Circuit diagram of element connection to DC power supply ............................................ 33

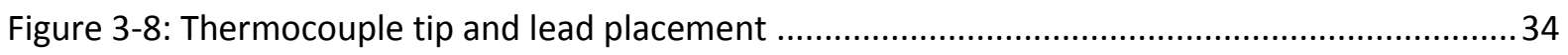

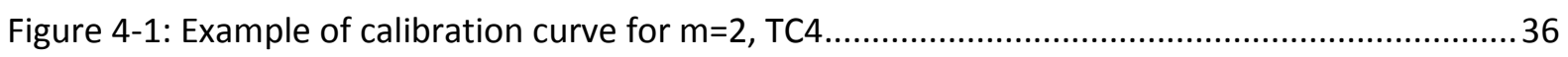

Figure 4-2: Graphical representation of test cases....................................................................... 37

Figure 5-1: Exaggerated actual thermocouple positions and the virtual thermocouple positions due to

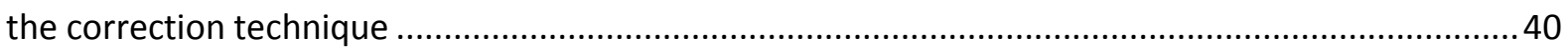

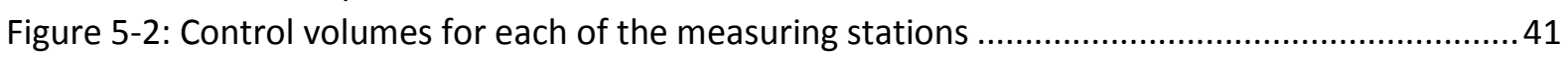

Figure 5-3: Converting the tube wall to a one-dimensional strip and discretising it into different

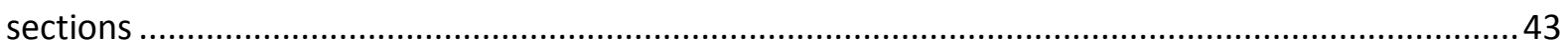

Figure 5-4: Radial heat flux correction percentage graphically represented for cases 5, 6 and $7 \ldots \ldots .45$

Figure 6-1: Axial wetted wall temperature profiles for case 1 ....................................................... 51

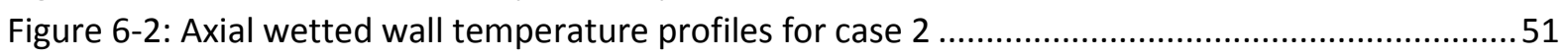

Figure 6-3: Axial wetted wall temperature profiles for case 3 .......................................................52

Figure 6-4: Nusselt number vs. Reynolds number showing the change from laminar to the transitional

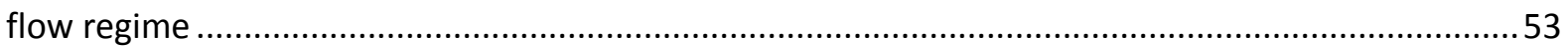

Figure 6-5: Colburn j-Factor vs. Reynolds number showing the change from laminar to the transitional

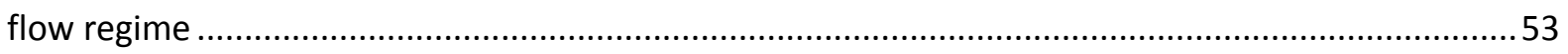

Figure 6-6: Comparison of laminar data from case 1 to previous works............................................54

Figure 6-7: Comparison of turbulent results in case 2 to previous work ...........................................55

Figure 6-8: Local Nusselt number validation for $\mathrm{Re} \approx 900$ and a heat flux of $4421 \mathrm{~W} / \mathrm{m}^{2} \ldots \ldots \ldots \ldots \ldots . . . . .56$

Figure 6-9: Temperature differences between the wetted wall temperatures and bulk fluid

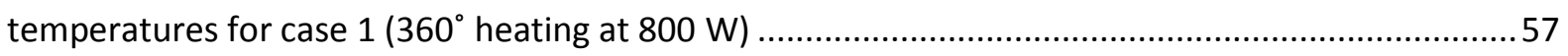

Figure 6-10: Temperature differences between the wetted wall temperatures and bulk fluid

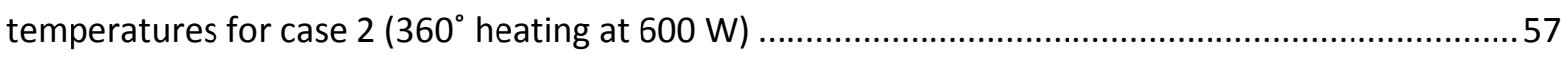

Figure 6-11: Temperature differences between the wetted wall temperatures and bulk fluid

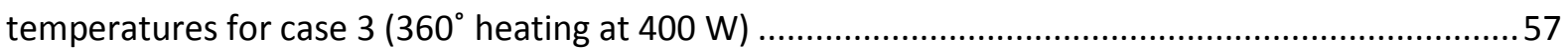


Figure 6-12: Temperature differences between the wetted wall temperatures and bulk fluid

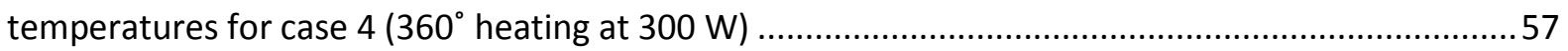

Figure 6-13: Temperature differences between the wetted wall temperatures and bulk fluid

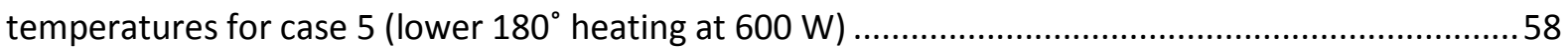

Figure 6-14: Temperature differences between the wetted wall temperatures and bulk fluid

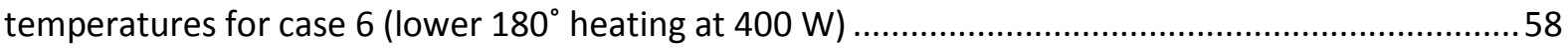

Figure 6-15: Temperature differences between the wetted wall temperatures and bulk fluid

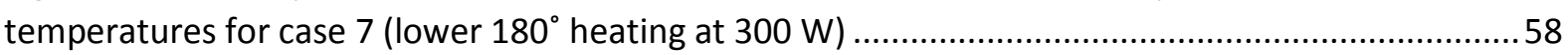

Figure 6-16: Temperature differences between the wetted wall temperatures and bulk fluid

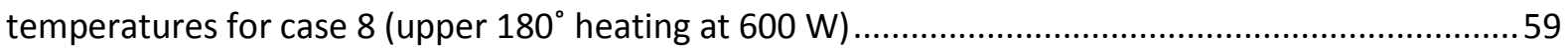

Figure 6-17: Temperature differences between the wetted wall temperatures and bulk fluid

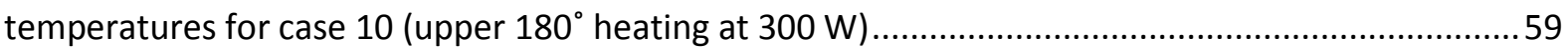

Figure 6-18: Temperature differences between the wetted wall temperatures and bulk fluid temperatures for case 9 (upper $180^{\circ}$ heating at $400 \mathrm{~W}$ ) ...............................................................5 59

Figure 6-19: Temperature differences between the wetted wall temperatures and bulk fluid

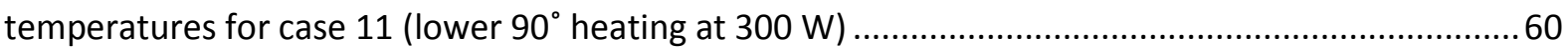

Figure 6-20: Temperature differences between the wetted wall temperatures and bulk fluid

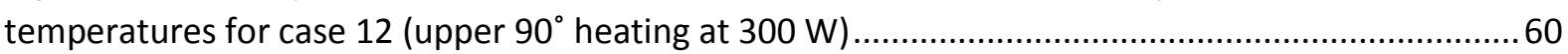

Figure 6-21: Temperature differences between the wetted wall temperatures and bulk fluid

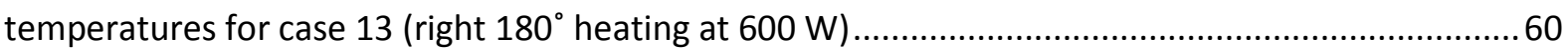

Figure 6-22: Temperature differences between the wetted wall temperatures and bulk fluid

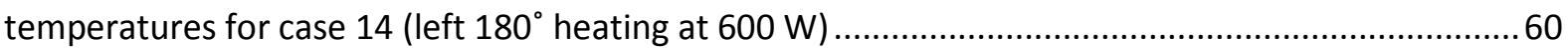

Figure 6-23: Temperature differences between the wetted wall temperatures and bulk fluid

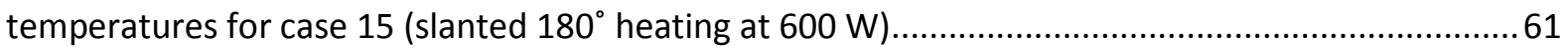

Figure 6-24: Temperature differences between the wetted wall temperatures and bulk fluid

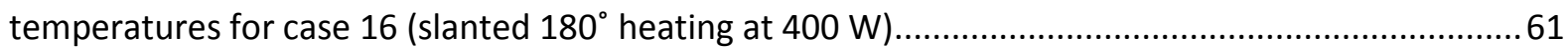

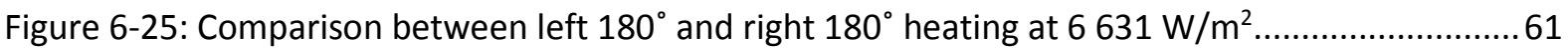

Figure 6-26: Nusselt numbers vs. Reynolds numbers at a heat flux of $6631 \mathrm{~W} / \mathrm{m}^{2}$ for different

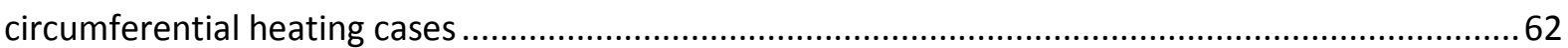

Figure 6-27: Richardson numbers vs. Reynolds numbers at a heat flux of $6631 \mathrm{~W} / \mathrm{m}^{2}$ for different

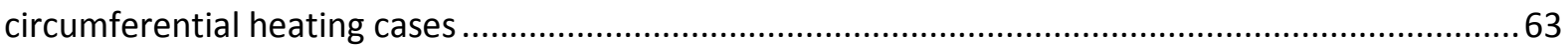

Figure 6-28: Nusselt numbers vs. Reynolds numbers at a heat flux of $4421 \mathrm{~W} / \mathrm{m}^{2}$ for different

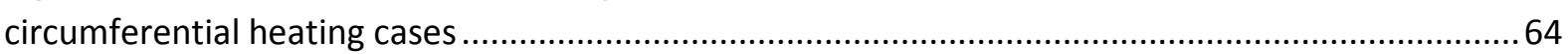

Figure 6-29: Richardson numbers vs. Reynolds numbers at a heat flux of $4421 \mathrm{~W} / \mathrm{m}^{2}$ for different

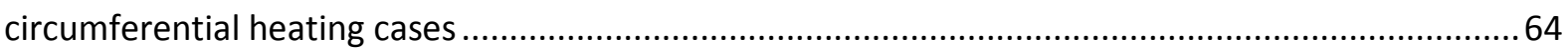

Figure 6-30: Nusselt numbers vs. Reynolds numbers at a heat flux of $3316 \mathrm{~W} / \mathrm{m}^{2}$ for different

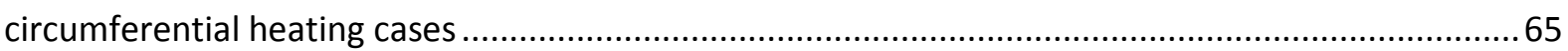

Figure 6-31: Richardson numbers vs. Reynolds numbers at a heat flux of $3316 \mathrm{~W} / \mathrm{m}^{2}$ for different

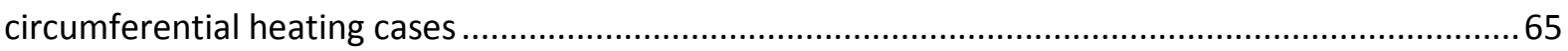

Figure 6-32: Nusselt number vs. Reynolds number for different circumferential heating configurations

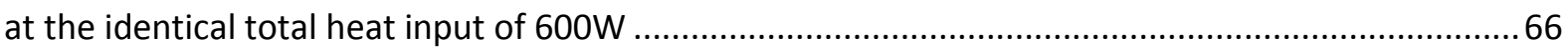

Figure 6-33: Richardson number vs. Reynolds number for different circumferential heating configurations at the identical total heat input of $600 \mathrm{~W}$

Figure 6-34: Nusselt number vs. Reynolds number for different circumferential heating configurations at the identical total heat input of $400 \mathrm{~W}$

Figure 6-35: Richardson number vs. Reynolds number for different circumferential heating configurations at the identical total heat input of $400 \mathrm{~W}$ 
Figure 6-36: Nusselt number vs. Reynolds number for different circumferential heating configurations at the identical total heat input of $300 \mathrm{~W}$

Figure 6-37: Richardson number vs. Reynolds number for different circumferential heating configurations at the identical total heat input of $300 \mathrm{~W}$

Figure 6-38: Nusselt numbers vs. Reynolds numbers for $360^{\circ}$ heating cases at different heat flux's 69 Figure 6-39: Richardson numbers vs. Reynolds numbers for $360^{\circ}$ heating cases at different heat flux's

Figure 6-40: Nusselt numbers vs. Reynolds numbers for lower $180^{\circ}$ heating cases at different heat flux's

Figure 6-41: Richardson numbers vs. Reynolds numbers for lower $180^{\circ}$ heating cases at different heat flux's.

Figure 6-42: Nusselt numbers vs. Reynolds numbers for upper $180^{\circ}$ heating cases at different heat flux's

Figure 6-43: Richardson numbers vs. Reynolds numbers for upper $180^{\circ}$ heating cases at different heat flux's

Figure 6-44: Nusselt number vs Richardson number for $360^{\circ}$, lower $180^{\circ}$ and upper $180^{\circ}$ heating at $\mathrm{Re}=700$

Figure 6-45: Nusselt numbers vs Richardson numbers for the $360^{\circ}$ heating cases at different Reynolds numbers

Figure 6-46: Nusselt numbers vs Richardson numbers for the lower $180^{\circ}$ heating cases at different Reynolds numbers

Figure 6-47: Nusselt numbers vs Richardson numbers for the upper $180^{\circ}$ heating cases at different

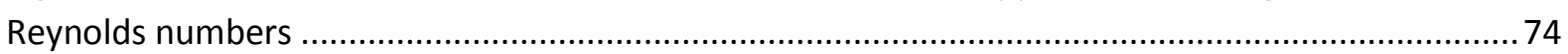

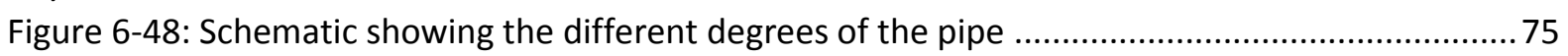

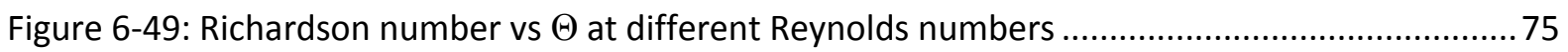

Figure 6-50: Nusselt number vs $\Theta$ at different Reynolds numbers .................................................76

Figure 6-51: Nusselt number vs Richardson number for $360^{\circ}$, lower $180^{\circ}$ and upper $180^{\circ}$ cases....... 76

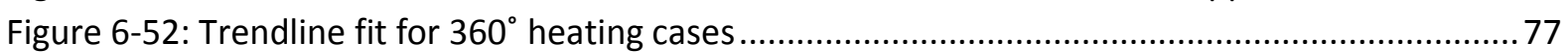

Figure 6-53: Trendline fit for lower $180^{\circ}$ heating cases............................................................... 78

Figure 6-54: Trendline fit for upper $180^{\circ}$ heating cases ................................................................ 79

\section{List of Tables}

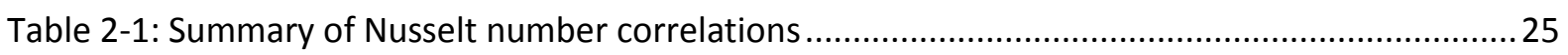

Table 4-1: Test cases with heating degrees and corresponding active heating elements ...................36

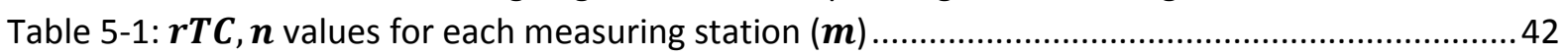

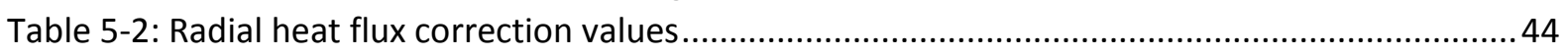

Table 5-3: Uncertainties of the fluid properties (Popiel and Wojtkowiak, 1998) .................................48

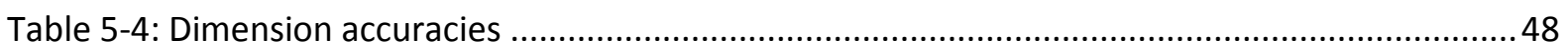

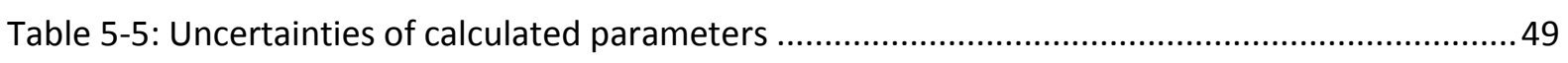

Table 6-1: Inlet and outlet temperature differences for different heating elements ............................50

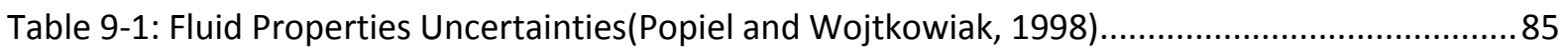

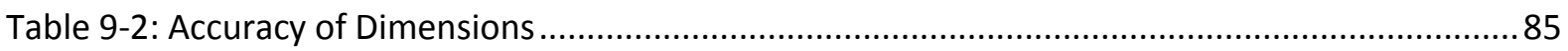

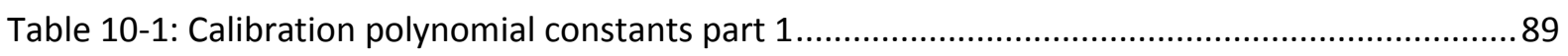

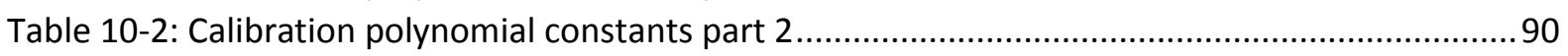




\section{Nomenclature}

$\begin{array}{ll}A & \text { Area }\left[\mathrm{m}^{2}\right] \\ a & \text { Best-fit intercept } \\ b & \text { bias } \\ c & \text { Calibration coefficient } \\ C_{p} & \text { Specific heat }[\mathrm{J} / \mathrm{kgK}] \\ D & \text { Diameter }[\mathrm{m}] \\ g & \text { Gravity }\left[\mathrm{m} / \mathrm{s}^{2}\right] \\ \mathrm{Gr} & \text { Grashof number [-] } \\ \mathrm{Gz} & \text { Graetz number [-] }\end{array}$

$h \quad$ Heat transfer coefficient $\left[\mathrm{W} / \mathrm{m}^{2} \mathrm{~K}\right]$

$\bar{h}$

$h^{*}$

I

$j$

k

$L$

M

$m$

$\dot{m}$

$N$

$n$

$\mathrm{Nu}$

$\overline{\mathrm{Nu}}$

$p$

$p_{p}$

$\operatorname{Pr}$

$P w^{*} \quad$ Modified tube wall parameter

$\dot{Q}$

$\dot{q}$

$r$

$r_{1}$

$r_{2}$

$R$

$\mathrm{Ra}$

$\operatorname{Re}$ [m/K]

Average heat transfer coefficient $\left[\mathrm{W} / \mathrm{m}^{2} \mathrm{~K}\right]$

Hypothetically assumed heat transfer coefficient $\left[\mathrm{W} / \mathrm{m}^{2} \mathrm{~K}\right]$

Electric current $[\mathrm{A}]$

Colburn j-factor [-]

Thermal conductivity [W/mK]

Length $[\mathrm{m}]$

Number of measuring points

Measuring station

Mass flow rate $[\mathrm{kg} / \mathrm{s}]$

Number of data points

Slope of line

Nusselt number [-]

Average Nusselt numbers [-]

Distance between pipes [m]

Precision

Prandtl number [-]

Heat transfer rate [W]

Heat flux $\left[\mathrm{W} / \mathrm{m}^{2}\right]$

Radius [m]

Inner Radius of Insulation [m]

Outer Radius of insulation [m]

Resistivity [K/W]

Rayleigh number [-]

Reynolds number [-]

$$
\begin{gathered}
\mathrm{Gr}=\frac{g \beta\left(T_{s}-T_{B}\right) D^{3}}{v^{2}} \\
\mathrm{Gz}=\frac{D}{L} \operatorname{RePr}
\end{gathered}
$$

$$
\mathrm{Nu}=\frac{h D}{k}
$$

$$
\operatorname{Pr}=\frac{\mu C_{p}}{k}
$$

$$
\begin{gathered}
\mathrm{Ra}=\mathrm{GrPr}=\frac{g \beta\left(T_{s}-T_{\infty}\right) D^{3}}{v^{2}} \operatorname{Pr} \\
\operatorname{Re}=\frac{\rho \mathrm{VD}}{\mu}
\end{gathered}
$$


$\mathrm{Ri}$

$S_{x x}$

$S_{x y}$

$S_{y x}$

$S_{y y}$

$T$

$T_{B}^{*}$

$u$

V

W

$x$

$y$

Z
Richardson number [-]

Sum of squares of $x$

Sum of squares of $x$ and $y$

Standard error of best-fit

Sum of squares of $y$

Temperature $\left[{ }^{\circ} \mathrm{C}\right]$

Hypothetically assumed bulk

temperature $\left[{ }^{\circ} \mathrm{C}\right]$

Calibration coefficient

Voltage [V]

Width [m]

Axial distance [m]

Calibration coefficient

Binary choice (1 or 0) [-]

Greek symbols

$\beta \quad$ Coefficient of thermal expansion

$\delta \quad$ Uncertainty

$\rho \quad$ Density $\left[\mathrm{kg} / \mathrm{m}^{3}\right]$

$\Theta \quad$ Central Heating Angle [ $\left.{ }^{\circ}\right]$

$\mu \quad$ Dynamic viscosity $[\mathrm{kg} / \mathrm{ms}]$

$v \quad$ Kinematic viscosity $\left[\mathrm{m}^{2} / \mathrm{s}\right]$

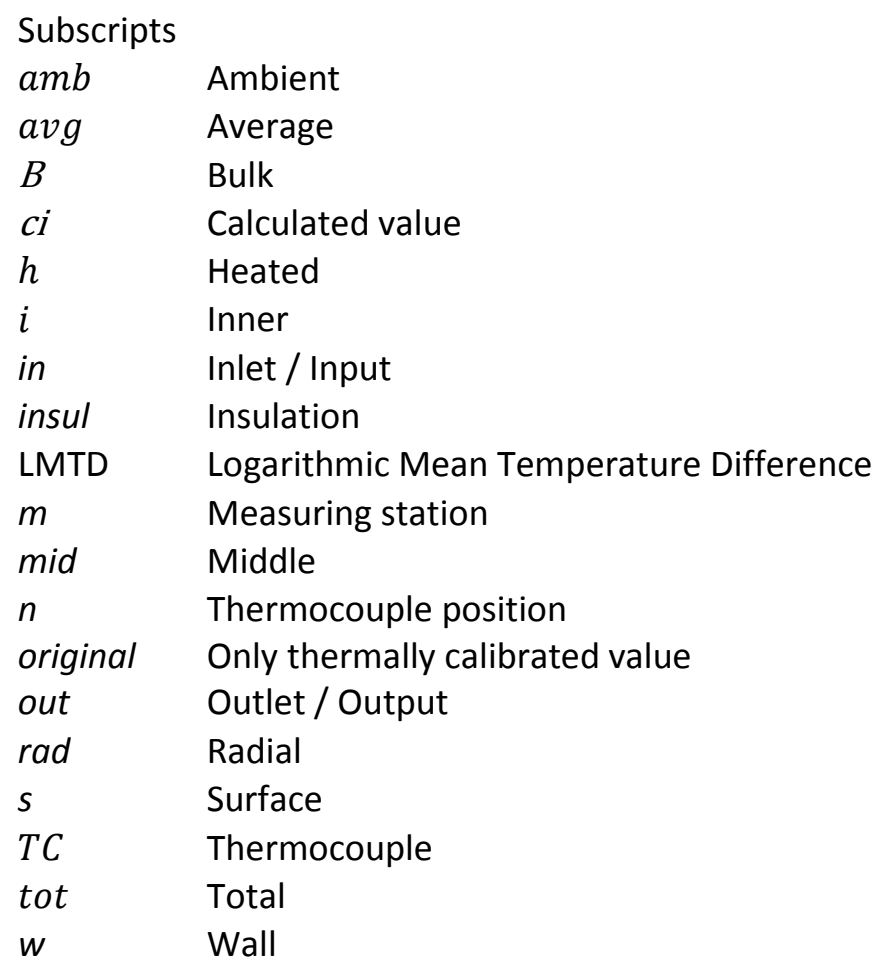




\section{Introduction}

\subsection{Background}

The importance of heat transfer cannot be understated, especially because it is used in almost all industries. Heat transfer, per definition is used to describe the transfer of thermal energy from one medium or region to another. Heat can either be added or removed depending on the application to produce a useful result. Because it is one of the most important energy transfer modes, convective heat transfer has long been the subject of extensive research. Even minor improvements in heat transfer can lead to large improvements in a system's efficiency as a whole.

Circular cross sectional flow passages are widely used due to their advantageous volume to surface ratio, and relatively low manufacturing cost. Therefore, single tubes are often considered during research investigations and subsequently a large number of studies have been conducted on the heat transfer in fluid flow through a tube.

Convective heat transfer is of particular importance when considering fluid based heat transfer. In such circumstances, bulk fluid motion and fluid mixing are often the main means of heat transfer in a system. Therefore, extensive research is continually being performed on convective heat transfer mechanisms and the effect there-of. Often, simplified thermal boundary conditions are considered, such as uniform wall temperature conditions and uniform heat flux conditions. For these cases, thermal and fluid flow performances have been relatively well documented in terms of heat transfer coefficients and friction factors. For circular tubes, specifically, heat transfer coefficients and friction factors have been correlated in terms of the fluid properties, mass flow rate and geometric parameters for both laminar and turbulent flow regime conditions, and to some extent the transition flow regime.

Relatively little research has been performed to investigate the characteristics and the effects of nonuniformity of wall temperature and heat flux conditions on the convective heat transfer coefficient and the friction factor. This is despite the existence of several applications where non-uniform heat flux or non-uniform temperature distributions are present at the heat transfer wall. Such applications include solar energy collector applications and boiler applications. In the solar collector industry for instance, parabolic trough collector tubes, linear Fresnel collector tubes, traditional thermosiphon solar collector tubes and collector tubes in central solar receivers are prone to spatial non-uniform heat flux boundary conditions. Some of these are presented in Figure 1-1 where it can be seen that the thermal incident radiation on the collector tubes is highly directional. In this sense, parabolic trough and linear Fresnel solar collectors use reflectors to direct the solar rays onto the collector tube(s) from below, whereas, traditional thermosiphon solar collectors have tubes which simply receive the incident solar radiation from above. It is thus evident that none of these applications would operate with uniform heat flux or uniform wall temperature profiles. 

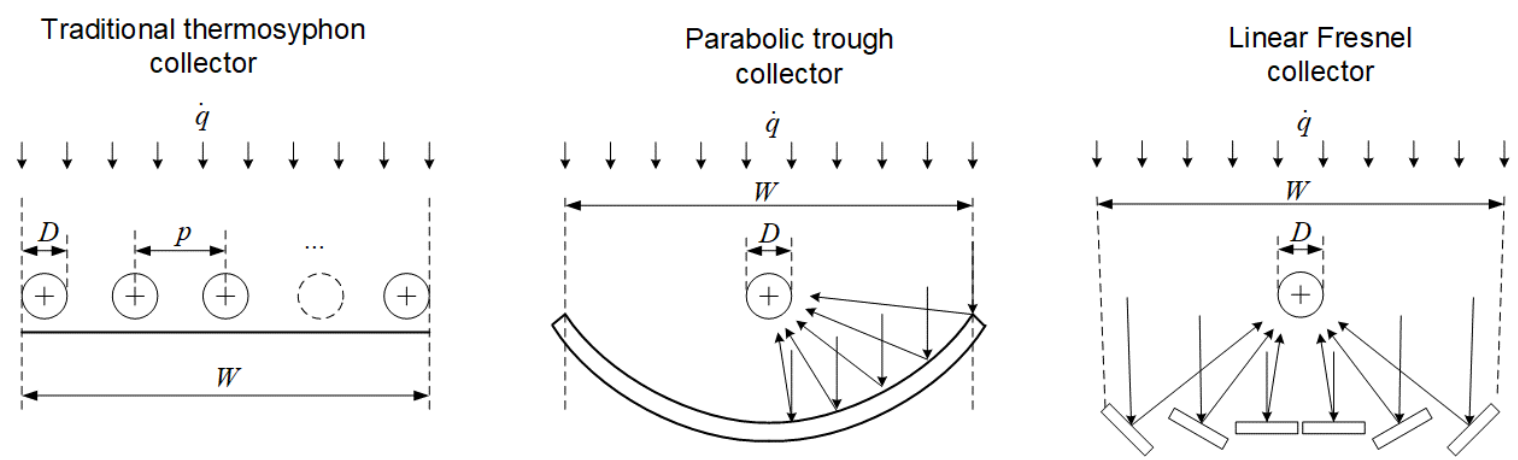

Figure 1-1: Solar collector layouts: a) Flat plate type collector, b) Concentrated parabolic trough receiver and c) Linear Fresnel collector.

For low flow rate operating conditions, which generally are characterized by low Reynolds numbers, non-uniformity of the heat flux can result in a significant uncertainty regarding the convective heat transfer characteristics of even a simplistic geometry such as a horizontal circular tube. This is predominantly due to the presence and the effect of secondary flow effects caused by buoyancy driven flow due to the temperature dependence of the fluid density. The result is the existence of a mixed convection condition, where both the influence of the mechanically forced flow component, as well as the natural convection component must be considered. This is of particular importance in the laminar flow regime where turbulent flow mixing is not present. The effect of mixed convection can result in enhanced heat transfer or suppressed heat transfer depending on the directional components of the forced and buoyancy flow terms. In general the prevalence of mixed convection is often expressed in terms of the Richardson number (the ratio of flow buoyancy to flow shear).

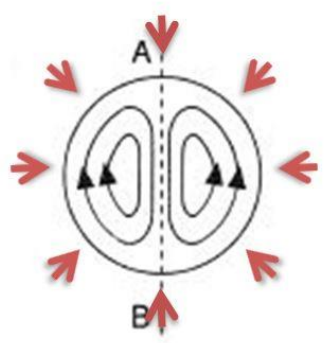

$360^{\circ}$ Heating

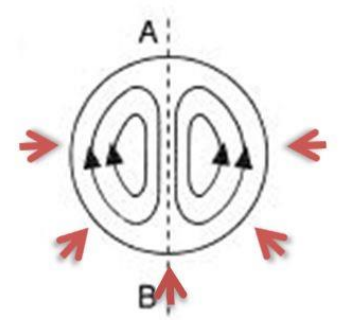

$180^{\circ}$ Heating

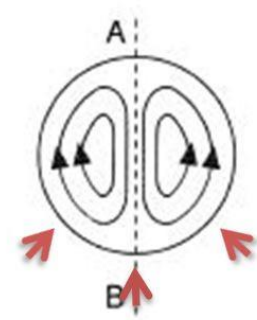

$90^{\circ}$ Heating

Figure 1-2: Illustration explaining circumferentially non-uniform heat flux and secondary flow

Figure 1-2 gives a schematic representation of some different heat flux distributions, as well as possible representations of the plausible buoyancy driven secondary flow pattern that can be found with horizontal heated tubes. The location of the red arrows represent the regions where heat flux could be incident (among many other possible distributions) on the tube wall. Circumferentially uniform heat flux, represented by the $360^{\circ}$ heating distribution, is when the full circumference of the tube receives the same amount of heat per surface area. A tube heated on, for instance, only a $180^{\circ}$ or $90^{\circ}$ angle span of its circumference could still receive a comparable amount of effective heat transfer as the uniformly heated tube (depending on the efficiency of the reflector system). Such cases can be termed circumferentially non-uniform heat flux cases. In all the cases shown in Figure 1-2, the buoyance effects are perpendicular to forced flow direction. 
Relatively little is known about the overall (or average) heat transfer coefficient under such conditions, especially when the heat flux is non-uniform. This is in contrast to the highly turbulent flow conditions where well-developed correlations can be used to predict the Nusselt number. When heating occurs from below, an enhanced heat transfer coefficient can be expected due to the additional mixing effect caused by the secondary flow, however, the magnitude of the increase in the heat transfer performance is not yet well understood or documented. It is also not clear whether factors such as the angle span of the heat flux, the positioning of the heat flux (symmetric or asymmetric to gravity) and the intensity of the heat flux are important to take into consideration. Numerical data can easily be obtained, but little experimental work under controlled conditions has been conducted.

\subsection{Problem Statement and Aim of Study}

There is a need for well-documented experimental studies that investigate the effects of non-uniform heat fluxes on the heat transfer coefficient, particularly in the laminar regime for circular tubes. In terms of the heat transfer performance, a gap in knowledge exists, which if filled, will be valuable to thermal engineers concerned with applications such as solar collectors and boilers which operate at low mass flow rates.

\subsection{Scope of Work}

The intension of this study is to experimentally determine the effective heat transfer coefficient in a smooth horizontal tube exposed to different circumferential heating distributions. Different heat flux configurations, with different angle spans and positions of the incident heat flux onto a tube will be investigated. A $360^{\circ}$ configuration will be used as the reference condition because it is commonly included in literature and is often the default assumption of thermal design engineers. Non-uniform heating configurations with angle spans of either $180^{\circ}$ or $90^{\circ}$ at different heat flux intensities of approximately between $1600 \mathrm{~W} / \mathrm{m}^{2}$ and $6600 \mathrm{~W} / \mathrm{m}^{2}$ will also be investigated for different heating locations around the circumference of the tube. The flow will be contained in a horizontal tube and be kept in the laminar regime $(650 \leq \operatorname{Re} \leq 2600)$. Due to practical limitations, tests will only be conducted on water in the liquid phase. Therefore, steady state single phase convection heat transfer will be considered and not convective boiling heat transfer.

The main objectives of the research will be to:

- complete a comprehensive literature survey

- design and construct an experimental setup suitable to the problem statement and scope

- experimentally perform tests

- provide correlations to describe the convective heat transfer

The experimental investigation will also supply valuable reference data which will be used in future numerical simulation investigations (not within the scope of this project). It is the intension to determine whether the heat flux distribution and the heat flux intensity have a significant influence on the heat transfer coefficient. Once more information is gathered regarding these phenomena, researchers and thermal designers will be able to use this information to investigate and develop more efficient heat transfer systems using the cost effective circular tube geometry.

\subsection{Dissertation Structure}

This dissertation consists of eight chapters. In Chapter 2 a literature review is given to briefly discuss relevant previous work done, as well as fundamental concepts needed to fully understand the work contained in this dissertation. In Chapters 3 and 4 the experimental setup and experimental procedure will be described to give clarity to what was done regarding the testing and how the testing was carried 
out. Chapter 5 contains the data reduction methods used to derive the effective heat transfer coefficients from the experimental data. Chapter 6 contains the experimental results which include the verification of the heating elements, the testing for the presence of transitional flow, and the validation of the laminar, turbulent and local Nusselt numbers. The new results obtained through testing, as well as Nusselt number correlations are also found in Chapter 6. Chapter 7 contains a summary, the conclusions and recommendations for future work. 


\section{Literature Study}

\subsection{Introduction}

In this chapter a brief literature review is presented of works previously conducted on topics that are related to and associated with this study. One particular aspect is that of the thermal boundary which is very important and which can be described either in terms of temperature (uniform or nonuniform), in terms of heat flux (uniform or non-uniform), or in terms of both temperature and heat flux. This dissertation will mainly focus on heat flux boundary condition types, however, reference to other boundary condition types are also made, to place into context the common phenomena at play when buoyancy driven secondary flow is significant. The effects of secondary flow can have different effects on the heat transfer in a system (as mentioned earlier) depending on the directional components of the buoyancy and forced components. For relevance purposes, only buoyancy driven secondary flow perpendicular to a horizontal primary flow will be considered in this investigation.

\subsection{Important Nomenclature Definitions}

\subsubsection{Richardson Number}

In the context of this study, it is important to determine whether natural, mixed or forced convection is present in the fluid flow. As mentioned, the Richardson number (Ri) can be used as a nondimensional guideline for determining which convection type is present in the flow. It is defined as the ratio of the buoyancy force in a fluid and the shear force of the bulk fluid flow. It is commonly expressed as the ratio between the Grashof number and the Reynolds number squared $\left(\mathrm{Ri}=\mathrm{Gr} / \mathrm{Re}^{2}\right)$. When the Richardson number is less than 0.1 the heat transfer can generally be considered as being fully forced convection, while when the Richardson number is above 10 the heat transfer can be roughly classified to be fully natural (free) convection. When the Richardson number is between 0.1 and 10, neither natural nor forced convection can be ignored and mixed convection is present. These are seen as simplified guidelines and are not strict rules.

\subsubsection{Grashof Number}

The dimensionless Grashof number (Gr) is the ratio of buoyancy and viscous forces acting on a fluid. It is often calculated from the thermal fluid properties and the temperature difference in the fluid that is responsible for the buoyancy driven flow. The Grashof number governs the flow regime in natural convection cases.

\subsubsection{Reynolds Number}

The dimensionless Reynolds number (Re) is the ratio of inertial forces to viscous forces for a given flow. It is used as an indication of which flow regime is present. For pipe flow, a Reynolds number of below approximately 2300 indicates laminar flow, a Reynolds number of above approximately 4000 indicates turbulent flow, and a Reynolds number of between approximately 2300 and 4000 indicates transitional flow (transition between bulk laminar and turbulent behaviour).

\subsubsection{Nusselt Number}

The dimensionless Nusselt number $(\mathrm{Nu})$ is the ratio of the heat transferred via convection and the heat transferred via conduction at a boundary between a solid and a fluid. Low Nusselt numbers (close to 1) mean that the heat being transferred is strongly influenced by conduction effects, while for high Nusselt numbers, the heat being transferred occurs mainly due to convection. 


\subsubsection{Prandtl Number}

The Prandtl number (Pr) is a dimensionless number which is the ratio between kinematic viscosity and thermal diffusity. The Prandtl number gives an indication of the relative thickness of the hydrodynamic boundary layer when compared to the thermal boundary layer. Large Prandtl numbers indicate thicker boundary layers.

\subsubsection{Rayleigh Number}

The Rayleigh number ( $\mathrm{Ra}$ ) is another dimensionless number which shows whether the heat within a fluid is being transferred via conduction or natural convection. If the Rayleigh number is below a given threshold the heat transfer is said to be conduction and if the Rayleigh number is above a threshold the heat transfer is natural convection. The Rayleigh number is the product of the Grashof and Prandtl numbers.

\subsection{Past Studies Focusing on Uniform Heat Flux}

\subsubsection{Yasuo, et al. (1966)}

Yasuo, et al. experimentally investigated the buoyancy effects on heat transfer in the flow of air in horizontal tubes. Their work focused on the laminar flow regime ( $\mathrm{Re}=100$ to 13000 ) with a uniform heat flux boundary condition. They showed that the presence of secondary flow increases the Nusselt number and heat transfer coefficient. They discovered that with the secondary flow effect combined with the primary flow caused vortices in the flow with an axis in the horizontal plane and symmetrical about the vertical plane. It was found that the buoyancy effects only start affecting the local Nusselt numbers $(\mathrm{Nu})$ when the product of the Reynolds and Rayleigh numbers (Re $\times \mathrm{Ra}$ ) was higher than $10^{3}$. They proposed a correlation for the Nusselt number in terms of the Reynolds and Rayleigh numbers (Yasuo et al., 1966):

$$
\mathrm{Nu}=0.61(\mathrm{ReRa})^{\frac{1}{5}}\left[1+\frac{1.8}{(\mathrm{ReRa})^{\frac{1}{5}}}\right]
$$

\subsubsection{Kupper, et al. (1969)}

(Kupper et al., 1969) experimentally investigated the effect of free convection on forced convection in a circular horizontal tube with an inner diameter of $5.8 \mathrm{~mm}$ and a length to diameter ratio of a 315 . The Reynolds number range was between 100 and 2000 , while the Grashof number ranged from 300 to 30000 . The working fluid used was water, with a Prandtl number range of between 4 and 9 . Their results showed an increase in the Nusselt number due to the presence of free convection.

They noted that when looking at the local Nusselt number against the axial distance of the heated section, the Nusselt number was at its highest at the beginning of the heated section. The Nusselt number then decreased rapidly along the tubes length, reaching its lowest point at a non-dimensional axial position of $x / D=110$ (where $x$ is the distance from the starting point of the heating section and $D$ is the inner diameter). This coincided to the point where the radial temperature profile in the flow was fully developed.

They also showed that if the Reynolds and Prandtl numbers were kept constant, an increase in the Grashof number resulted in higher Nusselt numbers. The same was found to be true when the Prandtl and Grashof numbers were held constant and the Reynolds number was increased.

When looking at the entrance lengths, they observed that the results were dependant on the Reynolds and Grashof numbers. The flow thermally developed faster with higher Grashof numbers, while the 
flow development was slowed when the Reynolds number was increased. They noticed that for horizontal tubes with a uniform heat flux boundary condition an entry length is needed in order to establish secondary flow.

A correlation was tentatively provided. However, this correlation had a fairly low accuracy. It only correctly predicted $53 \%$ of the data in the thermally fully developed region within $10 \%$.

$$
\mathrm{Nu}=\frac{48}{11}+0.048 \operatorname{Pr}^{\frac{1}{3}}(\mathrm{ReRa})^{\frac{1}{5}}
$$

Thus far it is seen that the proposed Nusselt number correlation put forward by Yasuo, et al. was only a function of the Reynolds and Rayleigh numbers. Kupper, et al. added the Prandtl number to the equation as well as added a term of $48 / 11$. This is roughly equal to 4.36 which is the Nusselt number assumption for fully developed laminar flow under a uniform heat flux boundary condition. The second term is added to compensate for the secondary flow effects present in the fluid.

\subsubsection{Bergles and Simonds (1971)}

Bergles and Simonds experimentally investigated the effects of mixed convection on heat transfer when a uniform heat flux boundary condition is applied. Their tests were conducted in the laminar flow regime for a Reynolds number range of $R e=460$ to 720 . They found that the heat transfer coefficient (Nusselt number) can be greatly affected by the presence of secondary flow and that the heat transfer coefficient can be up to three or four times larger with the presence of secondary flow than when compared to traditional correlations for pure forced convection. While reviewing previous data they observed that the hydrodynamic development length was much shorter for secondary flow cases than when compared to cases where only forced convection was present. They proposed a plot (Figure 2-1) for determining the Nusselt number in the laminar flow regime of water (distilled) in a horizontal circular tube with a uniform heat flux in terms of the non-dimensional axial position and the Rayleigh, Reynolds and Prandtl numbers.

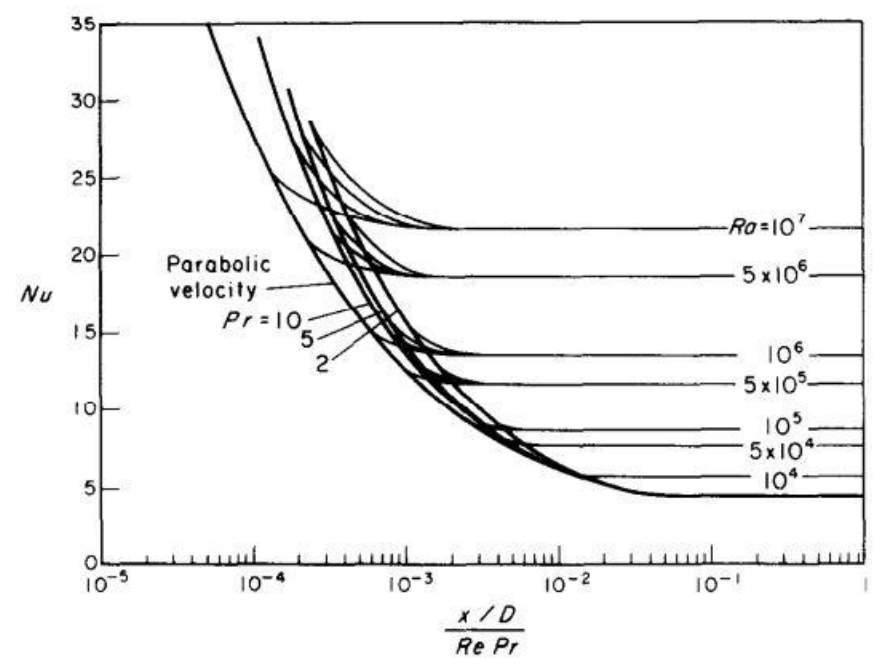

Figure 2-1: Correlation plot for laminar flow of water in a horizontal circular tube with uniform heat flux (Bergles and Simonds, 1971)

\subsubsection{Morcos and Bergles (1975)}

As a follow-on from the earlier effort by Bergles and Simons, Morcos and Bergles experimentally investigated the effects of combined free and forced convection in the laminar regime. They used distilled water and ethylene glycol as their working fluid, uniform heat flux as their boundary condition and horizontal circular pipes as the geometry. Their data indicated that the Nusselt number is 
influenced by the Rayleigh number, variations of the thermo-physical properties of the heat transfer fluid, and the radial conduction in the tubes wall. They proposed a Nusselt number correlation which can be used for a wide variety of fluid properties and flow conditions $\left(3 \times 10^{4}<\operatorname{Ra}<10^{6}, 4<\operatorname{Pr}<175\right.$, $\left.2<P w^{*}<66\right)$ :

$$
\begin{gathered}
\mathrm{Nu}=\left\{(4.36)^{2}+\left[0.145\left(\frac{\mathrm{Gr}^{*} \mathrm{Pr}^{* 1.35}}{P w^{* 0.25}}\right)^{0.265}\right]^{2}\right\}^{1 / 2} \\
P w^{*}=\frac{k D}{k_{w} T}
\end{gathered}
$$

Here the $P w^{*}$ grouping depends on the on the thermal conductivity of the bulk fluid, $k$, the thermal conductivity of the fluid at the wall, $k_{w}$, and the bulk fluid temperature of the fluid, $T$.

The work done by Morcos and Bergles is clearly a continuation of the work done by Bergles and Simons. However, Morcos and Bergles have greatly increased the range of the parameters used in testing, as well as offered a correlation equation as opposed to just a plot for determining the Nusselt number. It can be seen that Morcos and Bergles start with the 4.36 as was used by Kupper, et al. Likewise, the second term is added to compensate for the secondary flow effects present in the fluid.

\subsubsection{Chou and Hwang (1988)}

Chou and Hwang carried out a numerical analysis of the Graetz problem with the presence of natural convection. The Graetz problem is the problem of determining the steady state temperature field of a fluid, when the fluid flows at a different temperature to that of the circular duct it enters. They used the vorticity-velocity method for their analysis. They assumed a tube was heated using uniform heat flux. In order to compensate for the addition of natural convection in the flow, Chou and Hwang used the Boussinesq approximation. This approximation ignores the density differences in the fluid unless gravity is multiplied to the term in the equation. It assumes that the effect of the density differences due to inertia is negligible, whereas the effect of the density differences due to gravity needs to be taken into account. They showed that the secondary flow induced by the natural convection distorts the axial flow and cross-sectional temperature profiles. They numerically showed that the highest fluid velocity and lowest fluid temperature are found at the bottom of the tube.

\subsubsection{Ghajar and Tam (1994)}

Ghajar and Tam experimentally looked at a large range of different conditions from the laminar regime to the turbulent regime (Reynolds range of 280 to 49000 ). Conveniently the paper is broken down into different sections and only the laminar section will be discussed here ( $R e=280$ to 3800 ). They used horizontal circular tubes with water and ethylene glycol as the working fluid. The boundary condition used was a uniform heat flux boundary condition. Their work showed once again the need for an entrance length in order to allow the development of the secondary flow. A correlation was proposed which gave $a+15.4 \%$ and $-16.9 \%$ representation of their experimental data. $86 \%$ of the data was predicted with less than $\pm 10 \%$ deviation:

$$
\mathrm{Nu}=1.24\left((\operatorname{RePr} D / L)+0.025(\mathrm{GrPr})^{0.75}\right)^{\frac{1}{3}}\left(\mu / \mu_{w}\right)^{0.14}
$$

Ghajar and Tam offered a rather complicated correlation for the Nusselt number in the laminar regime. They added the diameter to length ratio $(D / L)$, Grashof number and viscosity ratio $\left(\mu / \mu_{w}\right)$. The viscosity ratio term was added in order to account for the heat flux itself. The presence of the heat flux causes the wall temperature to be greater than the bulk fluid temperature. This temperature 
difference causes the fluid properties to differ from the wall to the center of the flow. The viscosity ratio is added in order to account for these differences.

\subsubsection{Mohammed and Salman (2007)}

Mohammed and Salman experimentally investigated mixed convection on developing flow in circular tubes with a uniform heat flux boundary condition and came to a few interesting conclusions. They found that secondary flow has different effects on the Nusselt number depending on the Reynolds number.

Their study was for a Reynolds number (Re) range of 400 to 1600 , using air as their working fluid. They observed that the secondary flow can decrease the Nusselt number at the lower Reynolds numbers within their range and increase the Nusselt numbers for the higher Reynolds numbers. It was also seen that the Nusselt number increases when more heat flux is applied to the wall. The Richardson numbers (Ri) obtained from their experiments were between 0.13 and 7.125. Since their Richardson numbers were all found to be between 0.1 and 10, their data can be loosely classified as being in the mixed convection range.

They proposed the following correlation for the average Nusselt number as a function of the Rayleigh and Reynolds numbers only (Mohammed and Salman, 2007):

$$
\overline{\mathrm{Nu}}=3.19(\overline{\mathrm{Ra}} / \overline{\mathrm{Re}})^{0.26}
$$

\subsection{Past Studies Considering Non-uniform Heat Flux}

When looking at the previous subsection it can be seen that all the cases are those of uniform heat flux. There has been a large amount of work done regarding heat transfer in circular tube with uniform heating. Unfortunately, this is not the case for non-uniform heating. For that reason, the literature reviewed in this section is somewhat broader than that found in the previous subsection and reference is made to both circular and non-circular flow passages and chambers.

\section{Rectangular and Square Cross Sections}

\subsubsection{Lin and Lin (1996)}

Lin and Lin experimentally investigated the effect of non-uniform (bottom) heating on air flow in a rectangular duct. They looked at low Reynolds numbers ranging from 9 to 186 . They were more interested in the effects that the Reynolds number and Grashof number have on the flow transition of the air, rather than the difference in heat transfer in non-uniform cases as opposed to uniform cases. They showed that the onset of thermal instability occurs closer to the entrance with increased Grashof numbers and decreased Reynolds numbers. Once again, heat transfer enhancement was observed when comparing this case to a purely forced convection cases, and once again, this is attributed to the secondary flow development caused by the buoyancy forces. They also performed flow visualization of the secondary flow vortices, as can be seen in Figure 2-2. 

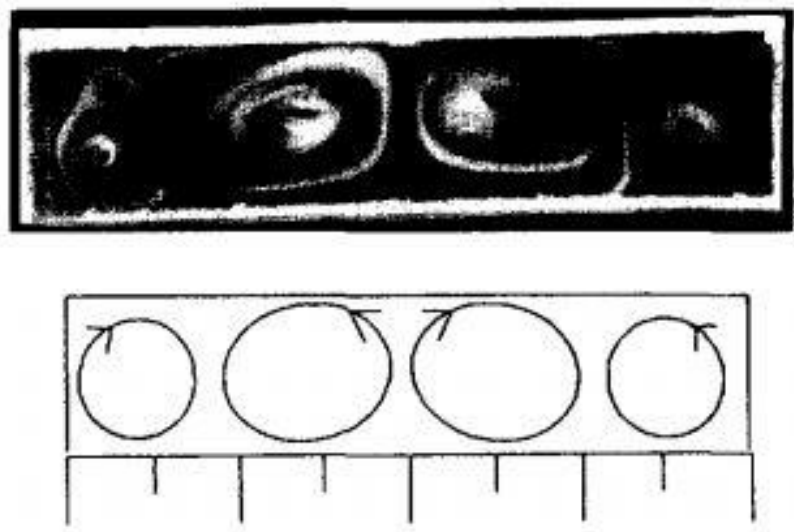

Figure 2-2: Secondary flow vertices visualised in the cross section of a rectangular bottom heated duct (Lin and Lin, 1996)

\subsubsection{Elatar and Siddiqui (2014)}

(Elatar and Siddiqui, 2014) experimentally investigated the effect of bottom heating on the flow of water in a duct with a square cross section. Tests were performed with low Reynolds numbers (300 to $750)$ and high Grashof numbers $\left(6.37 \times 10^{6}\right.$ to $\left.3.86 \times 10^{7}\right)$. They showed that the secondary flow was dependent on the Reynolds and Grashof numbers and that turbulence intensity can be increased due to secondary flow. This turbulence is brought about when the rising plumes (caused by the thermal buoyancy effect) of warm fluid interact with each other and the shear flow. Under the correct conditions it was seen that the flow effect of the bottom heating can have three dimensional effects, with a back flow along the top unheated portion being observed when Ri $>55$. The back flow intensified with an increase in wall temperature.

\section{Horizontal Circular Tubes}

\subsubsection{Chang, et al. (2014)}

Chang, et al. conducted both experimental and numerical tests on the heat transfer characteristics of non-uniform heat flux on a solar thermal absorber tube. Their tests were for flow in the turbulent regime $\left(\operatorname{Re}=1 \times 10^{4}\right.$ to $\left.3.5 \times 10^{4}\right)$, with water used as the working fluid. Only the upper $180^{\circ}$ of the circular tube was heated in the tests.

It can be seen that the Dittus-Boelter correlation still holds true for both uniform and non-uniform heating turbulent cases. This is due to the fact that forced convection is the main mode of heat transfer in turbulent flow and the free convection is almost negligible. This is why more extensive research is needed with regard to non-uniform heat flux in the laminar regime, where mixed convection in more prevalent.

Figure 2-3 shows the wall temperature distributions for a selected cross section of the tube. 


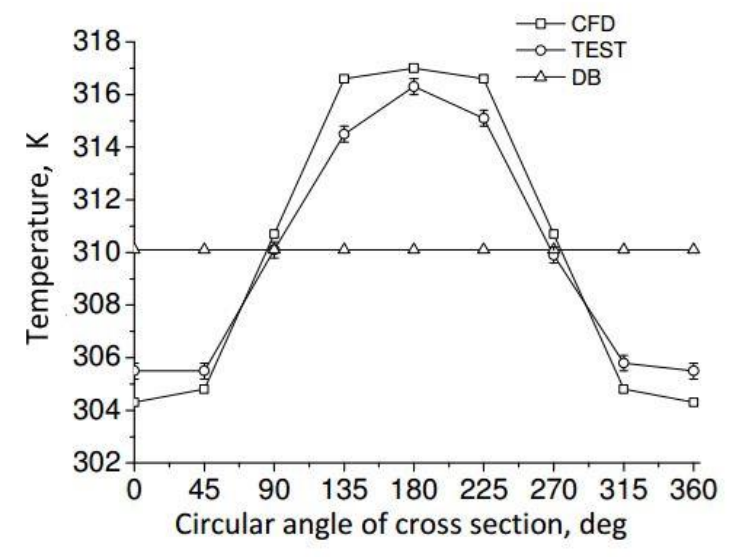

Figure 2-3: Difference between Dittus-Boelter prediction and actual wall temperature distributions (Chang et al., 2014)

\subsubsection{Okafor, et al. (2014)}

The Okafor, et al. considered circumferentially non-uniform heat flux distribution in the laminar flow regime of a tube. A length ration $(L / D)$ of 159 was used, with a Reynolds number $(\mathrm{Re})$ range of 180 to 2 200.The heat flux was applied as a sinusoidal function around the heated section of the tube.

It was observed that the average internal heat transfer coefficient increased as the Reynolds number increased. This was true regardless of the uniformity of the heat flux. It was also noted that both the average and axial local heat transfer coefficient were larger in the non-uniform heat flux cases. When $L / D$ was increased there was seen to be an increase in the average and axial local heat transfer coefficients as well as an increase in the thermal efficiency of the system. Figure 2-4 shows the increase in the average internal heat transfer coefficient when non-uniform heat flux is applied.

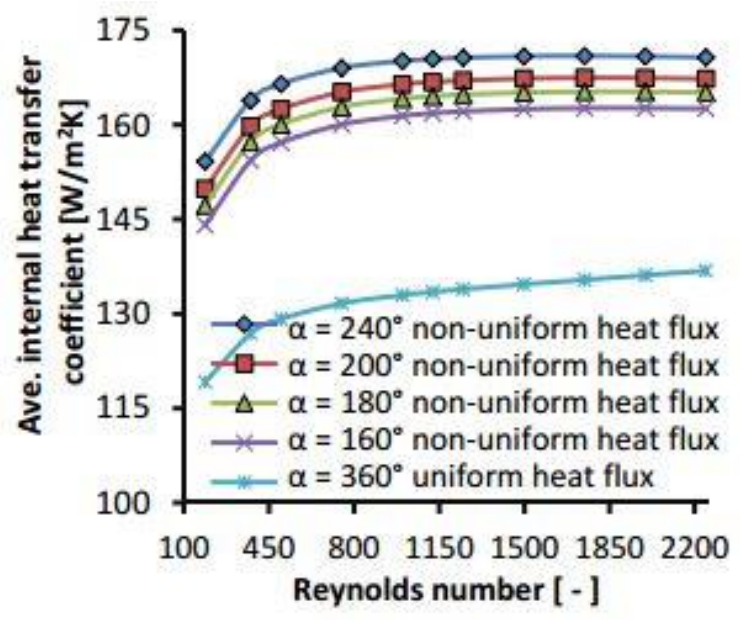

Figure 2-4: Comparison of average internal heat transfer coefficients for uniform and non-uniform cases at a heat flux of $7085 \mathrm{~W} / \mathrm{m}^{2}$ (Okafor et al., 2014)

\subsubsection{Huang, et al. (2015)}

(Huang et al., 2015) performed a numerical study on the effects of mixed convection for fully developed flow in a circular tube with a non-uniform heat flux boundary condition. The numerical model and heat flux distribution were modelled on a parabolic trough solar collector. Their numerical model allowed for either the top half of the horizontal tube to be heated (non-uniform heating) or for the entire pipe to be heated (uniform heat heating). The numerical study was performed for flow in the turbulent regime ( $\mathrm{Re}=2 \times 10^{4}$ to $10^{5}$ ) and used super-heated steam as the working fluid which had a Prandtl number of approximately 1.5 . 
When comparing uniform heat flux cases and non-uniform heat flux cases they observed a marked difference between the velocity fields, temperature distribution, flow resistance and heat transfer. It is noted that the Nusselt number is larger for the non-uniform heat flux cases.

\subsubsection{Li, et al. (2016)}

(Li et al., 2016) performed a numerical investigation on fully developed mixed convection in a parabolic trough solar receiver tube in the laminar flow regime. Their study used super heated steam as the working fluid, and the Boussinesq approximation was used.

They tested $180^{\circ}$ heating spans applied to the left hand side of the tube, 2 slanted cases of varying degrees (still applying $180^{\circ}$ heating) and a lower $180^{\circ}$ heating case. They also tested a uniform heating case to use as a reference. A Reynolds range of $250<\operatorname{Re}<1000$ and a Grashof range of $0<\mathrm{Gr}<3.2 \times 10^{6}$ was used.

It was found that due to the effects of the buoyancy forces, the stream wise flow moves downwards (gravitationally speaking) which causes the formation of a high speed region near the bottom of the receiver tube. The fluid with higher temperature moves upward along the tube wall, while the cooler fluid in the middle flows downward creating vortices. The strength of the vortices are seen to be greater in cases of non-uniform heat flux when compared to cases with uniform heat flux boundary conditions.

They showed that the intensity of the secondary flow increases as the Grashof number increases when the lower $180^{\circ}$ of the pipe is heated. It is also seen that the Nusselt number rose sharply when the Grashof number was increased for the lower $180^{\circ}$ heating case.

They showed that when the Grashof number is greater than 12500 the natural convection can improve the heat transfer rate by $10 \%$ when compared to purely forced convection.

\subsection{Past Studies considering Uniform Temperature}

Referring to some past works, including uniform temperature is deemed necessary, even though uniform temperature will not be investigated in this study. This is because uniform temperature profiles are the other major boundary condition where secondary flow is present. Only the fundamental cases of uniform temperature profiles will be looked at here, as they are deemed the most important, as the uniform temperature studies diversify greatly the closer you get to present day. They differ in terms of geometry, surface roughness, working fluid type and many other facets which are not important to this study.

\subsubsection{Oliver (1962)}

Oliver experimentally investigated the effect of natural convection on the heat transfer on a number of working fluids in horizontal tubes. He used pure glycerol to standardize his experimental setup, and thereafter conducted the tests on water, ethyl alcohol and a mixture containing approximately $80 \%$, by weight, glycerol in water. The Grashof and Prandtl ranges were as follows, $29<\mathrm{Gr}<1.6 \times 10^{5}$ and 1.9 $<\operatorname{Pr}<326$. The tests were performed in the laminar flow regime and although the Reynolds number range is not explicitly stated, it is indicated that the highest Reynolds number reached was 1500 with most of the tests being performed at much lower Reynolds numbers. Oliver proposed a correlation to predict the Nusselt number (Oliver, 1962). This correlation makes use of the Graetz number (Gz), which is used to characterise the laminar flow. It represents the time taken for heat to be transferred radially into a fluid by means of conduction. 


$$
\mathrm{Nu}\left(\frac{\mu_{\mathrm{w}}}{\mu_{B}}\right)^{0.14}=1.75\left(\mathrm{Gz}+5.6 \times 10^{-4}\left(\operatorname{GrPr}\left(\frac{L}{D}\right)\right)^{0.7}\right)^{\frac{1}{3}}
$$

\subsubsection{Brown and Thomas (1965)}

Brown and Thomas continued the work carried about Oliver, focussing their experimental work on the effect of combined forced and free convection on heat transfer of water. Horizontal tubes were used and the experiments were carried out in the laminar regime. The correlation proposed by Brown and Thomas gives and accuracy of $\pm 8 \%$ for cases where water is used as the working fluid. The given correlation can be seen to be very close to that of Oliver's with the secondary term being the only change (Brown and Thomas, 1965).

$$
\mathrm{Nu}=1.75\left(\frac{\mu_{\mathrm{B}}}{\mu_{w}}\right)^{0.14}\left(\mathrm{Gz}+0.012\left(\mathrm{GzGr}^{\frac{1}{3}}\right)^{4 / 3}\right)^{\frac{1}{3}}
$$

\subsubsection{Depew and August (1971)}

Depew and August performed and experimental investigation on the heat transfer due to combined free and forced convection in horizontal tubes. The tests were performed in the laminar flow regime with the highest Reynolds number being 1800 . Three different fluids were used in the testing, water, ethyl alcohol and a mixture of glycerol and water (the percentage is not stated). Depew and August looked to improve on previously proposed correlations and supplied the following equation (Depew and August, 1971).

$$
\mathrm{Nu}\left(\frac{\mu_{\mathrm{w}}}{\mu_{B}}\right)^{0.14}=1.75\left(\mathrm{Gz}+0.12\left(\mathrm{GzGr}^{1 / 3} \mathrm{Pr}^{0.36}\right)^{0.88}\right)^{\frac{1}{3}}
$$

\subsection{Tabulated Summary of Literature}

Table 2-1 summarises the Nusselt number correlations for the literature cases found in this chapter. The information given is that found in the papers themselves, and therefore not all parameters are available for all cases.

Table 2-1: Summary of Nusselt number correlations

\section{Yasuo, et al. (1966)}

Uniform Heat Flux

Medium: Air

Horizontal Tubes

$100<\operatorname{Re}<13000$

$$
\mathrm{Nu}=0.61(\mathrm{ReRa})^{\frac{1}{5}}\left[1+\frac{1.8}{(\mathrm{ReRa})^{\frac{1}{5}}}\right]
$$

\section{Kupper, et al. (1969)}

Uniform Heat Flux

Medium: Water

Horizontal Tube

$100<\operatorname{Re}<2000,300<\mathrm{Gr}<30000,4<\operatorname{Pr}<9$ 


$$
\mathrm{Nu}=\frac{48}{11}+0.048 \operatorname{Pr}^{\frac{1}{3}}(\operatorname{ReRa})^{\frac{1}{5}}
$$

\section{Morcos and Bergles (1975)}

Uniform Heat Flux

Medium: Distilled Water \& Ethylene Glycol

Horizontal Tube

$3 \times 10^{4}<\mathrm{Ra}<10^{6}, 2<P w^{*}<66,4<\mathrm{Pr}<175$

$$
\mathrm{Nu}=\left\{(4.36)^{2}+\left[0.145\left(\frac{\mathrm{Gr}^{*} \operatorname{Pr}^{* 1.35}}{P w^{* 0.25}}\right)^{0.265}\right]^{2}\right\}^{1 / 2}
$$

\section{Ghajar and Tam (1994)}

Uniform Heat Flux

Medium: Water \& Ethylene Glycol

Horizontal Tube

$280<\operatorname{Re}<3800$

$$
\mathrm{Nu}=1.24\left((\operatorname{RePr} D / L)+0.025(\mathrm{GrPr})^{0.75}\right)^{\frac{1}{3}}\left(\mu / \mu_{w}\right)^{0.14}
$$

\section{Mohammed and Salman (2007)}

Uniform Heat Flux

Medium: Air

Horizontal Tube

$400<\operatorname{Re}<1600$

$$
\overline{\mathrm{Nu}}=3.19(\overline{\mathrm{Ra}} / \overline{\mathrm{Re}})^{0.26}
$$

\section{Oliver (1962)}

Uniform Temperature

Medium: Water, ethyl alcohol and glycerol in water

Horizontal Tube

$29<\mathrm{Gr}<1.6 \times 10^{5}, 1.9<\operatorname{Pr}<326$

$$
\mathrm{Nu}\left(\frac{\mu_{\mathrm{w}}}{\mu_{B}}\right)^{0.14}=1.75\left(\mathrm{Gz}+5.6 \times 10^{-4}\left(\operatorname{GrPr}\left(\frac{L}{D}\right)\right)^{0.7}\right)^{\frac{1}{3}}
$$

\section{Brown and Thomas (1965)}

Uniform Temperature

Medium: Water

Horizontal Tube

$$
\mathrm{Nu}=1.75\left(\frac{\mu_{\mathrm{B}}}{\mu_{w}}\right)^{0.14}\left(\mathrm{Gz}+0.012\left(\mathrm{GzGr}^{\frac{1}{3}}\right)^{4 / 3}\right)^{\frac{1}{3}}
$$

\section{Depew and August (1971)}

Uniform Temperature

Medium: Water, ethyl alcohol and glycerol in water

Horizontal Tube

$\operatorname{Re}<1800$

$$
\mathrm{Nu}\left(\frac{\mu_{\mathrm{w}}}{\mu_{B}}\right)^{0.14}=1.75\left(\mathrm{Gz}+0.12\left(\mathrm{GzGr}^{1 / 3} \operatorname{Pr}^{0.36}\right)^{0.88}\right)^{\frac{1}{3}}
$$




\subsection{Summary of Chapter}

This chapter gave an overview of previous works done in the fields of uniform and non-uniform heat flux boundary conditions. A large amount of the work that was reviewed investigated the production and consequences of buoyancy driven secondary flow. It was quickly noted that secondary flow plays an integral part of heat transfer problems and cannot simply be ignored. The heat transfer coefficient and Nusselt number can be enhanced by up to three or four times when comparing them to traditional constant property solutions when secondary flow is present. It was also noted that secondary flow requires an entry length before it can fully develop. Since there is a lack of previous works focused on non-uniform heat flux boundary conditions, the work reviewed was not as focused as seen for the previous works on the uniform heat flux cases. The literature becomes more directed towards the current work the later in the chapter with the final numerical case (Okafor et al., 2014) coming very close to the experimental case discussed in this dissertation. It was seen that the heat transfer coefficient is larger when comparing cases with non-uniform heat fluxes to those with uniform heat flux boundary conditions. This shows that the non-uniformity of the heat flux does have an influence on the heat transfer and non-uniform heat flux cases cannot be treated in the same manner as uniform heat flux cases.

In the next chapter the experimental test facility that was used in this investigation is described. 


\section{Experimental Setup}

\subsection{Introduction}

This chapter describes the experimental setup that was used in this study. As mentioned, it was required to apply different circumferential heat flux profiles to a circular tube and to obtain experimental data (fluid temperature, wall temperature and heat fluxes) to determine the local and overall heat transfer coefficients at different mass flow rates. In section 3.1 the laboratory test facility is described, while in section 3.2 the tube test section is described.

\subsection{Experimental Facility}

Figure 3-1 gives a representation of the test facility that was used and which was housed in the thermo-flow research laboratory at the University of Pretoria. It consisted of a closed water loop which supplied water at preselected temperatures to an electrically heated test section. Temperature stability was maintained via the use of a $1000 \mathrm{~L}$ storage tank which was thermostatically controlled and connected to a $45 \mathrm{~kW}$ chiller unit (item 1). Depending on the test requirements, water was passed at preselected mass flow rates through the test line (which contained the test section) and the bypass line by means of a positive displacement pump (item 2). Because many of the test case flow conditions were for mass flow rates that were significantly lower than the rated mass flow rate of the pump, the bypass line was useful to ensure that stable flow rates through the test line could be achieved by adjusting the hand-operated ball valve (item $7 a$ ), the other hand-operated ball valves (items $7 \mathrm{~b}$ and 7c) were left completely open during testing and were installed for safety purposes. An accumulator (item 3) was used to further reduce flow pulsations, while a filter (item 4) was used to prevent small particles from entering the test line which might affect the results. Pressure gauges (items $5 a-c$ ) were installed to ensure that the pressure in the system could be monitored (pressure readings were not logged since they were not needed during data reduction). A Coriolis mass flow meter (item 6) with a measuring range of 0 to $0.604 \mathrm{~kg} / \mathrm{s}$ and an accuracy of $0.05 \%$ of the full range was used to determine the water flow rate through the test line. A non-return valve (item 8) was used to isolate sections of the water loop as required. The electrically heated test section (item 9) was connected to a $3 \mathrm{~kW} \mathrm{DC}$ power supply (item 10), with voltages and electric current ranges of 0 to $360 \mathrm{~V}$ and 0 to $30 \mathrm{~A}$ respectively. The accuracy of the power supply was $0.15 \%$ for both the voltage and current. Temperature probes on the test section (which will be described in more detail in Section 3.3) and the mass flow meters were connected to a National Instruments SCXI 130332 terminal block fitted to a National Instruments chassis and a desktop computer (item 11). National Instruments LabVIEW software and MATLAB script were used for data acquisition purposes. 


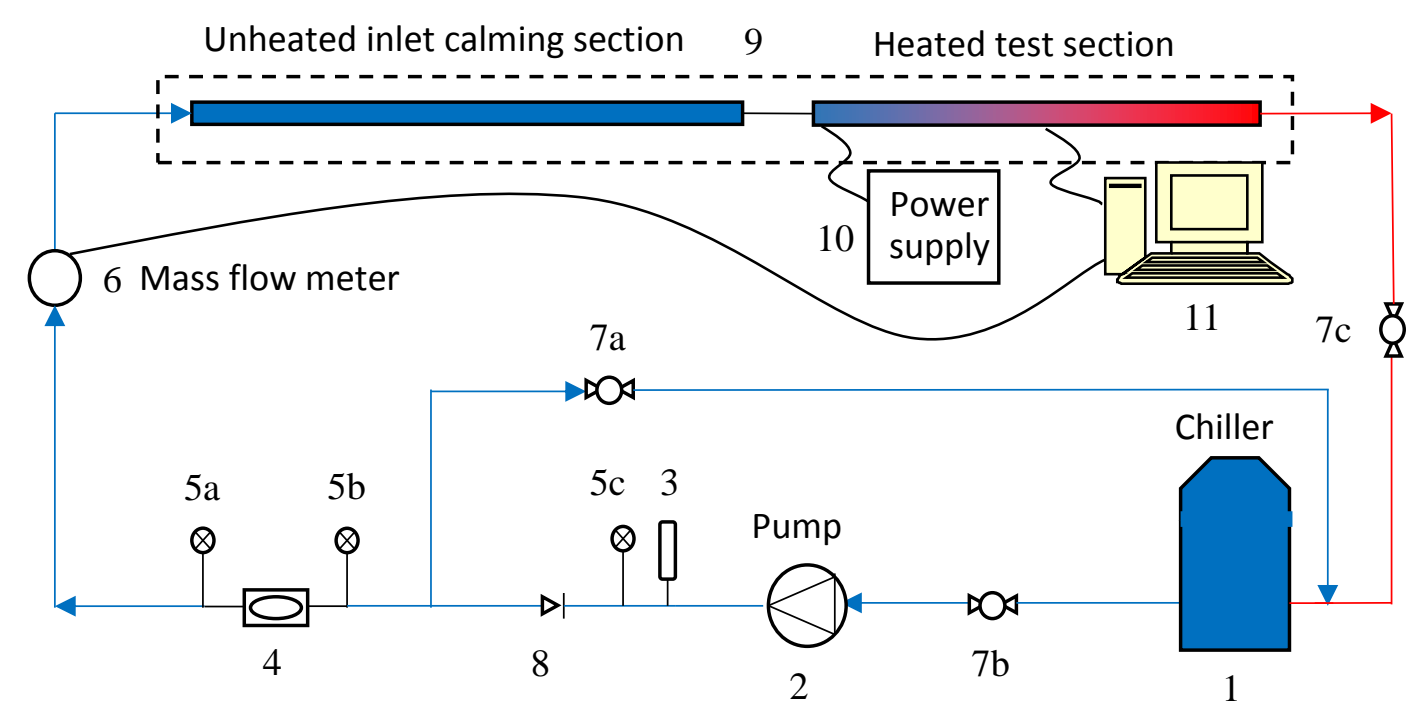

Figure 3-1: Schematic of experimental setup

\subsection{Test Section}

Figure 3-2 shows a schematic side view of the test section. It consisted of a bulk fluid inlet temperature measuring station at $m=0$, an unheated (isothermal) inlet calming section, an electric heated section with wall temperature measurement stations, and a bulk fluid outlet temperature measuring station at $m=8$.

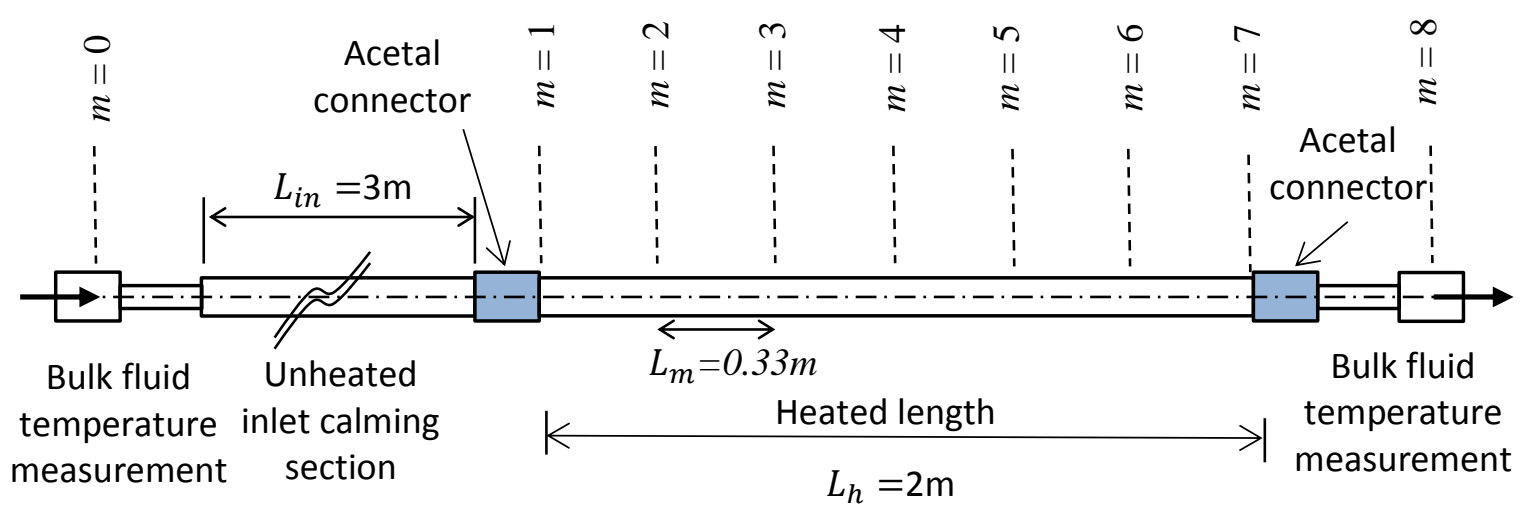

Figure 3-2: Side view schematic of the test section (not drawn to scale)

\subsubsection{Bulk Fluid Inlet and Outlet Measuring Stations}

In order to determine bulk fluid properties and to calculate the effective energy transfer rate to the water in the test section, the inlet and outlet bulk fluid temperatures of the water were measured at specially designed measuring stations at $m=0$ and $m=8$. Each measurement station consisted of a copper tube having a length of $130 \mathrm{~mm}$, an inner diameter of $18 \mathrm{~mm}$ and an outer diameter of $20 \mathrm{~mm}$. On each of these, four T-type thermocouples were equally spaced around the circumference and soldered onto the outside on the downstream end. During steady state conditions the copper reached 
thermal equilibrium with the internal water. This allowed for the determination of the internal fluid temperature by measuring the outside temperature of the copper.

However, because most of the test conditions in this investigation were in the laminar flow regime, care had to be taken to disturb any thermal layering in the fluid, especially at the outlet measuring station. This was done by making use of internal flow mixers which consisted of twisted copper strips as is represented in Figure 3-3, and which were located in the upstream portions of both of the measuring stations. Further improvement in the measurement accuracy of the inlet and outlet temperatures was obtained by making use of the arithmetic average of the readings of the four thermocouples at each station. This reduced the temperature measurement uncertainty as compared to when only a single thermocouple at each station would have been used. More information on this and other measurement uncertainties is supplied in a later section of this dissertation.

To prevent axial heat conduction to or from the measurement locations, each copper section was connected in-line with the rest of the test section and the flow loop circuit by making use of flexible hoses (having a thermal conductivity of $0.19 \mathrm{~W} / \mathrm{mK}$ ). Thermal insulation from the lab was ensured by covering each station with Armaflex type thermal insulation which had a thickness of $100 \mathrm{~mm}$ and a thermal conductivity of $k_{\text {insul }}=0.036 \mathrm{~W} / \mathrm{mK}$.

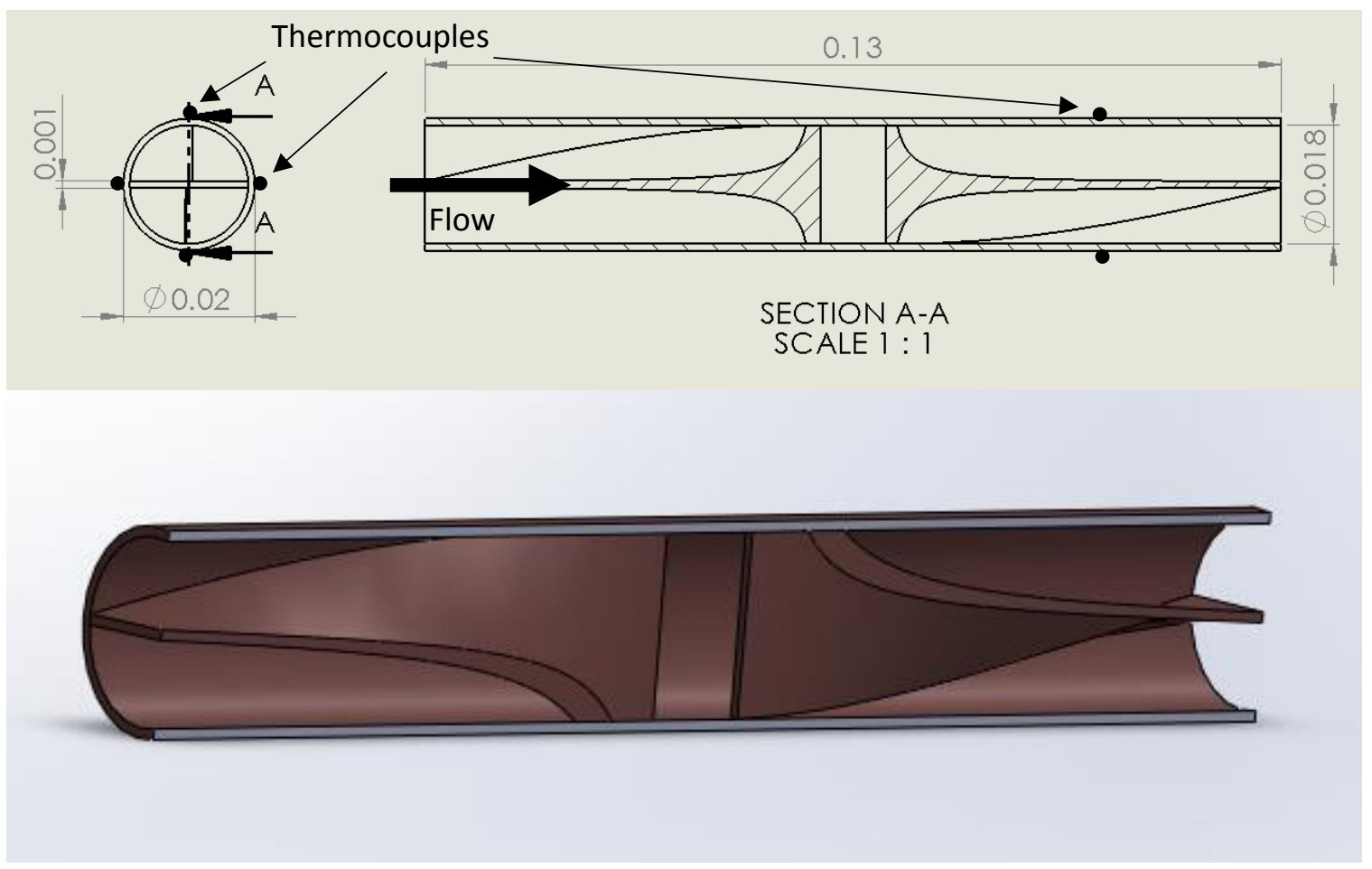

Figure 3-3: Mixer dimensions and section view

\subsubsection{Unheated Inlet Calming Section}

After the inlet bulk fluid temperature measurement station, the incoming flow was conditioned and calmed by making use of an isothermal (adiabatic) inlet tube (stainless steel 304). The tube was premanufactured via extrusion to produce a seamless tube. It had the same inner diameter $(D=27.8 \mathrm{~mm})$, wall thickness $(t=2.77 \mathrm{~mm})$ and outer diameter $\left(D_{o}=33.34 \mathrm{~mm}\right)$ as the heated portion of the test section. Its length $\left(L_{i n}=3 \mathrm{~m}>100 D\right)$ was selected to provide hydrodynamically developed flow at the start of the heated portion. This was important because the inlet flow velocity distribution 
profile can have a significant impact on the thermal behaviour in laminar and transitional flow regimes (Dirker et al., 2014).

The inlet tube section was thermally separated (insulated) from the heated section by a carefully machined Acetal bush as is shown in Figure 3-4. Acetal was selected because it has a low thermal conductivity $(k=0.5 \mathrm{~W} / \mathrm{mK})$ and it is easy to machine. The bush had an axial length of $130 \mathrm{~mm}$ and the same internal diameter as the inlet tube and the heated tube portions. The bush was carefully attached to the tube sections via compression fits. Rubber hoses were secured over either end of the bush to prevent leaks. Care was taken to ensure a smooth internal surface at these joints by enforcing strict manufacturing tolerances on the bush to reduce undesired flow disturbances. The inlet length was insulated from the laboratory by a layer of Armaflex insulation $130 \mathrm{~mm}$ thick, with a thermal conductivity of $0.036 \mathrm{~W} / \mathrm{mK}$.

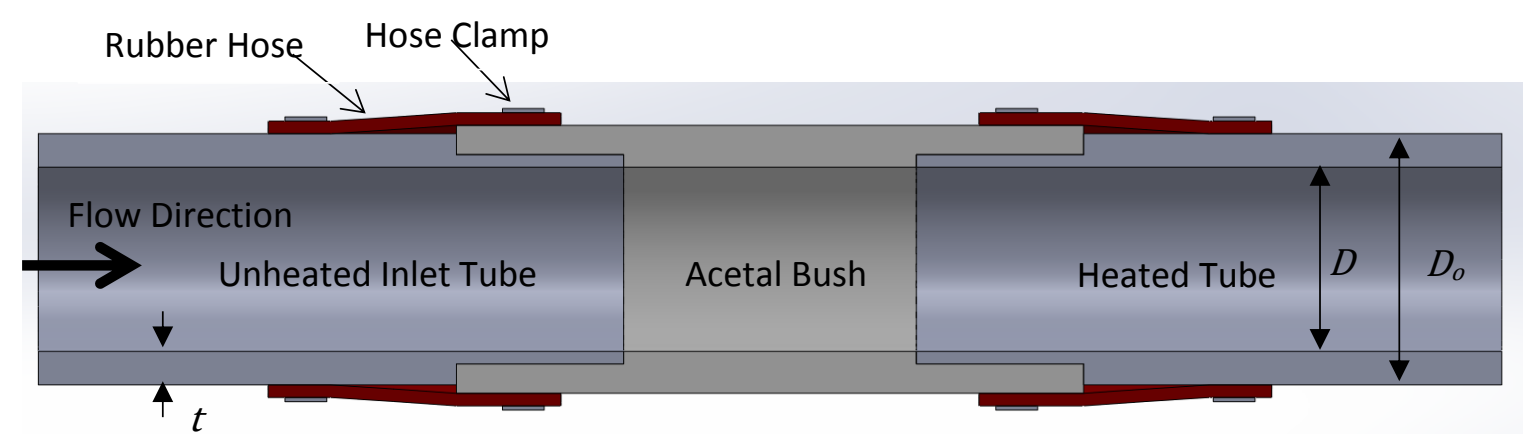

Figure 3-4: Cross section of the connection between the unheated inlet tube and the heated tube

\subsubsection{Heated Test Section}

The heated tube section, which was also made of same type of stainless steel tube as the unheated inlet section (stainless steel 304), had a length of $2.00 \mathrm{~m}\left(L_{h}\right)$ and was designed to facilitate different heat flux boundary conditions and to enable the measurement of the associated wall temperature profiles. Its specialized design is given schematically in Figure 3-5 (a) the cross sectional view down the length of the tube in the direction of the flow, and (b) a side-view section perpendicular to the flow.

(a)

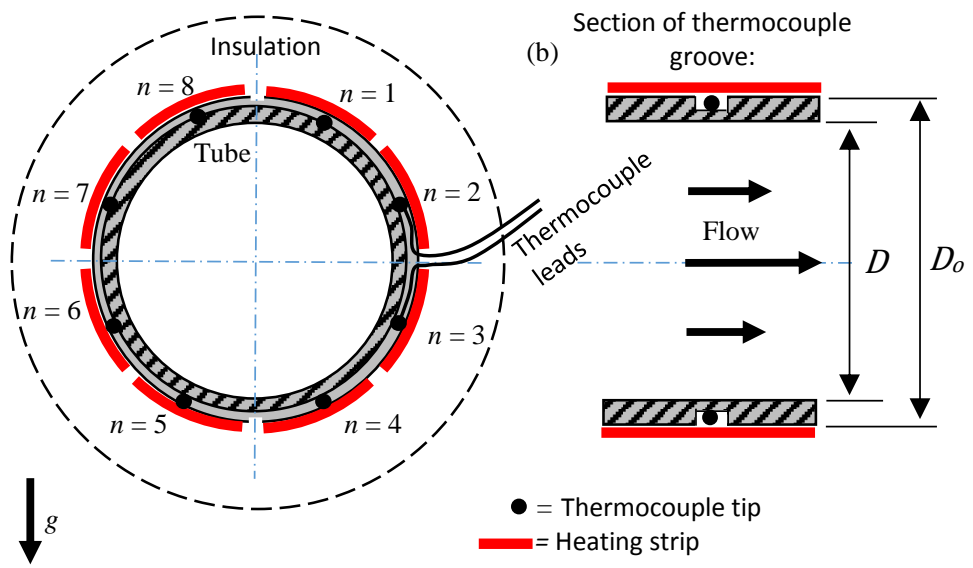

Figure 3-5: Detailed view of the thermocouple tip and heating element placement (not to scale) 
In order to produce different circumferential heat flux conditions, the tube was equipped with 8 heating element strips which were equally spaced around the circumference of the tube and which ran the entire length of the heated portion of the test section. The heating elements are shown in red in Figure 3-5 and are numbered from $n=1$ to $n=8$. Each heating element consisted of a four-pass constantan heating wire (Figure 3-6 a) which was directly attached to the outside wall of the tube by means of heat resistant tape to ensure good thermal contact with the tube, as seen in Figure 3-6 b). The constantan wire had a diameter of $0.38 \mathrm{~mm}$ and had extruded PFA electrical insulation to prevent an electric short circuit to the stainless steel pipe. The element strips, individually, had an average resistance of $106.2 \Omega$, with a maximum variance from the average of $2.2 \Omega$. This means that all the elements had electrical resistances within $2 \%$ of the average resistance. When installed, each element covered a $45^{\circ}$ angle span of the outer surface of the tube and resulted in a $1.8 \mathrm{~mm}$ tangential gap between each adjacent heating element strip.

a)

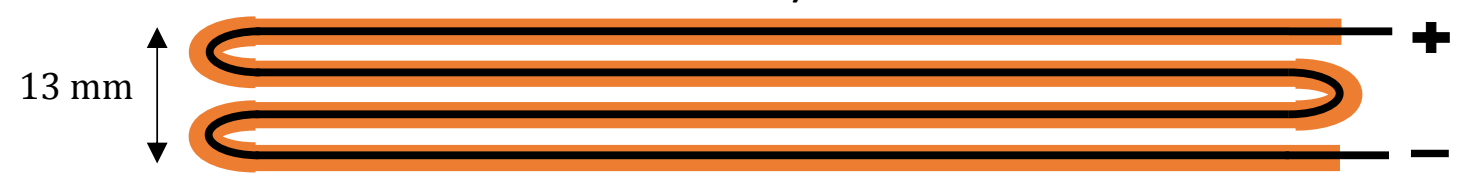

b)

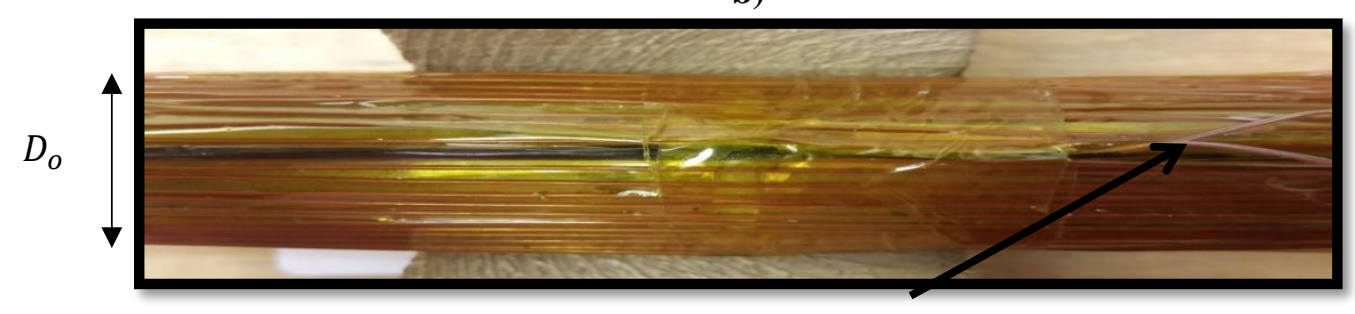

Thermocouple Leads

Figure 3-6: a) Schematic of a single four-pass heating element strip. b) Photograph of the heating element strips attached to the pipe wall with heat resistant tape, as well as the thermocouple leads leaving the heated section between the heating element strips.

Each heating element could be powered individually which enabled the investigation of various heat flux boundary distributions. For instance, when all eight elements were powered, an approximately uniform heat flux boundary was imposed onto the tube, while when elements $n=3$ to $n=6$ were powered, only the bottom half of the tube was heated. Various other heat flux boundary conditions were investigated in this study as will be described later in more detail. For this purpose, each element was controlled by a switch and connected to the DC power supply that was mentioned earlier. Figure 3-7 shows the circuit diagram for the heating element connections to the DC power supply. The parallel configuration allowed for each of the elements to be individually controlled and also ensured that all the elements were energised with the same electrical voltage potential.

Because the main focus of this study was the investigation of the effects of the non-uniform heat flux boundary condition, the boundary conditions applied on the wetted surface needed to be unequivocally defined. As mentioned, stainless steel was selected as the pipe material. This was partly done due to its low thermal conductivity $(k=16 \mathrm{~W} / \mathrm{mK})$ which resulted in relatively low levels of heat spreading around the circumference of the tube, especially when only a select few of the heating 
elements were energized. This meant that non-uniform heat flux boundary distributions on the outside of the tube were relatively well transferred to the wetted inner surface of the tube. High levels of tangential conduction of heat around the circumference of the pipe would cause the boundary conditions of the experiment to become less defined and resulted in the dissipation of heat away from the intended heating positions. The low thermal conductivity was also important to reduce the amount of heat conducted axially along the pipe which was calculated to be less than $1 \%$ of the heat transferred to the water in this study, and was seen as negligible.

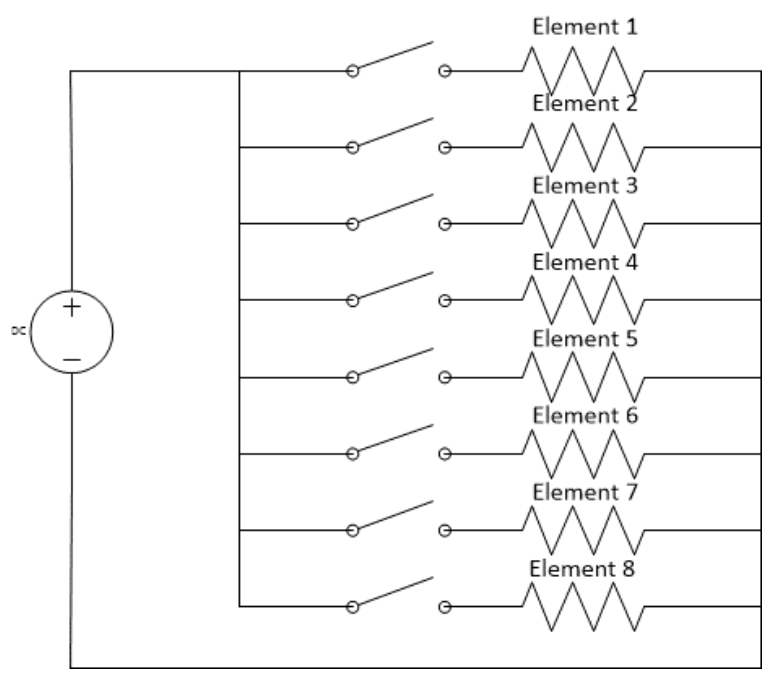

Figure 3-7: Circuit diagram of element connection to DC power supply

The heated tube was also divided into six axial sections, each with an axial length of $L=333 \mathrm{~mm}$ as is seen in Figure 3-2. This resulted in the definition of positions $m=1$ to $m=7$ which were very useful during the data analysis procedure as will be described later. At locations $m=2$ to $m=6$, wall temperature measuring stations were constructed by machining a groove at each location around the circumference of the tube. Each groove housed $8 \mathrm{~T}$-type thermocouples $(0.5 \mathrm{~mm}$ in diameter) as is shown in Figure 3-5. Thus, 40 thermocouples were used to obtain the temperature profile around and along the heated tube. Each groove was $1.77 \mathrm{~mm}$ deep and $3.2 \mathrm{~mm}$ wide. This meant that a wall thickness of approximately $1 \mathrm{~mm}$ was left between the inside wall of the tube and the bottom of each groove.

The thermocouples were positioned directly below the heating elements and were fully contained in the grooves as is shown in Figure 3-5 and Figure 3-8. Each tip was held in position by means of Electrolube epoxy thermal adhesive with a thermal conductivity of $1.1 \mathrm{~W} / \mathrm{mK}$. Before adhesion, care was taken to clean the grooves with acetone to remove any grease. The installation of the thermocouple tips was done with emphasis on precision to ensure that each thermocouple tip was in contact with the pipes surface. This ensured that the thermocouples captured the stainless steel wall temperatures and not the heating element temperatures. Each installed thermocouple tip was carefully photographed in order to identify its installed position for later use during calibration and data reduction. The thermocouple leads were kept in the groove for a few millimeters before being fed out between the heating element strips as can be seen in Figure 3-5 and Figure 3-8. Axial heat conduction in the thermocouple leads was checked and found to introduce a maximum error of approximately $0.3^{\circ} \mathrm{C}$. 
Once the heating element strips were securely fastened, and the thermocouple leads had been moved clear of the heating elements, the tube was tightly wrapped with an insulating rubber tape. Hose clamps were also closed around the pipe along its length at intervals of $10 \mathrm{~mm}$. This was done to ensure that the heating element strips were securely held in contact with the tube along the entire length and that the thermal contact resistance was relatively uniform along and around the pipe. Failure to maintain uniform thermal resistance between the heating elements and the tube would have resulted in localized hotspots which would have placed the accuracy of measurement in jeopardy.

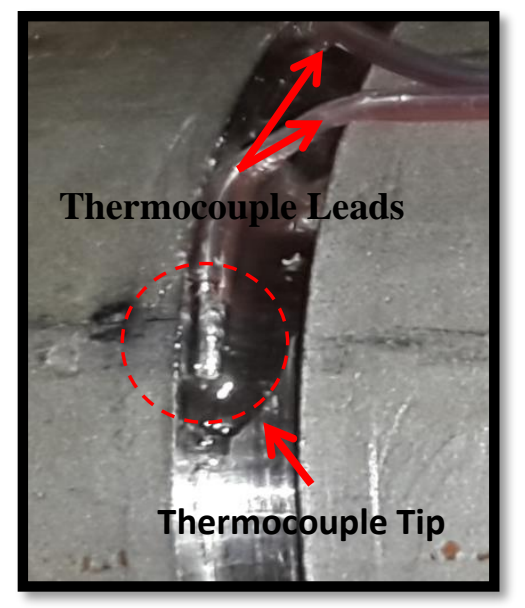

Figure 3-8: Thermocouple tip and lead placement

As with the isothermal inlet length, a layer of Armaflex insulation $130 \mathrm{~mm}$ thick, with a thermal conductivity of $0.036 \mathrm{~W} / \mathrm{mK}$ was used to insulate the heated tube from the laboratory. The thickness of the insulation resulted in an anticipated maximum heat loss of $3 \%$ of the total heat input based on worst case scenario ambient laboratory temperatures.

\subsection{Summary of Chapter}

This chapter presented the experimental setup to the reader. The system was designed with special thought to keep the uncertainties to a minimum and to promote repeatability. Preselected nonuniform heating boundary conditions could be investigated by making use of a low thermal conductivity pipe equipped with 40 wall temperature thermocouples, and 8 heating elements which were equally spaced around the circumference of the heated portion. This allowed for various different heat flux distributions to be investigated. In the next chapter the experimental procedure is described. 


\section{Experimental Procedure}

\subsection{Introduction}

In this chapter an overview of the experimental procedure is given. Before any tests were performed, all measuring devices and probes were calibrated and the test facility was commissioned. This is discussed first. There-after the experimental method and the test matrix is presented.

\subsection{Thermocouple Calibration}

After the thermocouples were installed onto the relevant positions in the test section, they were calibrated in situ by operating the test bench at a series of isothermal tests (adiabatic) at different temperature states. Other calibration techniques also exist, such as the use of a thermal bath, but were deemed unsuitable or difficult to implement in this study because of the way the thermocouples were connected to the test section. This is particularly of importance for the thermocouples that were soldered to the inlet and outlet bulk fluid temperature measurement stations ( $m=0$ and $m=8$ ). If pre-calibrated thermocouples were used, the heating of the solder during the attachment procedure would have altered the thermocouple tips, as well as any calibrated thermal response behaviour of the thermocouples. In contrast, the in situ method allowed for all the thermocouples to be calibrated at the same time and under the same conditions in their installed conditions.

Each in situ calibration run was performed by connecting the system to a thermostatically controlled electric heated tank and circulating water through the test section at a mass flow rate of $0.2 \mathrm{~kg} / \mathrm{s}$ which represented highly turbulent flow. This caused sufficient internal mixing which ensured uniform water temperature throughout the system. Two calibrated (to an accuracy of $0.1^{\circ} \mathrm{C}$ ) PT100 probes were connected (and well insulated) at either end of the test section, before and after the measuring stations at $m=0$ and $m=8$ respectively.

The calibrations were done at water temperatures ranging approximately from $20^{\circ} \mathrm{C}$ to $53^{\circ} \mathrm{C}$ using increments of about $5^{\circ} \mathrm{C}$. For each temperature condition, data was recorded once a suitable steady state condition had been met. The steady state condition chosen for the calibration phase of this study was when the PT100 probe readings remained unchanged for two minutes within a margin of $0.1^{\circ} \mathrm{C}$.

Once this data was gathered, calibration curves were drawn as can be seen in Figure 4-1 from which calibration factors were found. Figure 4-1 shows the difference between the original data points and the readings from the PT100 probe sensors (already calibrated), as well as the difference between the calibrated data points and the temperature readings of the PT100 sensor for a specific thermocouple (TC4). Figure 4-1 gives a good indication as to how the thermocouple readings were calibrated to ensure more accurate results. The calibration second order polynomial results can be found in Appendix B. Calibration factors were subsequently applied to all measured temperature data before the data was analyzed. Based on the calibration results it was found that the thermocouples were all calibrated to within $0.13^{\circ} \mathrm{C}$. 


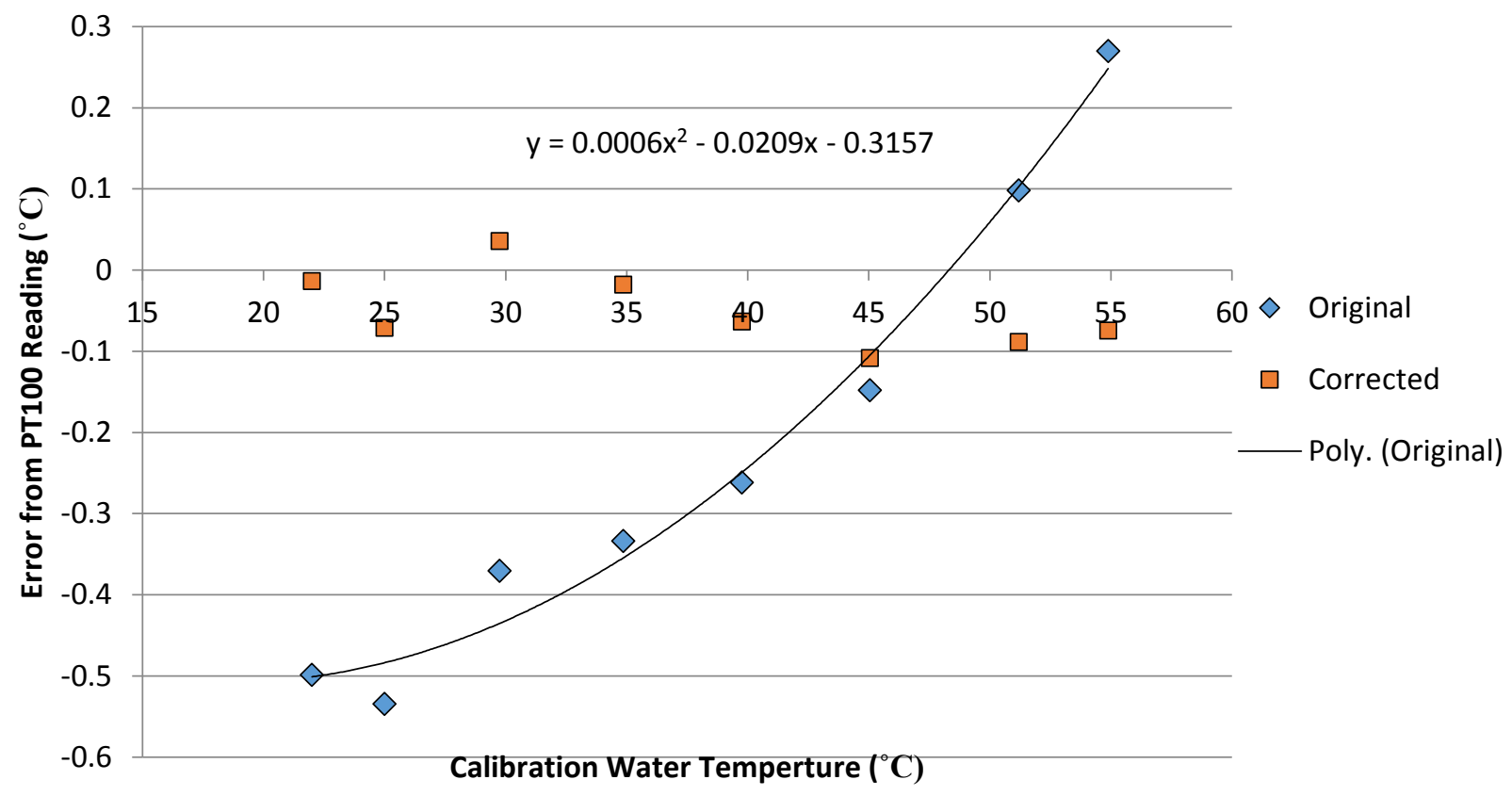

Figure 4-1: Example of calibration curve for $m=2$, TC4

\subsection{Test Procedure and Test Matrix}

Table 4-1 and Figure 4-2 show the different test configurations considered in this study. In total 16 case types were considered. The table contains the case number, the heated span, the heating elements that were switched on, the total power entering the system and the associated local heat flux at the heated arc. Five heat flux intensities appear in the table. For convenience, highlight coloring is used to indicate which test cases shared the same heat flux. Based on the heated spans and the total energy rate supplied to the heating elements, local heat flux values ranging from $1658 \mathrm{~W} / \mathrm{m}^{2}$ to $6631 \mathrm{~W} / \mathrm{m}^{2}$ were achieved. Due to the laboratory limitations, the experimentally applied heat flux could not be increased to match commercial heat flux ranges, but as will be shown later, the selection of the heat fluxes in this study is suitable to determine the influence of heating position on the effective heat transfer coefficient.

Table 4-1: Test cases with heating degrees and corresponding active heating elements

\begin{tabular}{|c|c|c|c|c|}
\hline $\begin{array}{c}\text { Case } \\
\text { number }\end{array}$ & Heated span & Powered heating strips $(n)$ & $\dot{Q}_{\text {tot }}[\mathrm{W}]$ & $\dot{q}\left[\mathrm{~W} / \mathrm{m}^{2}\right]$ \\
\hline $\mathbf{1}$ & $360^{\circ}$ & $1,2,3,4,5,6,7,8$ & 800 & 4421 \\
\hline $\mathbf{2}$ & $360^{\circ}$ & $1,2,3,4,5,6,7,8$ & 600 & 3316 \\
\hline $\mathbf{3}$ & $360^{\circ}$ & $1,2,3,4,5,6,7,8$ & 400 & 2210 \\
\hline $\mathbf{4}$ & $360^{\circ}$ & $1,2,3,4,5,6,7,8$ & 300 & 1658 \\
\hline $\mathbf{5}$ & Lower $180^{\circ}$ & $3,4,5,6$ & 600 & 6631 \\
\hline $\mathbf{6}$ & Lower $180^{\circ}$ & $3,4,5,6$ & 400 & 4421 \\
\hline $\mathbf{7}$ & Lower $180^{\circ}$ & $3,4,5,6$ & 300 & 3316 \\
\hline $\mathbf{8}$ & Upper $180^{\circ}$ & $1,2,7,8$ & 600 & 6631 \\
\hline $\mathbf{9}$ & Upper $180^{\circ}$ & $1,2,7,8$ & 400 & 4421 \\
\hline $\mathbf{1 0}$ & Upper $180^{\circ}$ & $1,2,7,8$ & 300 & 3316 \\
\hline $\mathbf{1 1}$ & Lower $90^{\circ}$ & 4,5 & 300 & 6631 \\
\hline $\mathbf{1 2}$ & Upper $90^{\circ}$ & 1,8 & 300 & 6631 \\
\hline
\end{tabular}




\begin{tabular}{|l|c|c|c|c|}
\hline $\mathbf{1 3}$ & Right $180^{\circ}$ & $1,2,3,4$ & 600 & 6631 \\
\hline $\mathbf{1 4}$ & Left $180^{\circ}$ & $5,6,7,8$ & 600 & 6631 \\
\hline $\mathbf{1 5}$ & Slanted $180^{\circ}$ & $2,3,4,5$ & 600 & 6631 \\
\hline $\mathbf{1 6}$ & Slanted $180^{\circ}$ & $2,3,4,5$ & 400 & 4421 \\
\hline
\end{tabular}

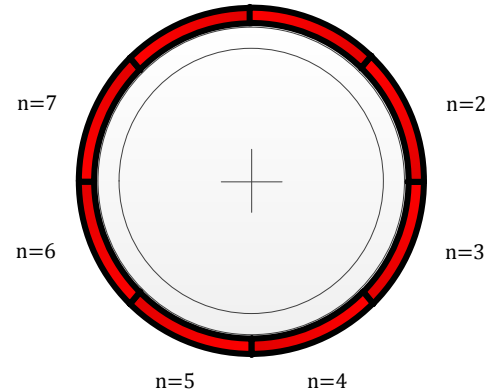

Cases $1,2,3,4$

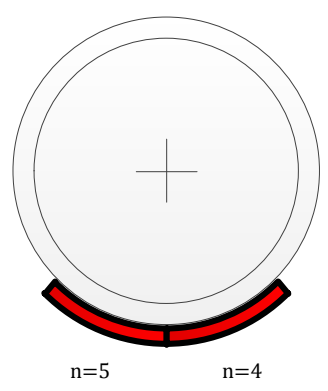

Cases 11

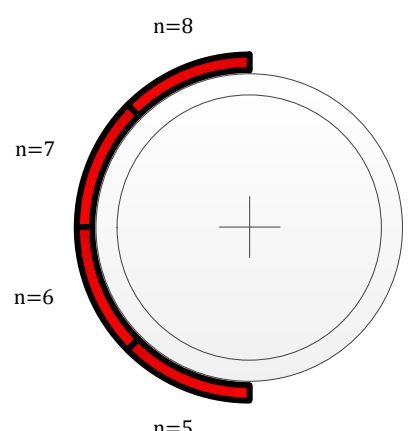

Cases 14

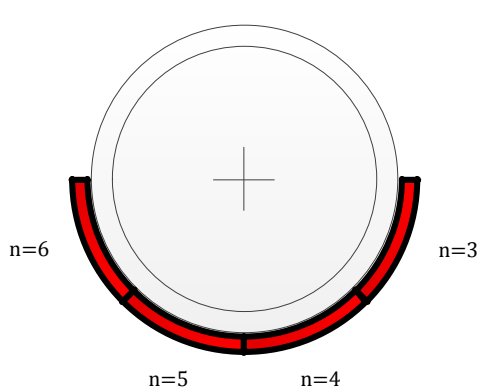

Cases $5,6,7$

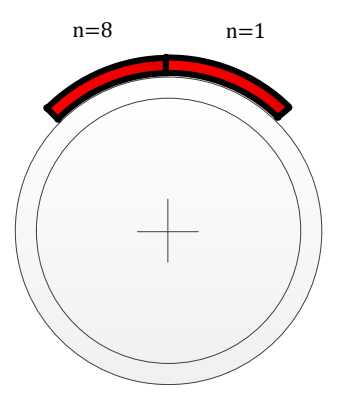

Cases 12

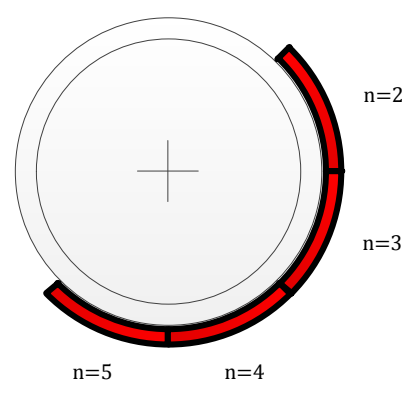

Cases 15,16

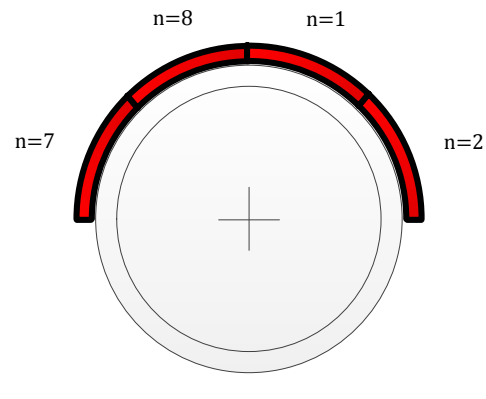

Cases $8,9,10$

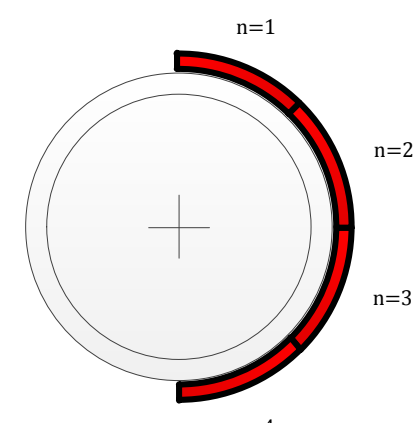

Cases 13

Powered Heating Element

Figure 4-2: Graphical representation of test cases

The test cases were chosen carefully. The first four cases were for $360^{\circ} /$ uniform heating, with four different applied heat fluxes. These cases were used as a reference to identify the difference between uniform and non-uniform heating cases and also to identify what impact the heat flux has. The difference between lower and upper heating at an angle span of $180^{\circ}$ is considered in cases 5,6 and 7 , and cases 8,9 and 10 respectively at three different heat fluxes. These tests allowed for the results to be compared to the uniform heating cases as well as to be compared to each other in order to observe the effect of heating position on buoyancy secondary flow. In cases 11 and 12 smaller angle spans of lower and upper $90^{\circ}$ span heating cases were considered at a single heat flux. This allowed for the observation of the influence of the angle span at the same heat flux (by comparing it with cases 5 and 8 ) as well as at the same total heat input (by comparing it with cases 7 and 10). Lateral side heating was considered in case 13 and 14 with an angle span of $180^{\circ}$. This allowed for checking 
symmetric behaviour of the test section and to better identify the influence of direction of gravity by comparing these cases with the other $180^{\circ}$ cases. Lastly, cases 15 and 16 , which had slanted heat flux positions, were used to further identify the impact of the gravitational direction at two heat flux levels. The slanted heat flux position is representative of non-noon (morning or afternoon) conditions in a linear parabolic trough or Fresnel solar collector.

Before each experimental test run, water was circulated through the system until the chiller was able to provide water from the reservoir at a constant inlet temperature to the test section of approximately $20^{\circ} \mathrm{C}$. The desired heat intensity was set by selecting the voltage output on the DC power supply and the heating elements were then switched on according to the desired case in Table 4-1. The pump was adjusted in order to bring the mass flow of the water to the highest Reynolds number which would be tested. As will be shown later, an upper bound Reynolds number of 2600 was selected for most of the test cases to ensure that the tests fell in the laminar flow regime and not partially in the transitional flow regime. The pump was left on this setting until a suitable steady state condition had been met. Data was logged once steady state conditions were reached. Steady state for this phase of the investigation was defined as when the value for the temperature difference $(\Delta T)$ between the arithmetic average inlet $(m=0)$ and outlet $(m=8)$ temperatures did not fluctuate by more than $0.1^{\circ} \mathrm{C}$ for one minute.

$$
\Delta T=\bar{T}_{\text {in }}-\bar{T}_{\text {out }}
$$

The energy balance was also used as part of the steady state condition check. When the temperature difference aspect of the steady state condition was met, a first order estimate of the energy balance was calculated. Data was only logged if it was below $5 \%$. The first order estimate did not yet account for known heat losses such as through the insulation (of about $3 \%$ of the total heat transfer rate), and will be discussed in more detail in the next chapter. Once these criteria were met, a minimum of 200 data points were captured and logged at a frequency of $20 \mathrm{~Hz}$. Once the data points for the first Reynolds number $(\operatorname{Re}=2600$ ) were captured, the Reynolds number was slowly decreased by roughly 150 for each subsequent case until the lowest Reynolds number case of approximately $\operatorname{Re}=650$ was reached. The reason for performing the tests in a sequence of decreasing Reynolds number was because the systems temperature increased as the Reynolds number was decreased. If the low Reynolds numbers were tested first there was a risk of the insulation absorbing too much heat and becoming a heat source itself.

\subsection{Summary of Chapter}

The calibration of the thermocouples is contained in this chapter. The different test cases and a graphical representation of each case are also contained here. This chapter serves to show that the utmost care and thought was put into the planning of the experimental process in order to get the most accurate results possible. In the next chapter the data reduction technique is described once experimental data was obtained. 


\section{Data Reduction}

\subsection{Introduction}

This chapter contains the methods used to reduce the raw experimental data of the thermocouples, mass flow meter and energy inputs into meaningful results. The data reduction includes those methods used to find the energy balances, the tangential heat transfer in the pipe, as well as the Nusselt numbers and heat transfer coefficients. An overview of uncertainty and sensitivity analyses is also included.

As mentioned, 200 data points were logged at $20 \mathrm{~Hz}$ for each measurement device. This resulted in a sample of data taken of a period of 10 seconds. These 200 data points were then averaged to obtain a single data point for each measurement device.

\subsection{Energy Balance}

The energy balance error was an important quantity to quantify for each test condition. For this purpose all incoming and outgoing energy rates to or from the test section were required.

The power input from the power supply, $\dot{Q}_{i n}$, was calculated using:

$$
\dot{Q}_{\text {in }, \text { tot }}=I_{t o t} V_{D C}
$$

The total power input was then divided between the heating elements that were switched on (due to their parallel connection). The maximum deviation of the average electrical resistance of $2 \%$ and the calibration of the heating elements was enough to ensure that the total input power could simply be divided into the switched on heating elements using the parallel switch system. The voltage $\left(V_{D C}\right)$ and current $\left(I_{\text {tot }}\right)$ were directly logged from the power supply. The amount of heat transferred to the water itself was calculated using:

$$
\dot{Q}_{\text {out }}=\dot{m} C_{p}\left(\bar{T}_{\text {out }}-\bar{T}_{\text {in }}\right)
$$

Here $\dot{m}$ was obtained from the mass flow meter and $\bar{T}_{\text {out }}$ and $\bar{T}_{\text {in }}$ were determined by taking the arithmetic average of the thermocouples at measuring stations $m=0$ and $m=8$ respectively. The specific heat value, $C_{p}$, was evaluated (using equations put forward by Popiel and Wojtkowiak (1998)) at the average bulk temperature within the test section. To ensure the accuracy of the results and that the selected thickness of insulation was sufficient an energy balance was performed before continuing with the data reduction process. The first order energy balance error (\% value) was calculated as follows:

$$
E B=100 \frac{\left|\dot{Q}_{\text {in }}-\dot{Q}_{\text {out }}\right|}{\frac{1}{2}\left(\left|\dot{Q}_{\text {in }}\right|+\left|\dot{Q}_{\text {out }}\right|\right)}
$$

In this study the maximum energy balance error was $5 \%$ while $85 \%$ of all the data had an error of less than $3 \%$. For calculation purposes, heat rates were based on the water side heat transfer since this had a lower measuring uncertainty. The heat loss through the insulation is given by:

$$
\dot{Q}_{\text {loss }}=A_{\text {s,insul }}\left(T_{s}-T_{a m b}\right) / R
$$

Where:

$$
R=\frac{r_{2}}{k_{\text {insul }}} \ln \left(\frac{r_{2}}{r_{1}}\right)
$$


$r_{1}$ and $r_{2}$ are the inner and outer radii of the insulation respectively, with $r_{1}=0.0333 \mathrm{~m}$ and $r_{2}=0.1633 \mathrm{~m}$.

Because the amount of heat loss through the insulation is known, the above Equation 5.3 can be altered by subtracting the amount of heat lost through insulation $\left(\dot{Q}_{\text {loss }}\right)$ from the power input $\left(\dot{Q}_{\text {in }}\right)$ to give an improved energy balance estimation.

$$
E B_{\text {Improved }}=100 \frac{\left|\dot{Q}_{\text {in }}-\dot{Q}_{\text {loss }}-\dot{Q}_{\text {out }}\right|}{\frac{1}{2}\left(\left|\dot{Q}_{\text {in }}-\dot{Q}_{\text {loss }}\right|+\left|\dot{Q}_{\text {out }}\right|\right)}
$$

This improved energy balance estimation gives a maximum energy balance error of $2.6 \%$ for all cases.

\subsection{Thermocouple Wall Position Determination}

Because stainless steel has a relatively low thermal conductivity, there was a significant radial temperature gradient inside the wall during diabatic conditions. Therefore, because the wall thermocouples at $m=2$ to $m=6$ were installed in the grooves of the stainless steel tube and not on the wetted surface, the measured thermocouple values could not be used directly to determine the wetted wall temperatures.

In order to account for the installed position of the measured thermocouples, a semianalytical/empirical/experimental technique was used to determine the temperature difference between each thermocouple tip and its associated wetted wall position. As is shown in Figure 5-1 for a particular measuring station $m$, the thermocouples were not necessarily positioned at the idealized radial position at the bottom of the groove (Fig 5-1a) as was initially intended, but in reality (Fig 5-1b) each thermocouple tip probably had a different radial position (depending on the exact groove depth, its exact installed position and its exact size and shape). Such variations would lead to the different thermocouples reading different temperatures even if the wetted wall temperature was uniform. Therefore, the impact of possible radial variations had to be calibrated separately for each thermocouple at each measuring station.

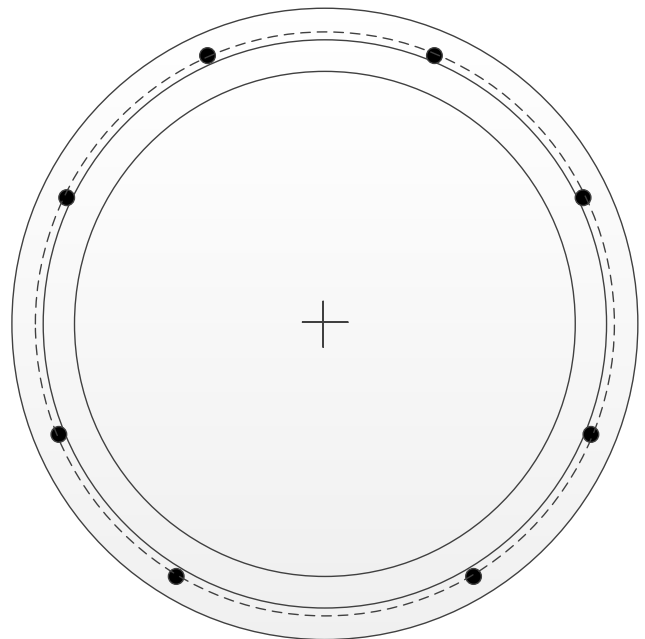

a)

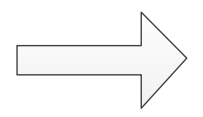

Figure 5-1: Exaggerated actual thermocouple positions and the virtual thermocouple positions due to the correction technique

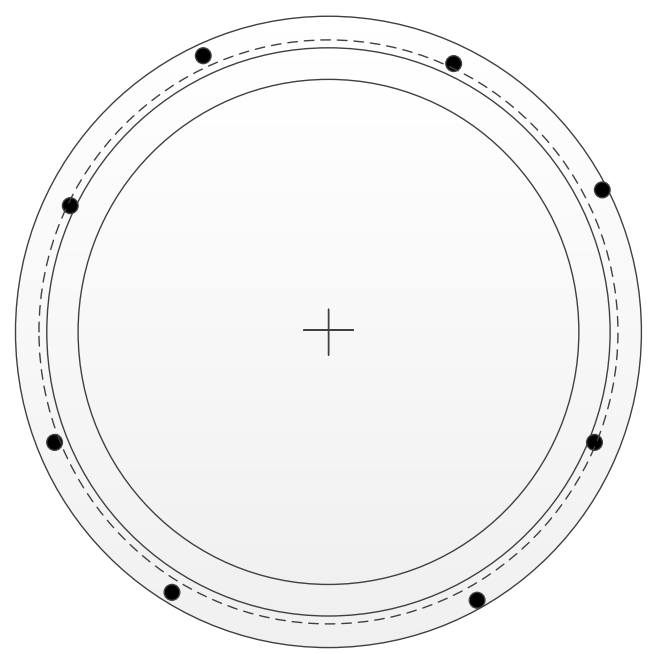

b) 
Firstly, the installed radial positions of each thermocouple needed to be determined. This was necessary since even though the groove depth within the tube wall was relatively small, a significant temperature difference existed across it in the radial direction. A control volume was drawn around each of the measuring stations for the purpose of the calculations to follow (as seen in Figure 5-2).

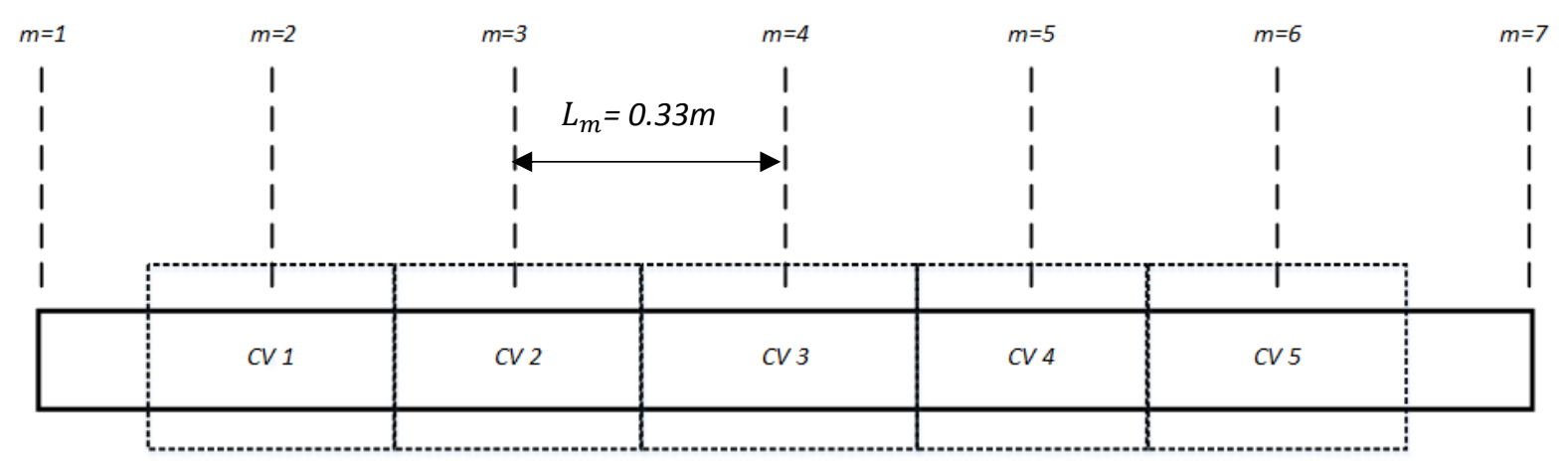

Figure 5-2: Control volumes for each of the measuring stations

Positional calibration data was collected experimentally for uniform heated conditions with all the heating elements being energized with a total heat input $(\dot{Q})$ of approximately $800 \mathrm{~W}$. Highly turbulent flow conditions ( $\mathrm{Re}=10396$ ) were used such that only forced convection conditions were present $\left(\mathrm{Ri}=2.8 \times 10^{-5}\right)$. The methods used for determining the heat transfer rate $(\dot{Q})$, Reynolds number (Re) and Richardson number (Ri) are described in more detail in the data reduction chapter later in the dissertation.

The turbulent nature of the flow meant that the wetted surface had a circumferentially uniform wall temperature at each axial location for any selected constant radial coordinate in the tube wall. Thus, the relative temperature differences between the thermocouples at $n=1$ to $n=8$ for a particular station $m$ could be used to determine the different radial thermal resistance variations associated with each thermocouple installation. This was done by making use of the steady state heat conduction equations for a radial system as is given by Equation 5.6.

$$
\dot{Q}_{m}=\frac{T_{T C, n}-T_{T C, a v g}}{R_{T C, n}}
$$

Here $T_{T C, n}$ is the corrected thermocouple measurements reading after the calibration correction was applied, $T_{T C, \text { avg }}$ is the arithmetic average of the wall thermocouples ( $n=1$ to 8 ) at that location (also after calibration correction), and $R_{T C, n}$ is the thermal resistance (difference) between the actual installed radial position of the thermocouple and the average installed radial position at that location $m$.

$R_{T C, n}$ can be expressed as follows:

$$
R_{T C, n}=\frac{\ln \left(\frac{r_{T C, n}}{r_{T C, a v g}}\right)}{2 \times \pi \times k \times L_{m}}
$$

Here $r_{T C, n}$ is the expected radial position of an individual thermocouple tip, $r_{T C, \text { avg }}$ is the expected arithmetic average of the radius of the thermocouple tips ( $n=1$ to 8 ). $L_{m}$ is the distance between two data measuring stations $(m)$. 
An average radial distance for all 8 thermocouples at each measuring station was assumed based on the photographs taken at every thermocouple installation. The assumed average radial distance was the radius of the grooves made for the installation of the thermocouples, plus half the diameter of the thermocouple tip, with half a millimeter added for any movement that may have occurred during installation.

The calibrated thermocouple temperatures, the average of the thermocouple temperatures at each measuring station, the assumed average radial distance for each thermocouple as well as the amount of energy supplied to each control volume was used to find the actual radial position of each measuring thermocouple by combining Equation 5.6 and Equation 5.7 and making $r_{T C, n}$ the subject:

$$
r_{T C, n}=r_{T C, a v g} \times e^{\left(2 \times \pi \times L_{m} \times k \times \frac{T_{T C, n}-T_{T C, a v g}}{\dot{Q}_{m}}\right)}
$$

Once the actual radial distance for each thermocouple was calculated the sensitivity of the assumed average radial distances was checked. There was a $1.6^{\circ} \mathrm{C}$ difference between the wetted wall temperatures for the worst and best case scenarios of the radial thermocouple placement. The worst case was when the thermocouple tip is flush with the outer tube diameter and the best case scenario was when the thermocouple tip is perfectly flush with the bottom of the groove.

Table 5-1 lists the calculated actual radial distances $\left(r_{T C, n}\right)$ for each of the thermocouples $(n)$ at each measuring station $(m)$. The calculated radial positions for the thermocouples needed to be larger than the radial distance of the groove $(0.0144 \mathrm{~m})$ and less than the outer radial distance of the tube $(0.01667 \mathrm{~m})$. The radial positions for the thermocouples are a lot closer to the groove radius than the outer radius. This is expected due to where the thermocouples were connected.

Table 5-1: $r_{T C, n}$ values for each measuring station $(m)$

\begin{tabular}{|c|c|c|c|c|c|}
\hline & $m=2$ & $m=3$ & $m=4$ & $m=5$ & $m=6$ \\
\hline$n=1$ & 0.01512 & 0.01505 & 0.01539 & 0.01525 & 0.01512 \\
\hline$n=2$ & 0.01518 & 0.01501 & 0.01504 & 0.01519 & 0.01518 \\
\hline$n=3$ & 0.01509 & 0.01502 & 0.01515 & 0.01506 & 0.01503 \\
\hline$n=4$ & 0.01501 & 0.01524 & 0.01521 & 0.01503 & 0.01512 \\
\hline$n=5$ & 0.01511 & 0.01514 & 0.01517 & 0.01521 & 0.01515 \\
\hline$n=6$ & 0.01512 & 0.01508 & 0.01512 & 0.01514 & 0.01509 \\
\hline$n=7$ & 0.01502 & 0.01503 & 0.01508 & 0.01512 & 0.01521 \\
\hline$n=8$ & 0.01521 & 0.01504 & 0.01515 & 0.01524 & 0.01525 \\
\hline
\end{tabular}

\subsection{Tangential Heat Conduction}

An analytical model was required to determine the relative tangential heat conduction rates when non-uniform heat flux cases were considered. Heat input from a particular heating element was not necessarily transferred in only the radial direction, but depending on whether the neighboring heating elements were powered or not, a significant proportion of the heat could have been transferred tangentially. This is despite the choice of stainless steel as tube material and its low thermal conductivity. The model was based on the assumption of one-dimensional heat transfer in the tangential direction inside the tube wall. For this purpose, the tube wall was divided into 8 different sectors to match the heating element strip positions. Figure 5-3 shows how the tube was divided into the different sectors $(n)$ to allow for one-dimensional heat transfer to be considered as well as an assumed uniform hypothetical heat transfer coefficient on the inner surface $\left(h^{*}\right)$. 


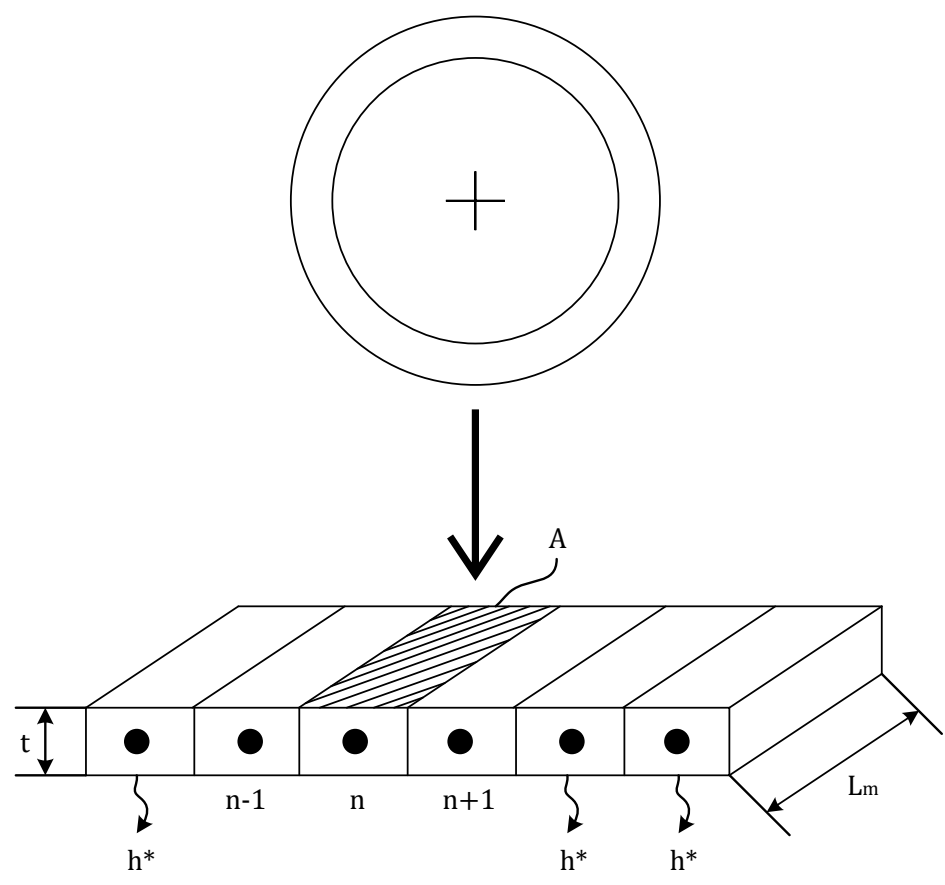

Figure 5-3: Converting the tube wall to a one-dimensional strip and discretising it into different sections

The tube wall was divided into 8 circumferential sectors to match the heating element strip positions. For wall sector $n$, the energy balance principle can be used to relate the incoming heat transfer rate expressed in terms of the local heating element heat flux $\left(\dot{q}_{i n, n} A_{n}\right)$ with the heat transfer rate to the bulk fluid temperate, as well as the conductive heat transfer rates to the neighbouring wall sectors $n+1$ and $n-1$. By making use of the combined radial conduction and convection thermal resistance $\left(R_{\text {rad }}\right)$ and the tangential conduction thermal resistances $\left(R_{n-1}\right.$ and $\left.R_{n+1}\right)$, the following steady state equation can be employed to express the local wall temperature $\left(T_{n}\right)$ in terms of an adopted bulk fluid temperature $\left(T_{B}^{*}\right)$ and the adjacent wall temperatures $\left(T_{n+1}\right.$ and $\left.T_{n-1}\right)$ :

$$
\begin{gathered}
\frac{T_{n-1}-T_{n}}{R_{n-1}}+\frac{T_{n+1}-T_{n}}{R_{n+1}}+\dot{q}_{i n, n} A_{n}+\frac{T_{B}^{*}-T}{R_{\text {rad }}} \\
\text { Where: } \\
A_{n}=\frac{\pi D_{\text {mid }} L_{m}}{8} \\
R_{n-1}=R_{n+1}=\frac{\pi D_{\text {mid }}}{8 k t L_{m}} \\
R_{\text {rad }}=\frac{4 t}{k \pi D_{\text {mid }} L_{m}}+\frac{8}{h^{*} \pi D_{\text {mid }} L_{m}} \\
D_{\text {mid }}=D+\frac{t}{2}
\end{gathered}
$$

The same can be done for all the sectors resulting in a simple set of 8 linear equations that can be solved for any combination of energized heating elements for an assumed hypothetical internal heat transfer coefficient $\left(h^{*}\right)$ and an assumed bulk fluid temperature $\left(T_{B}^{*}\right)$. For each possible condition, the 
percentage of the heat transferred $(\dot{Q} \%)$ by each sector to the water in terms of the local applied incoming heat flux from the local energized heating element can be found as follows:

$$
\dot{Q} \%=100 \times\left(\frac{\dot{Q}_{n}}{\dot{Q}_{i n, n}}\right)
$$

Where:

$$
\begin{gathered}
\dot{Q}_{i n, n}=\dot{q}_{i n, n} A_{n} \\
\dot{Q}_{n}=\frac{\left(T_{B}^{*}-T_{n}\right)}{R_{\text {rad }}}
\end{gathered}
$$

Where $\dot{Q}_{i n, n}$ is the applied heat transfer rate from the external heating element and $\dot{Q}_{n}$ is the corrected heat transfer rate on the inner wall for each sector based on the solved set of equations.

This resulted in an analytically obtained database which could be consulted for any heating element "powered-on" combination to determine what the effective radial heat transfer rates would be in each of the sectors ( $n=1$ to $n=8$ ) around the tube. Combined with the calibrated thermocouple radial positions, this allowed for the local wetted wall temperatures to be determined from the measured thermocouple values.

$$
T_{w, n}=T_{\text {original }}-\dot{Q}_{n}\left(R_{T C, n}\right)
$$

Where $T_{\text {original }}$ is the thermocouple reading which has only had the thermal calibration applied and does not take the thermocouple position or tangential heat conduction into account.

Table 5-2 gives the radial heat flux correction percentages for each of the different cases.

Table 5-2: Radial heat flux correction values

\begin{tabular}{|c|c|c|c|c|c|c|c|c|}
\hline \multirow{2}{*}{ Sector } & \multicolumn{7}{|c|}{ Local internal radial heat flux as a percentage of the externally applied heat flux } \\
\cline { 2 - 9 } & \multicolumn{7}{|c|}{ Case numbers } \\
\cline { 2 - 9 } & $1,2,3,4$ & $5,6,7$ & $8,9,10$ & 11 & 12 & 13 & 14 & 15,16 \\
\hline$n=1$ & $100 \%$ & $12.3 \%$ & $87.7 \%$ & $3.9 \%$ & $64.7 \%$ & $73.1 \%$ & $26.9 \%$ & $26.9 \%$ \\
\hline$n=2$ & $100 \%$ & $26.9 \%$ & $73.1 \%$ & $8.5 \%$ & $23 \%$ & $87.7 \%$ & $12.3 \%$ & $73.1 \%$ \\
\hline$n=3$ & $100 \%$ & $73.1 \%$ & $26.9 \%$ & $23 \%$ & $8.5 \%$ & $87.7 \%$ & $12.3 \%$ & $87.7 \%$ \\
\hline$n=4$ & $100 \%$ & $87.7 \%$ & $12.3 \%$ & $64.7 \%$ & $3.9 \%$ & $73.1 \%$ & $26.9 \%$ & $87.7 \%$ \\
\hline$n=5$ & $100 \%$ & $87.7 \%$ & $12.3 \%$ & $64.7 \%$ & $3.9 \%$ & $26.9 \%$ & $73.1 \%$ & $73.1 \%$ \\
\hline$n=6$ & $100 \%$ & $73.1 \%$ & $26.9 \%$ & $23 \%$ & $8.5 \%$ & $12.3 \%$ & $87.7 \%$ & $26.9 \%$ \\
\hline$n=7$ & $100 \%$ & $26.9 \%$ & $73.1 \%$ & $8.5 \%$ & $23 \%$ & $12.3 \%$ & $87.7 \%$ & $12.3 \%$ \\
\hline$n=8$ & $100 \%$ & $12.3 \%$ & $87.7 \%$ & $3.9 \%$ & $64.7 \%$ & $26.9 \%$ & $73.1 \%$ & $12.3 \%$ \\
\hline
\end{tabular}

Figure 5-4 gives the graphical representation of the radial heat flux correction percentage values for cases 5,6 and 7 . 

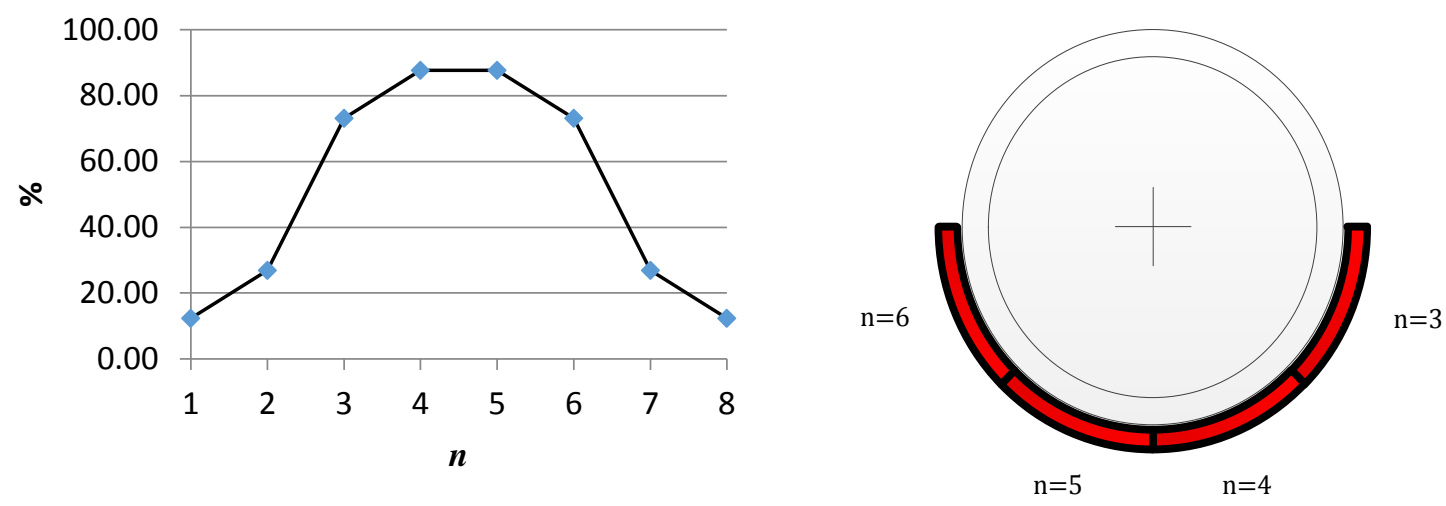

Figure 5-4: Radial heat flux correction percentage graphically represented for cases 5, 6 and 7

\subsection{Nusselt Numbers and Heat Transfer Coefficients}

The wetted wall temperature values were obtained at each measuring position in the pipe wall by first calculating the relative thermal resistance $\left(R_{T C, n}\right)$ for each thermocouple using Equation 5.7. Since the secondary flow was negligible, a circumferentially uniform wall temperature could be achieved at each axial position $(m)$. The actual wetted wall temperature at each measuring station is given by:

$$
T_{T C, m}=T_{T C, n}-\left(\dot{Q}_{m} \times R_{T C, n}\right)
$$

The average log mean temperature difference between the wall and fluid was calculated using:

$$
\Delta \bar{T}_{L M T D}=\frac{\left(T_{w, 7}-T_{B, 7}\right)-\left(T_{w, 1}-T_{B, 1}\right)}{\ln \left(\frac{T_{w, 7}-T_{B, 7}}{T_{w, 1}-T_{B, 1}}\right)}
$$

The applicable average wall temperatures (local or overall) were calculated as the arithmetic average for stations $m=2$ to $m=6$ and sectors $n=1$ to $n=8$. This was done due to there being no measuring stations at $m=1$ to $m=7$ (for reasons stated earlier). $T_{w, 1}$ and $T_{w, 7}$ were found by using a polynomial line equation (order 2 ) fitted to the known wetted wall temperatures and substituting their relative axial positions $\left(T_{w, 1}=0 \mathrm{~m}\right.$ and $\left.T_{w, 7}=2 \mathrm{~m}\right)$ to find their associated wetted wall temperatures. The axial relationship of the wetted wall temperatures and their axial positions is further explained in Figure 6-1, Figure 6-2 and Figure 6-3. With near linear relationships seen for these wetted wall temperature profiles a polynomial equation of order 2 was deemed sufficient to accurately predict the outlying wetted wall temperatures.

The local bulk fluid temperatures, $T_{B, 1}$ and $T_{B, 7}$ were assumed to be equal to $T_{B, 0}$ and $T_{B, 8}$ respectively. The bulk fluid temperatures were measured at $m=0$ to $m=8$ (at the mixing stations). The heat transferred between $m=0$ and $m=1$, and $m=7$ and $m=8$ was calculated to be sufficiently low (due to the fitted insulation) to make the above assumption.

The effective average heat transfer coefficient was found by using the calculated heat transfer rate, the full internal tube surface area and the temperature difference.

$$
\bar{h}=\frac{\dot{Q}_{\text {out }}}{A_{s} \Delta \bar{T}_{L M T D}}
$$

The effective Nusselt number was based on the calculated heat transfer coefficient, the inner diameter of the tube and the thermal conductivity of the water, $k$ (based on the equations of Popiel and Wojtkowiak (1998)), evaluated at the average bulk temperature. 


$$
\overline{\mathrm{Nu}}=\frac{\bar{h} D}{k}
$$

Since the heat flux being added to the system is axially uniform the local Nusselt numbers were found for a number of cases. The local Nusselt numbers were found using the log mean temperature difference.

$$
\Delta T_{L M T D}=\frac{\left(T_{w, m+1}-T_{B, m+1}\right)-\left(T_{w, m}-T_{B, m}\right)}{\ln \left(\frac{T_{w, m+1}-T_{B, m+1}}{T_{w, m}-T_{B, m}}\right)}
$$

The Nusselt numbers were found for each control volume mentioned earlier. This means the local heat transfer coefficient was found using the area for the control volume, $A_{m}$, the heat flux for the control volume, $Q_{m}$, and the log mean temperature difference above.

$$
h_{m}=\frac{\dot{Q}_{m}}{A_{m} \Delta T_{L M T D}}
$$

Where:

$$
A_{m}=\pi D_{i} L_{m}
$$

The local Nusselt number was then calculated using:

$$
\mathrm{Nu}_{m}=\frac{\mathrm{h}_{m} D_{i}}{k}
$$

\subsection{Colburn j-Factor}

The Colburn j-factor is an analogy between momentum, mass and heat transfer. It is also used as a tool that is used to show the transition of fluid flow from the laminar to the turbulent regime.

$$
j=\frac{\mathrm{Nu}}{\operatorname{Re} \times \operatorname{Pr}^{\frac{1}{3}}}
$$

The Prandtl number was calculated using the equations given by Popiel and Wojtkowiak (1998) at the bulk fluid temperature.

\subsection{Reynolds Number}

The Reynolds number is defined as the ratio of inertial forces to viscous forces for a given flow, given by:

$$
\operatorname{Re}=\frac{\rho V D_{i}}{\mu}
$$

The density $(\rho)$ and dynamic viscosity $(\mu)$ were found by evaluating the equations of Popiel and Wojtkowiak (1998) at the bulk fluid temperature. The velocity $(V)$ of the fluid was found by the equation:

$$
V=\frac{\dot{m}}{\rho A_{\text {cross }}}
$$


Where the mass flow rate $(\dot{m})$ was logged from the mass flow meters themselves and $A_{\text {cross }}$ was the cross sectional area of the inside of the tube.

\subsection{Grashof Number}

The Grashof number was found using $9.81 \mathrm{~m} / \mathrm{s}^{2}$ as the gravitational constant $(g)$, the coefficient for thermal expansion ( $\beta$ ) was found using the equations of Popiel and Wojtkowiak (1998) at the average bulk fluid temperature. The kinematic viscosity $(v)$ was found by finding the dynamic viscosity $(\mu)$ and density $(\rho)$ using the equations of Popiel and Wojtkowiak (1998) at the bulk fluid temperature and then dividing the dynamic viscosity by the density. The arithmetic averages of the wetted wall temperatures (explanation found above) and bulk temperatures were found, and with the measured diameter $\left(D_{i}\right)$, all the variables were known and the Grashof number could be calculated using:

$$
\mathrm{Gr}=\frac{g \beta\left(\overline{T_{w}}-\overline{T_{B}}\right) D_{i}^{3}}{v^{2}}
$$

\subsection{Richardson Numbers}

The Richardson number was found by dividing the calculated Grashof number by the Reynolds number squared:

$$
\mathrm{Ri}=\frac{\mathrm{Gr}}{\mathrm{Re}^{2}}
$$

\subsection{Uncertainty and Sensitivity Analysis Overview}

When taking readings and calculating different parameters there are certain inherent uncertainties which are caused by inaccuracies in the measuring equipment. The measurement uncertainties and propagated uncertainties are below.

\subsubsection{Instruments}

\subsubsection{Thermocouples}

As mentioned, PT-100 temperature probes were used in order to calibrate the thermocouples using the in situ method of calibration. The PT-100 probes had an accuracy of $0.1^{\circ} \mathrm{C}$ based on the manufacturers specifications. Data points were recorded for each thermocouple and plotted against the readings from the PT-100 probes for different temperatures, and from this calibration curves were found.

The accuracy of the Pt-100 probes was used as the bias, and the precision of the thermocouples was found using the equations found in Appendix A. The uncertainties of the thermocouples was then found using Equation 5.24.

$$
\delta x=\left(b_{i}^{2}+p_{p_{i}}^{2}\right)^{\frac{1}{2}}
$$

The uncertainties for the thermocouples was found to be between $0.1^{\circ} \mathrm{C}$ and $0.15^{\circ} \mathrm{C}$.

\subsubsection{Coriolis Flow Meter}

The Coriolis flow meter had a measuring range of 0 to $0.604 \mathrm{~kg} / \mathrm{s}$ and an accuracy of $0.05 \%$. Therefore, the CFM025 had a capacity of $2174 \mathrm{l} / \mathrm{h}$ and accuracy of 1.087 . 


\subsubsection{Power Supply}

The accuracy of the $3 \mathrm{~kW}$ DC power supply used was given in the technical data sheet supplied by the company. The power supply had an accuracy of $0.15 \%$ of the nominal value for both the voltage and current.

\subsubsection{Fluid Properties}

The uncertainty of the fluid properties can be calculated using the equations put forward by Popiel and Wojtkowiak (1998).

Table 5-3: Uncertainties of the fluid properties (Popiel and Wojtkowiak, 1998)

\begin{tabular}{|l|c|}
\hline Property & Uncertainty \\
\hline $\boldsymbol{C}_{p}[\mathrm{~J} / \mathrm{kgK}]$ & $0.04 \%$ \\
\hline $\boldsymbol{\mu}[\mathrm{kg} / \mathrm{ms}]$ & $1 \%$ \\
\hline $\boldsymbol{k}[\mathrm{W} / \mathrm{mK}]$ & $2 \%$ \\
\hline $\boldsymbol{\rho}\left[\mathrm{kg} / \mathrm{m}^{3}\right]$ & $0.004 \%$ \\
\hline $\operatorname{Pr}[-]$ & $2.3 \%$ \\
\hline $\boldsymbol{\beta}[1 / \mathrm{K}]$ & $0.073 \%$ \\
\hline
\end{tabular}

\subsubsection{Dimensions}

The accuracy of the dimensions was determined by the instruments used to measure the respective dimensions. The length and the diameter were measured using a vernier calliper. The length was measured using measuring tape.

Table 5-4: Dimension accuracies

\begin{tabular}{|l|c|}
\hline Dimension & Accuracies \\
\hline $\boldsymbol{t}[\mathrm{m}]$ & $20[\mu \mathrm{m}]$ \\
\hline $\boldsymbol{D}[\mathrm{m}]$ & $20[\mu \mathrm{m}]$ \\
\hline $\boldsymbol{L}[\mathrm{m}]$ & $1[\mathrm{~mm}]$ \\
\hline
\end{tabular}

\subsubsection{Calculated Parameters}

The method used for finding the measurement uncertainties in the calculated parameters was that of Dunn (Dunn, 2010). The equations used to find the uncertainties can be found in Appendix A. A summary of the uncertainties of the instruments, fluid properties and dimensions can be found above. These were used to find the uncertainties of the calculated properties (Reynolds numbers, heat transfer coefficients and Nusselt numbers).

The Reynolds number range was between $650-2600$. The uncertainty was found to be virtually constant throughout the range, and was just above $1 \%$. There is little difference between the fluid properties throughout the Reynolds number as well as heat input ranges. There is also very little difference in uncertainty found in the flow meter for the specified Reynolds number range. And the accuracy of the diameter is constant. With the Reynolds number being a function of pressure, dynamic viscosity, velocity and diameter, it makes sense then that there is little change in the difference of uncertainty throughout the Reynolds range.

The heat transfer coefficient is a function of the heat flux and the temperature and was seen to increase across the range of Reynolds number and heat flux from $2.4 \%$ to $2.65 \%$. An estimation for the uncertainty associated with the position of the thermocouple tip is around $3 \%$. This estimation is 
based on the uncertainties of the fluid and material properties, measured dimensions and the equations used to find the radial positioning for each thermocouple tip.

The Nusselt number is a function of the heat transfer coefficient, diameter and thermal conductivity of the fluid and therefore an increase in uncertainty was also seen throughout the Reynolds number and heat flux ranges. The same is evident for the local heat transfer coefficient.

Table 5-5: Uncertainties of calculated parameters

\begin{tabular}{|l|c|c|}
\hline Parameter & $\mathbf{R e}=\mathbf{6 5 0}, \mathbf{Q}=\mathbf{3 0 0} \mathbf{W}$ & $\mathbf{R e}=\mathbf{2 6 0 0}, \mathbf{Q}=\mathbf{8 0 0 W}$ \\
\hline $\mathbf{R e}$ & $1.02 \%$ & $1.03 \%$ \\
\hline $\boldsymbol{h}$ & $2.4 \%$ & $2.65 \%$ \\
\hline$\overline{\mathbf{N u}}$ & $3.36 \%$ & $3.6 \%$ \\
\hline $\mathbf{N u}_{\mathbf{m}}$ & $3.36 \%$ & $3.6 \%$ \\
\hline
\end{tabular}

\subsection{Summary of Chapter}

This chapter contains the data reduction methods used to find the energy balances, Nusselt numbers and heat transfer coefficients. The method to account for tangential heat transfer in the pipe is also included. The overview for the uncertainty and sensitivity analyses is found in this chapter. In the next chapter the experimental results are given. 


\section{Experimental Results}

\subsection{Introduction}

The experimental results gathered in this section are precluded by a few necessary verifications and validations. The verification of the heating elements and the determination of the critical Reynolds number, where the onset of transition occurs, were first investigated. The laminar, turbulent and local heat transfer coefficients were then validated. The boundary case results are then presented. These include the axial wall temperature profiles, cases with the same heat flux and cases with the same in going heat transfer rates. The effect of the Richardson number on the Nusselt number was then investigated. Followed by what effect the position of the incident incoming heat flux had on the Richardson number and Nusselt number. Finally, Nusselt number correlations for $360^{\circ}$, lower $180^{\circ}$ and upper $180^{\circ}$ heating cases are presented.

\subsection{Verifications}

A number of verifications were performed to determine whether the set-up and the test procedure produced meaningful result. Firstly, heating element validation was done, then the range of Reynolds numbers constituting laminar flow was identified, and lastly it was checked whether the obtained Nusselt numbers were comparable with the results from prior investigations from literature.

\subsubsection{Heating Element Verification}

To ensure that the heating elements were all supplying the system with the same amount of heat, a few tests needed to be performed. Eight different test cases were examined, one for each heating element. Each test case had only one of the heating elements switched on at a time, had a Reynolds number of 4700 , and had a power input of $260 \mathrm{~W}$. The relatively high Reynolds number (when compared to the rest of the cases found in this dissertation) was chosen to ensure that the system was not in the laminar flow regime. A higher Reynolds number and higher power input would have been ideal, as the higher Reynolds number would ensure turbulent flow and good mixing of the flow, and a higher power input would give a larger change in temperature between the inlet and outlet temperatures. However, the power input needed to be limited to ensure that the heating element did not burn out. Therefore, the Reynolds number was lowered to still have a meaningful difference between the inlet and outlet temperature. Table 6-1 contains the difference between the inlet and outlet temperatures for each of the 8 heating element tests.

Table 6-1: Inlet and outlet temperature differences for different heating elements

\begin{tabular}{|c|c|}
\hline Heating Element & Temperature Difference $(\Delta T)$ \\
\hline 1 & $0.917^{\circ} \mathrm{C}$ \\
\hline 2 & $0.902^{\circ} \mathrm{C}$ \\
\hline 3 & $0.896^{\circ} \mathrm{C}$ \\
\hline 4 & $0.918^{\circ} \mathrm{C}$ \\
\hline 5 & $0.897^{\circ} \mathrm{C}$ \\
\hline 6 & $0.902^{\circ} \mathrm{C}$ \\
\hline 7 & $0.899^{\circ} \mathrm{C}$ \\
\hline 8 & $0.895^{\circ} \mathrm{C}$ \\
\hline
\end{tabular}

There is a maximum temperature difference of $0.023^{\circ} \mathrm{C}$ between the highest and the lowest temperature differences (resulting in a maximum difference of $2.5 \%$ ). Therefore, the heating elements were said to all be performing the same and no further calibration was needed. 
To ensure the walls of the tube were heated uniformly (for uniform heating cases) and that the actual wetted wall temperature was being measured a few axial wetted wall temperature profiles will be presented. These profiles are for cases 1 (uniform heating with a total heat input of $800 \mathrm{~W}$ ), 2 (uniform heating with a total heat input of $600 \mathrm{~W}$ ) and 3 (uniform heating with a total heat input of $400 \mathrm{~W}$, see Table 4-1). Three different Reynolds numbers are chosen for each case to show the wetted wall temperature profiles at different mass flow rates.

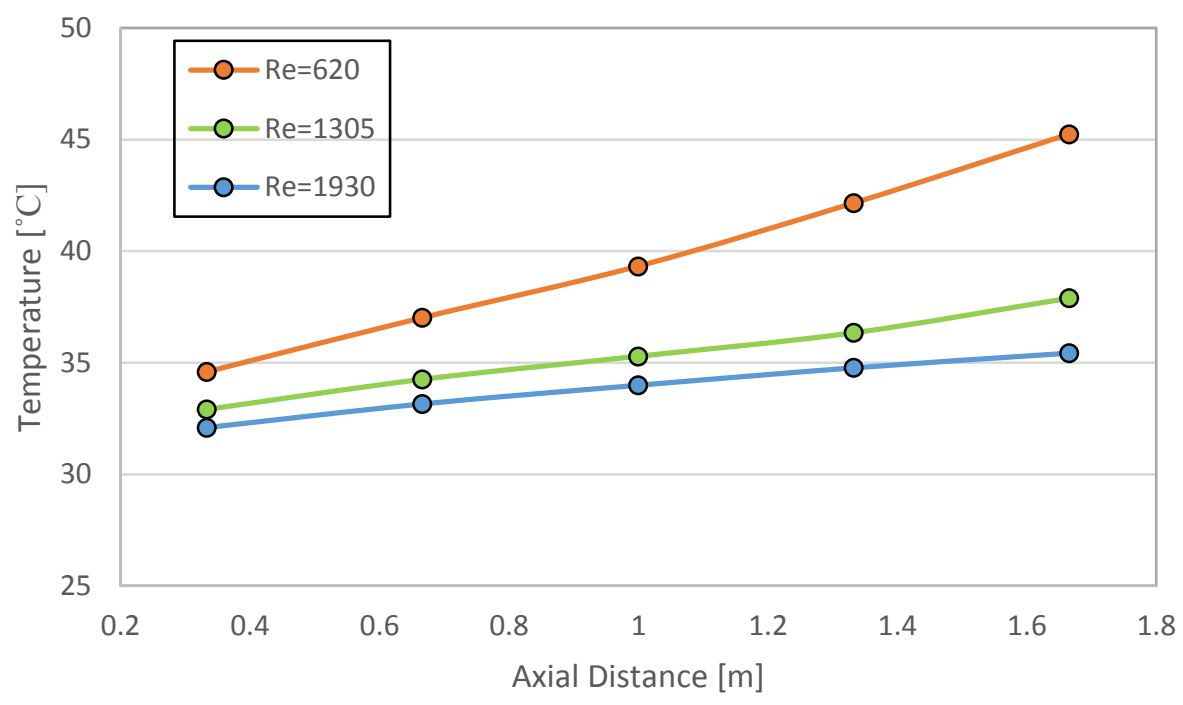

Figure 6-1: Axial wetted wall temperature profiles for case 1

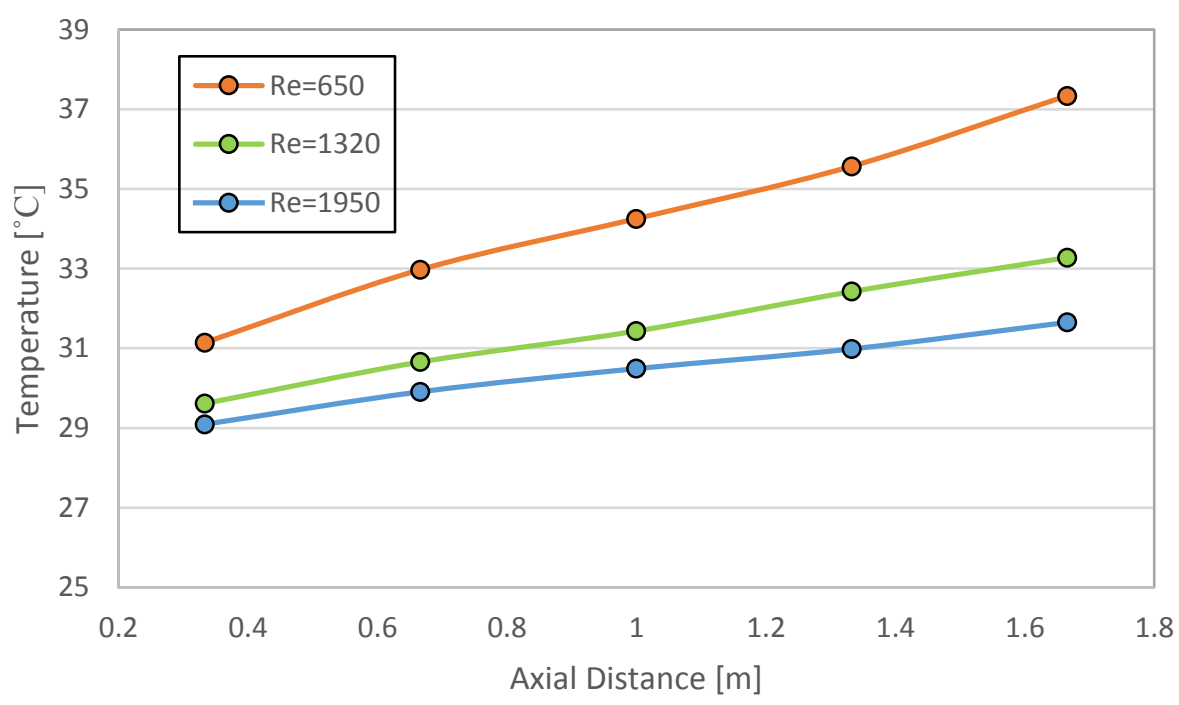

Figure 6-2: Axial wetted wall temperature profiles for case 2 


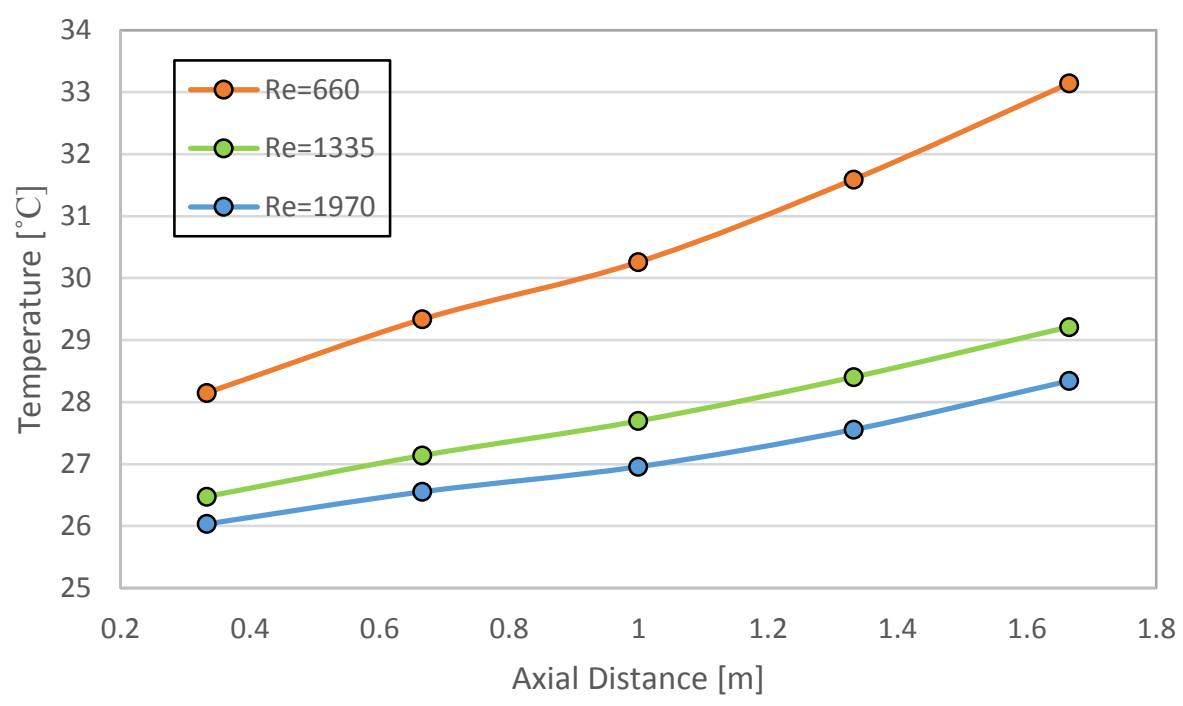

Figure 6-3: Axial wetted wall temperature profiles for case 3

Figure 6-1, Figure 6-2 and Figure 6-3 all show smooth approximately linear increments for the wetted wall temperatures along the length of the tube. These cases were chosen to show that the linear relationship is not significantly affected by the change in Reynolds number and the amount of heat added to the system.

\subsubsection{Onset of Transition}

The onset of the transitional flow regime was determined by conducting experiments over a Reynolds number range from approximately 600 to 4500 at uniform heat flux conditions. For this purpose, case 2 (uniform heat flux with a total heat transfer rate of $600 \mathrm{~W}$ ) as defined in Table 4-1 was used.

Figure 6-4 shows the average Nusselt number against the Reynolds number. It can be seen that for a Reynolds number range between 600 and approximately 3000, that the Nusselt number is relatively constant, and that for Reynolds numbers above 3000 a significant increase in the Nusselt number is observed. Based on this, it was found that the onset of the transitional flow regime occurred at Reynolds numbers of approximately $3000-3500$. Similarly, Figure 6-5 shows the Colburn j-factor versus the Reynolds numbers. A significant change in the gradient of the $\mathrm{j}$-factors is evident at a Reynolds number of approximately $3000-3500$. Based on this, the upper bound of the Reynolds number in the test matrix was selected to be 2600 (as mentioned earlier) because it sufficiently lower than the expected critical Reynolds number. Thus, all subsequent data presented in this dissertation falls within the laminar flow regime. 


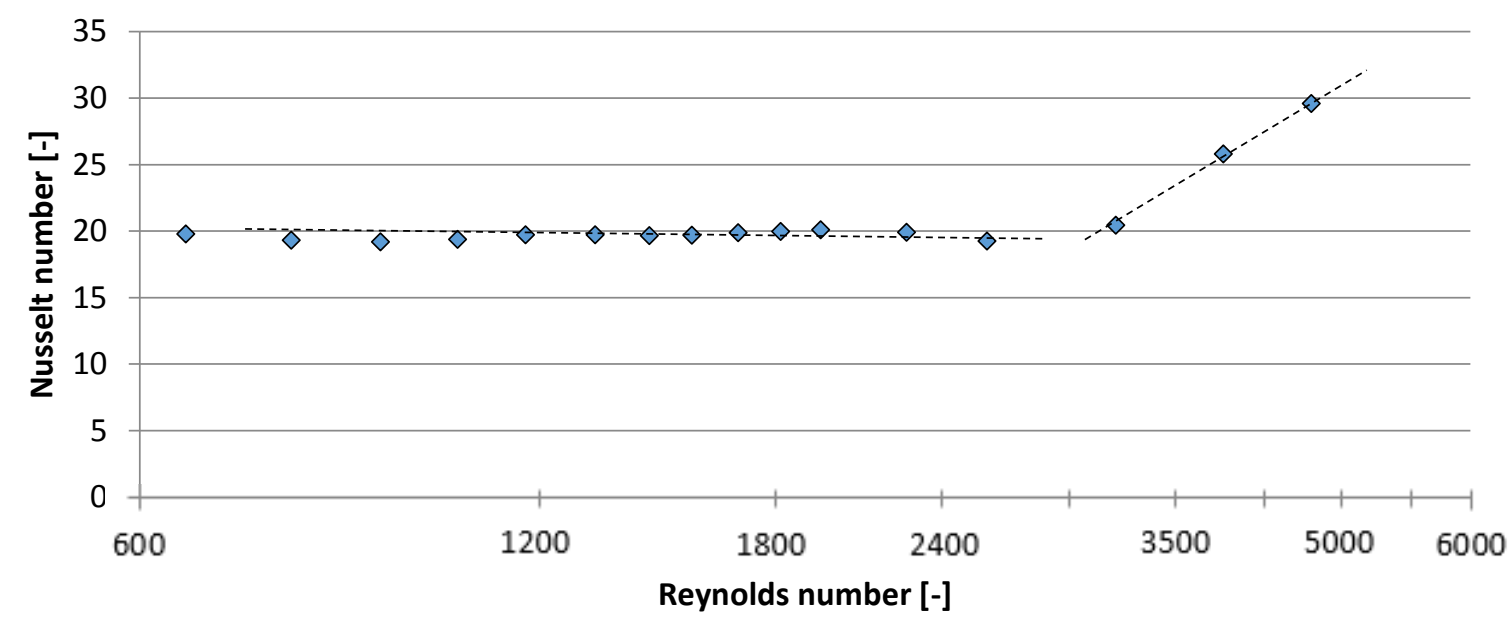

Figure 6-4: Nusselt number vs. Reynolds number showing the change from laminar to the transitional flow regime

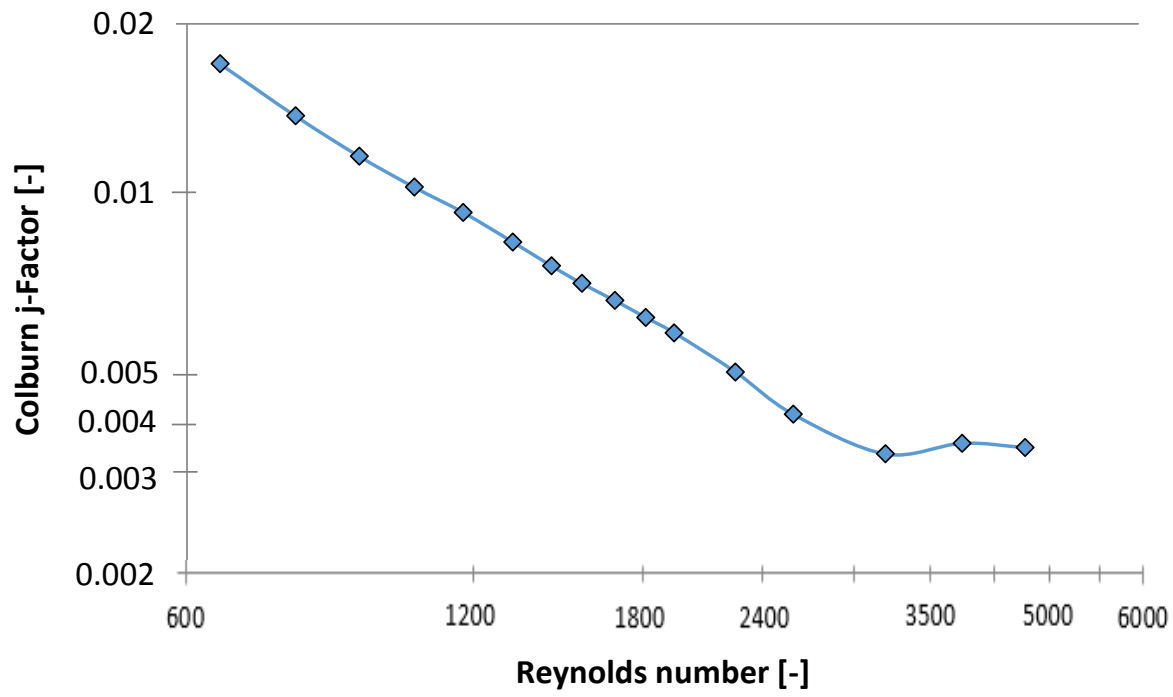

Figure 6-5: Colburn j-Factor vs. Reynolds number showing the change from laminar to the transitional flow regime

\subsubsection{Laminar Comparisons with Correlations from Literature}

To verify whether the test setup produced meaningful results, experimental data for case 1 (uniform heating with a total heat input of $800 \mathrm{~W}$ ) was compared against correlations from literature that were specifically developed for uniform heat flux. This was done for Reynolds numbers ranging from 650 to 1950.

Once the laminar regime Reynolds number test range was confirmed, experimentally obtained Nusselt numbers were compared with relevant correlations from literature (Figure 6-6). For this purpose the correlations by Ghajar and Tam (Ghajar and Tam, 1994) and Morcos and Bergles (Morcos and Bergles, 1975) were selected because the conditions for these cases were similar to those found in this study. A comparison with the fully developed forced convection Nusselt number of 4.36 was also included to show the effect that mixed convection can have on the heat transfer and how one cannot simply assume fully developed forced convection. In order to do these comparisons, data that was obtained from case 1 is presented in Figure 6-6. The experimental results also include error bars of around 3.4\% 
to account for the uncertainty in the data capturing process and the associated uncertainty propagation. It can be seen that the experimental Nusselt numbers are approximately $9.6 \%$ higher than the Ghajar and Tam predictions, $41.4 \%$ higher that the Marcos and Bergles predictions and $128 \%$ higher than the fully developed forced convection Nusselt number of 4.36 . It is clear that the differences are significantly greater than the measurement uncertainty.

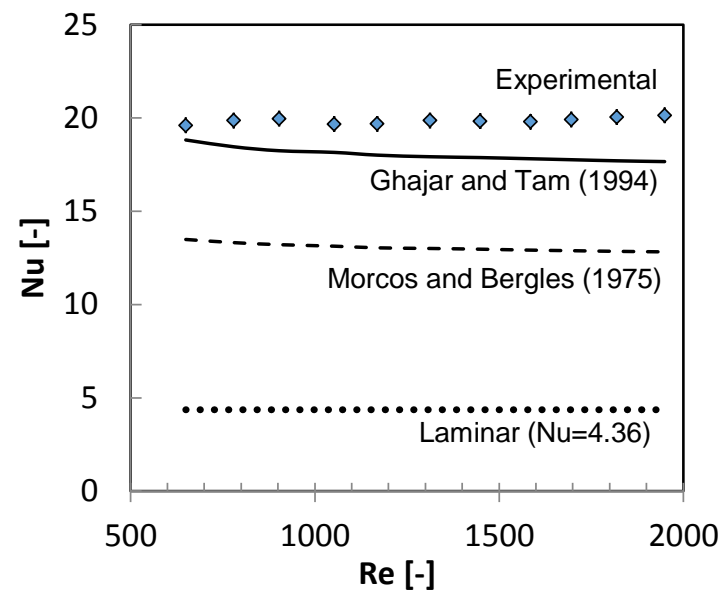

Figure 6-6: Comparison of laminar data from case 1 to previous works

Although the Nusselt numbers obtained in this investigation were higher than the Nusselt numbers predicted by the correlations, some justification for this can be given.

In the case of Ghajar and Tam (Ghajar and Tam, 1994) equation 2.4 was used. The difference between the experimental data and the predictions of this correlation can be attributed to the mismatch between the Grashof and Prandtl number ranges. The correlation was developed from data with a Grashof range of 1000 to $2.5 \times 10^{5}$ and for a Prandtl number range from 40 to 160 while for the data shown in Figure 6-6, the Grashof and Prandtl number ranges were $9.95 \times 10^{7}$ to $1.29 \times 10^{8}$ and 5.48 to 6.46 respectively. The reason for the difference in the predicted Nusselt numbers could be due to the fact that the Ghajar and Tam correlations were not developed with water as the working fluid (Prandtl number range). Thus, allowing for a certain discrepancy between the results.

When considering the Morcos and Bergles correlation (Equation 2.3), a plausible difference is that the correlation is for fully developed flow (both hydrodynamically and thermally), while in this investigation the flow is still thermally developing, which is normally associated with higher heat transfer coefficients. Therefore, the development of the thermal profile could be the reason why the Nusselt number in this paper is larger than that of Morcos and Bergles.

\subsubsection{Turbulent Comparisons with Correlations from Literature}

Figure 6-4 and Figure 6-5 show that there is a transition to turbulence around a Reynolds number of 3000 . These tests used the data from case 2, where there was a uniform heat input of $600 \mathrm{~W}$ (see Table 4-1) and a Reynolds number range of 2600 to 4 555. The experimental data was compared to the Dittus-Boelter (Dittus and Boelter, 1985), the Sieder-Tate (Sieder and Tate, 1936) and Gnielinski (Gnielinski, 2010) correlations. Error bars of 3.8\% were added to the experimental data.

The Dittus-Boelter correlation is given as follows: 


$$
\mathrm{Nu}=0.023 \operatorname{Re}^{\frac{4}{5}} \operatorname{Pr}^{0.4}
$$

When the experimental data was compared to that of the Dittus-Boelter correlation an average deviation of $19.13 \%$ was observed. The difference could be due to the simplistic nature of this equation and the fact that the Dittus-Boelter correlation is designed for a Reynolds number range above 10000 .

The Gnielinski correlation is given as follows:

$$
\mathrm{Nu}=\frac{\frac{\mathrm{f}}{8}(\operatorname{Re}-1000) \operatorname{Pr}}{1+12.7\left(\frac{\mathrm{f}}{8}\right)^{\frac{1}{2}}\left(\operatorname{Pr}^{\frac{2}{3}}-1\right)}
$$

Where f:

$$
\mathrm{f}=(0.79 \ln (\mathrm{Re})-1.64)^{-2}
$$

When the experimental data was compared to that of the Gnielinski correlation an average deviation of $11.8 \%$ was observed. The Gnielinski correlation was expected to under predict the Nusselt number as this correlation is for fully developed flow (which yields lower Nusselt numbers).

The Sieder-Tate correlation is given as follows:

$$
\mathrm{Nu}=0.027 \operatorname{Re}^{\frac{4}{5}} \operatorname{Pr}^{\frac{1}{3}}\left(\frac{\mu}{\mu_{w}}\right)^{0.14}
$$

When the experimental data was compared to that of the Gnielinski correlation an average deviation of $2.53 \%$ was observed. This is within the experimental error bars added to the figure.

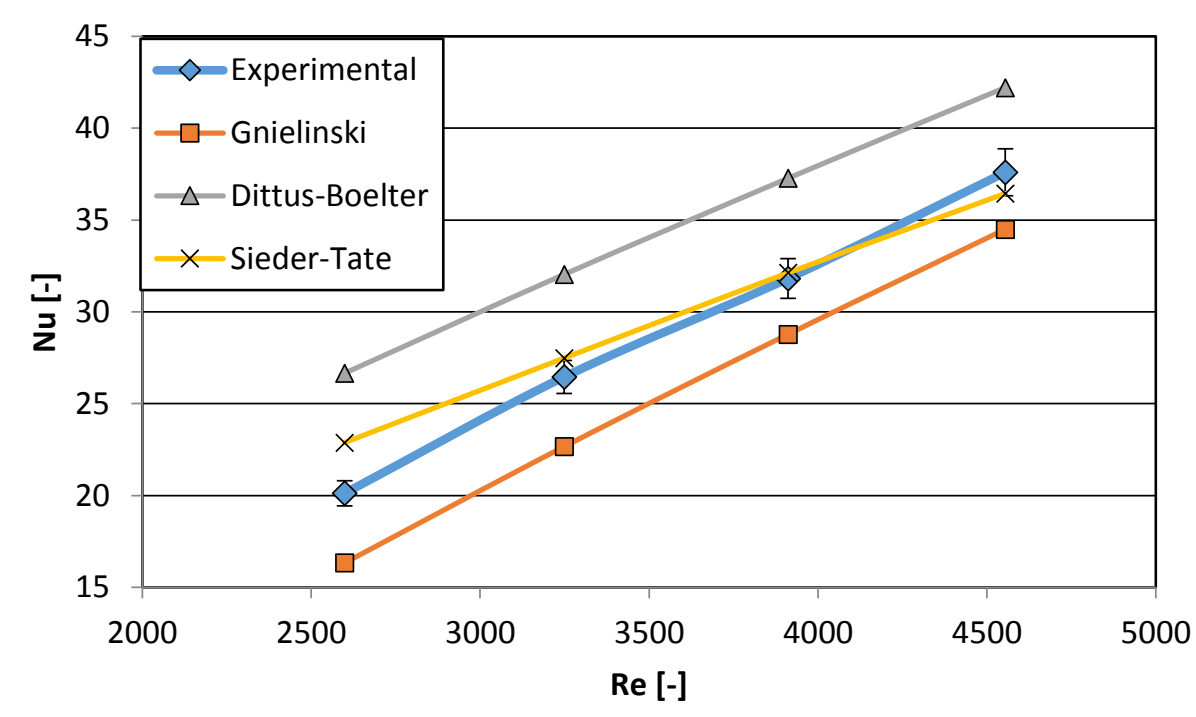

Figure 6-7: Comparison of turbulent results in case 2 to previous work 


\subsubsection{Local Nusselt Numbers}

The local Nusselt numbers found in this study were validated using the case of a Reynolds number of roughly 900 . The heat flux added was $4421 \mathrm{~W} / \mathrm{m}^{2}$ and the heating was circumferentially uniform. As seen in the previous sections this means that the flow is not in the purely forced convection region and mixed convection is present.

Figure 6-8 shows the validation of the data found in the present study with a number of different correlations found in literature. The general trend of all the data series is that the Nusselt numbers decrease along the length of the tube. This is seen to be true of the data in the present study. Although the literature shows predicted Nusselt numbers to be lower than the present study, the general region of Nusselt numbers is correct and the differences seen can, for the most part, be explained for each case.

The work from Ghajar and Tam (Ghajar and Tam, 1994) under predicts the present experimental data by a fair amount, as was seen in the Nusselt number calibration earlier. This could be due to the fact that the literature (Ghajar and Tam, 1994) used much higher Prandtl numbers than the Prandtl numbers used in this experiment. The over prediction for the Gauler (Gauler, 1972) data can be attributed to the fact that the correlation is for flow in the hydrodynamically developing region, which is not the case for the present study.

The correlation of Gnielinski (Gnielinski, 2010) is seen to produce much lower Nusselt numbers, this could be due to the fact that the Gnielinski correlation is for thermally fully developed flow. The present study has thermally developing flow and therefor the experimental results are expected to be higher. The experimental results are again higher than the 4.36 expected for fully developed forced convection laminar flow. This was expected due to the mixed convection present.

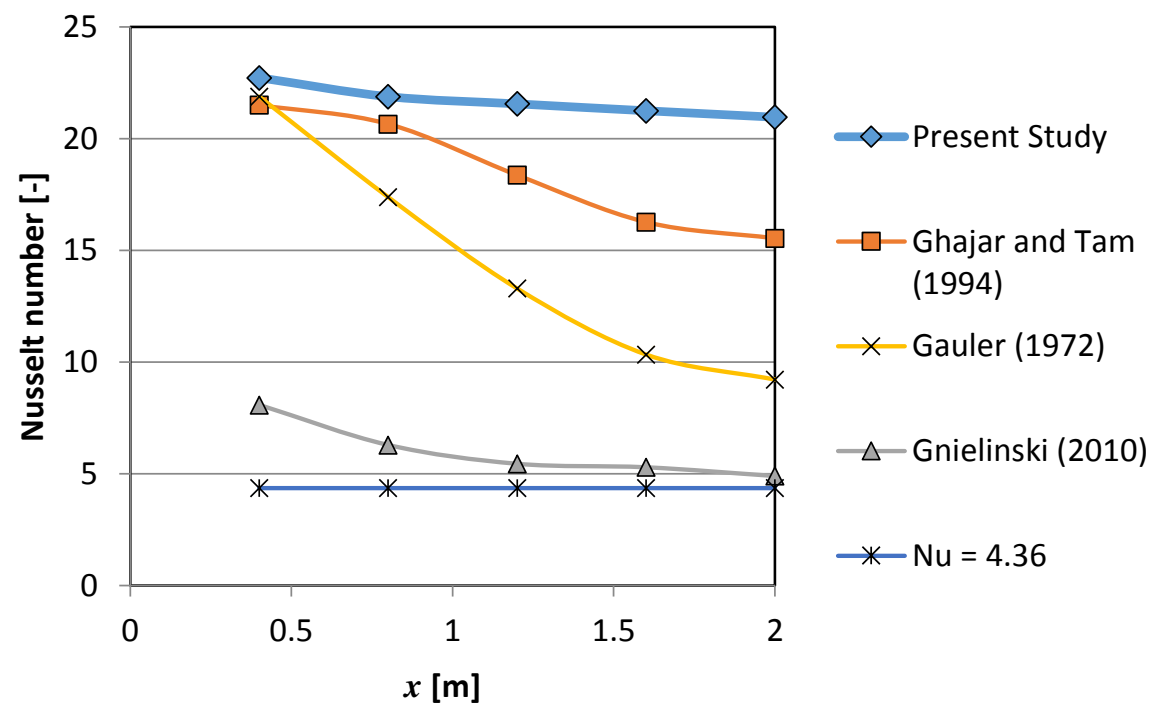

Figure 6-8: Local Nusselt number validation for $\operatorname{Re} \approx 900$ and a heat flux of $4421 \mathrm{~W} / \mathrm{m}^{2}$

\subsection{Boundary Cases Results}

\subsubsection{Axial Wall Temperatures}

This section gives the relative axial average wall temperature differences to the fluid temperature for all the cases in this study. The temperatures at each circumferential position $(n)$ are the average of their corresponding temperatures $\left(T_{n}\right)$ at $m=2$ to $m=6$. The temperature profiles are presented at two different Reynolds numbers, namely $\operatorname{Re}=650$ and $R e=1950$. It is seen that the difference between 
the relative axial average wall temperature and the fluid temperature is lower at higher Reynolds numbers. The reader is reminded that $n=1$ and $n=8$ are at the top of the tube while $n=4$ and $n=5$ are at the bottom of the tube. The bulk fluid temperatures at $m=2-5$ are calculated on an assumed linear relationship between the inlet and outlet temperatures, with their relative axial positions being substituted in order to find their bulk fluid temperature.

Figure 6-9, Figure 6-10, Figure 6-11 and Figure 6-12 are all for uniform heating cases. All the temperature profiles are symmetrical; however, it is observed that there is a decrease in the temperature difference between the wetted wall temperatures and the bulk fluid temperatures as the heat rate is decreased. This trait is observed through all the cases (where a heat rate comparison is possible).

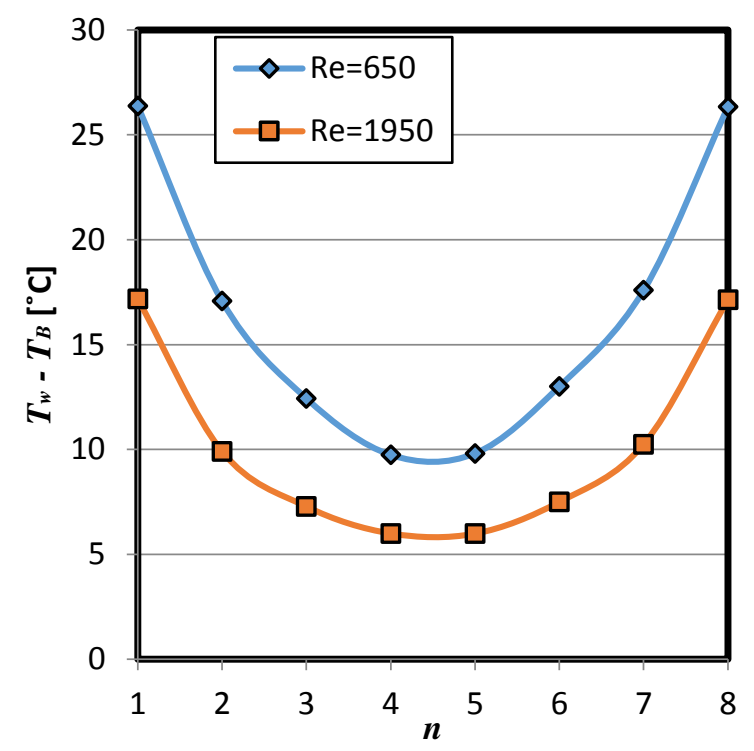

Figure 6-9: Temperature differences between the wetted wall temperatures and bulk fluid temperatures for case 1 $\left(360^{\circ}\right.$ heating at $\left.800 \mathrm{~W}\right)$

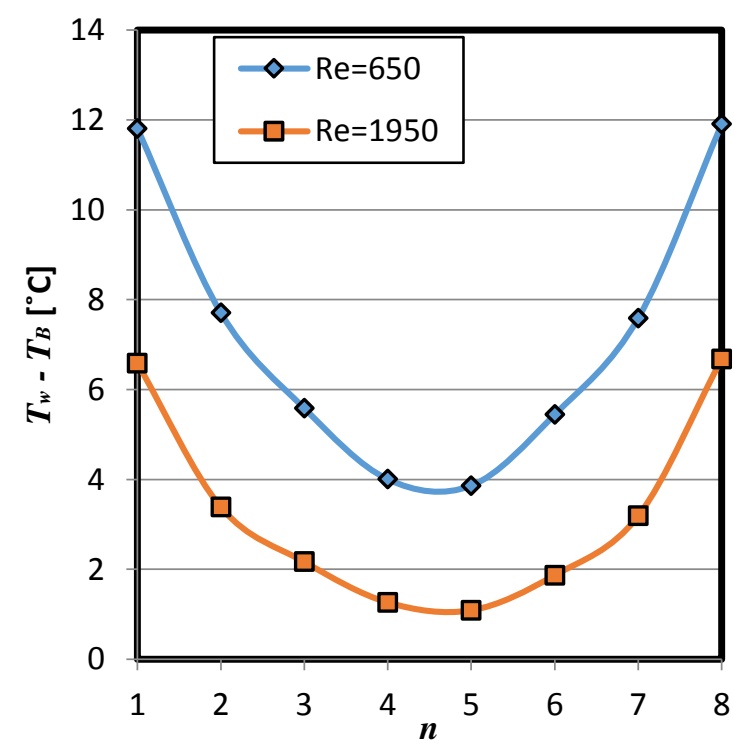

Figure 6-11: Temperature differences between the wetted wall temperatures and bulk fluid temperatures for case 3 $\left(360^{\circ}\right.$ heating at $\left.400 \mathrm{~W}\right)$

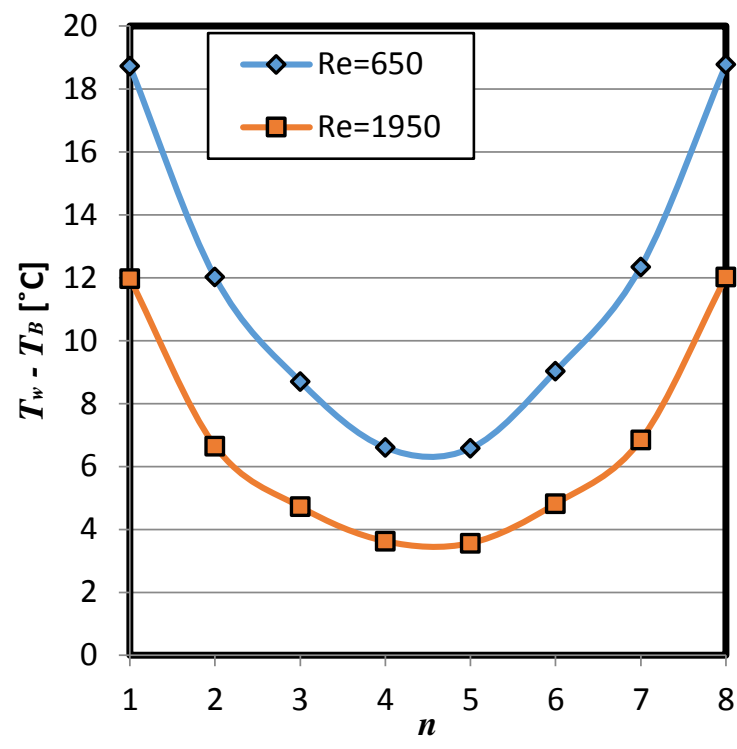

Figure 6-10: Temperature differences between the wetted wall temperatures and bulk fluid temperatures for case 2

$\left(360^{\circ}\right.$ heating at $\left.600 \mathrm{~W}\right)$

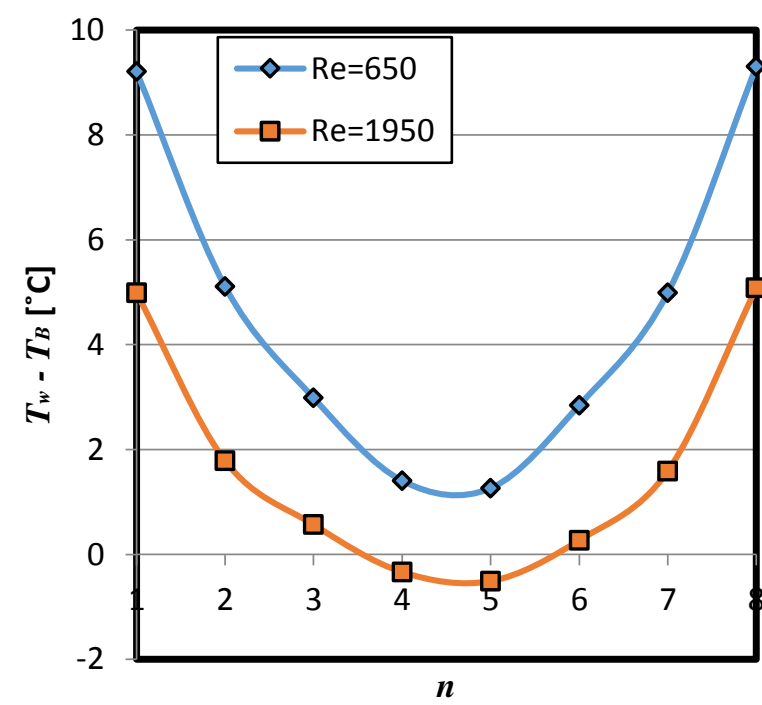

Figure 6-12: Temperature differences between the wetted wall temperatures and bulk fluid temperatures for case 4 $\left(360^{\circ}\right.$ heating at $\left.300 \mathrm{~W}\right)$ 
Figure 6-13,Figure 6-14 and Figure 6-15 show the temperature profiles for the lower $180^{\circ}$ heating cases. There is a much smaller difference between the maximum and minimum temperature differences when compared to the uniform heating cases. This is due to the secondary flow transferring the heat effectively between the lower and upper halves of the tube.

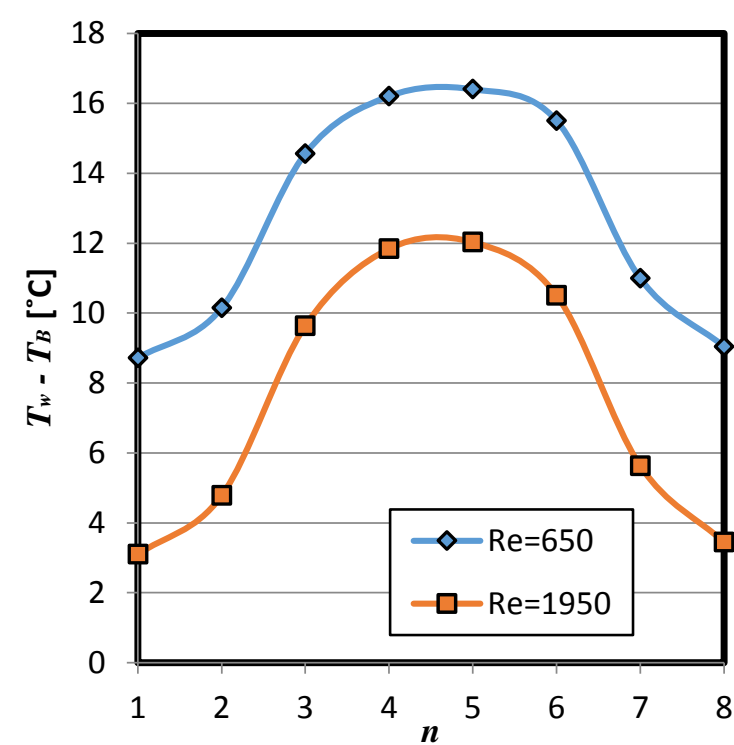

Figure 6-13: Temperature differences between the wetted wall temperatures and bulk fluid temperatures for case 5 (lower $180^{\circ}$ heating at $600 \mathrm{~W}$ )

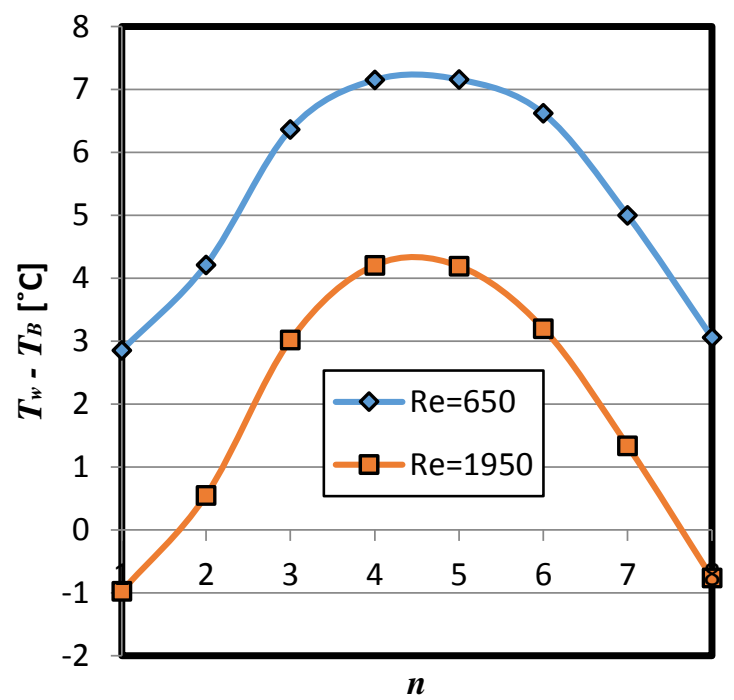

Figure 6-15: Temperature differences between the wetted wall temperatures and bulk fluid temperatures for case 7 (lower $180^{\circ}$ heating at $300 \mathrm{~W}$ )

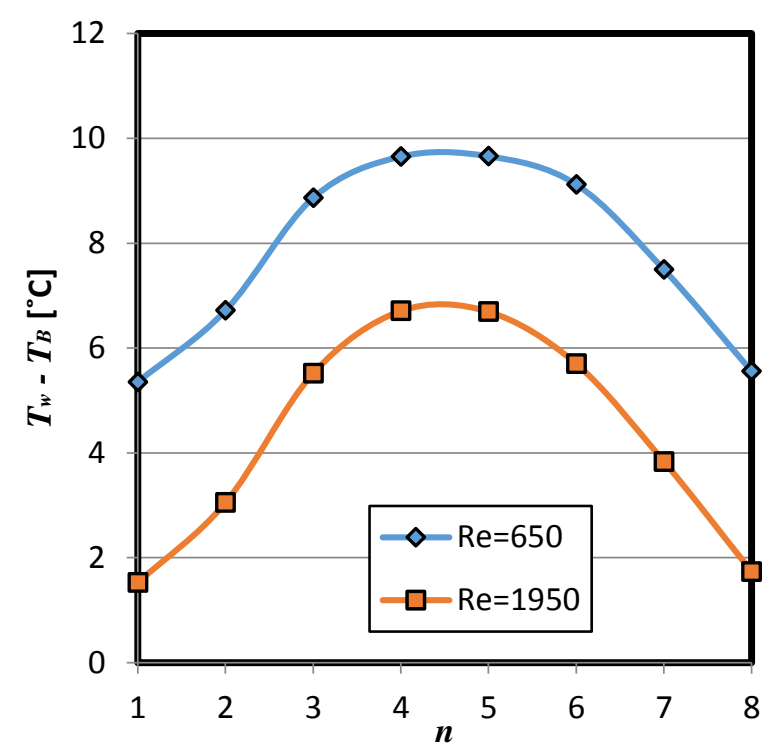

Figure 6-14: Temperature differences between the wetted wall temperatures and bulk fluid temperatures for case 6 (lower $180^{\circ}$ heating at $400 \mathrm{~W}$ ) 
Figure 6-16, Figure 6-17 and Figure 6-18 show the temperature profiles for the upper $180^{\circ}$ heating cases. These are similar to the uniform heating cases. The slopes on the profiles are more pronounced due to the buoyancy forces sustaining more of the heat transfer in the upper half of the tube.

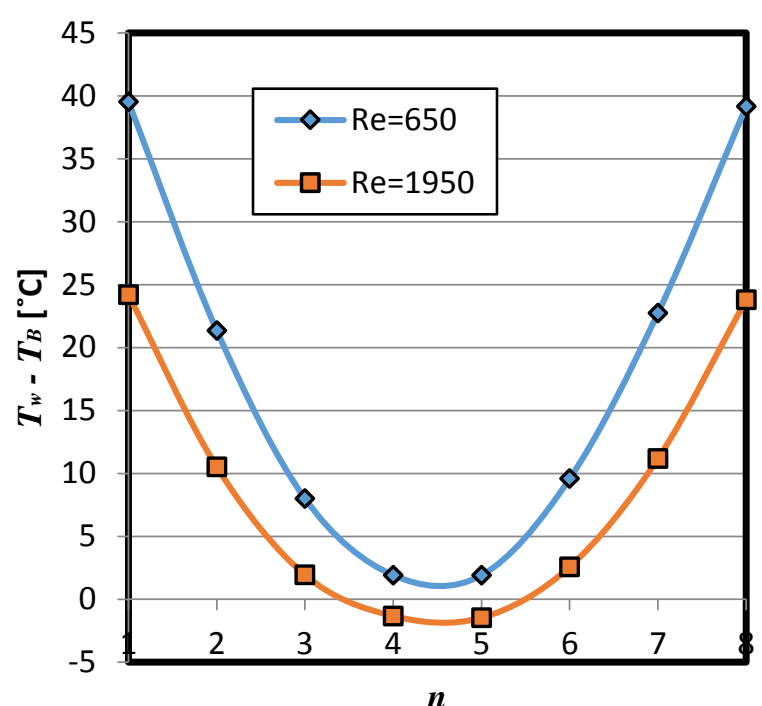

Figure 6-16: Temperature differences between the wetted wall temperatures and bulk fluid temperatures for case 8 (upper $180^{\circ}$ heating at $600 \mathrm{~W}$ )

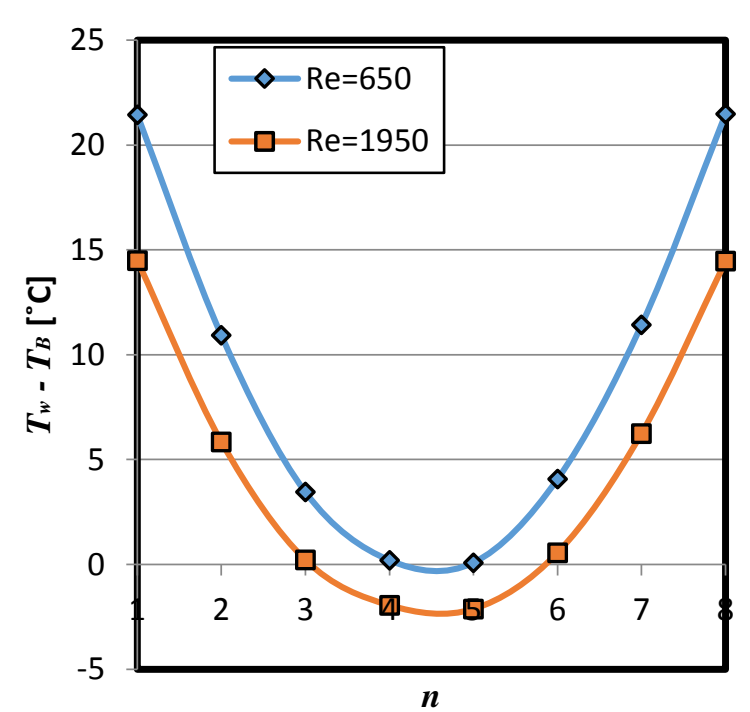

Figure 6-18: Temperature differences between the wetted
wall temperatures and bulk fluid temperatures for case 9

Figure 6-18: Temperature differences between the wetted
wall temperatures and bulk fluid temperatures for case 9 (upper $180^{\circ}$ heating at $400 \mathrm{~W}$ )

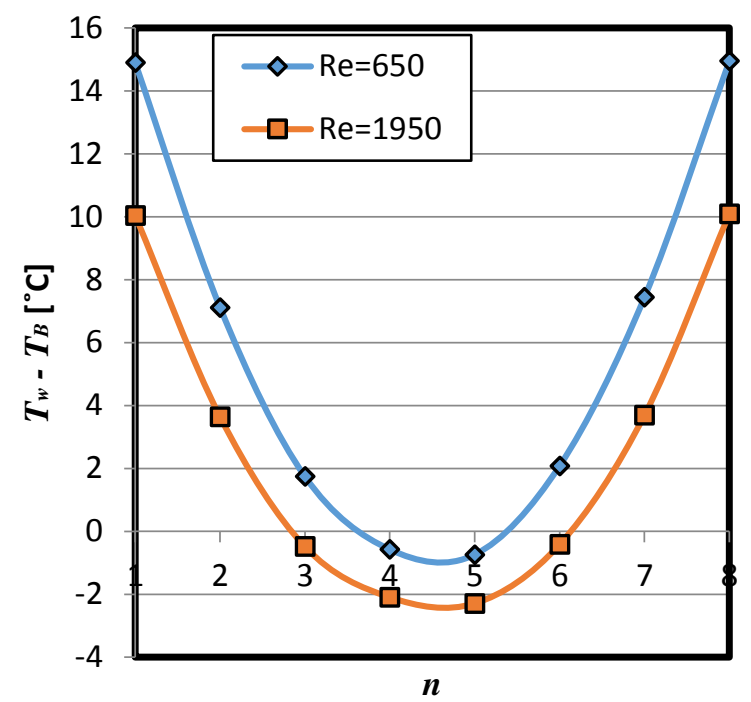

Figure 6-17: Temperature differences between the wetted wall temperatures and bulk fluid temperatures for case 10 (upper $180^{\circ}$ heating at $300 \mathrm{~W}$ )

Figure 6-19 and Figure 6-20 are for the lower $90^{\circ}$ and upper $90^{\circ}$ heating cases respectively. These cases are similar to their $180^{\circ}$ heating counterpart cases. However, it is seen that the slopes on the graphs are much more severe in the $90^{\circ}$ heating cases. This is to be expected due to the smaller angle span of heating. 


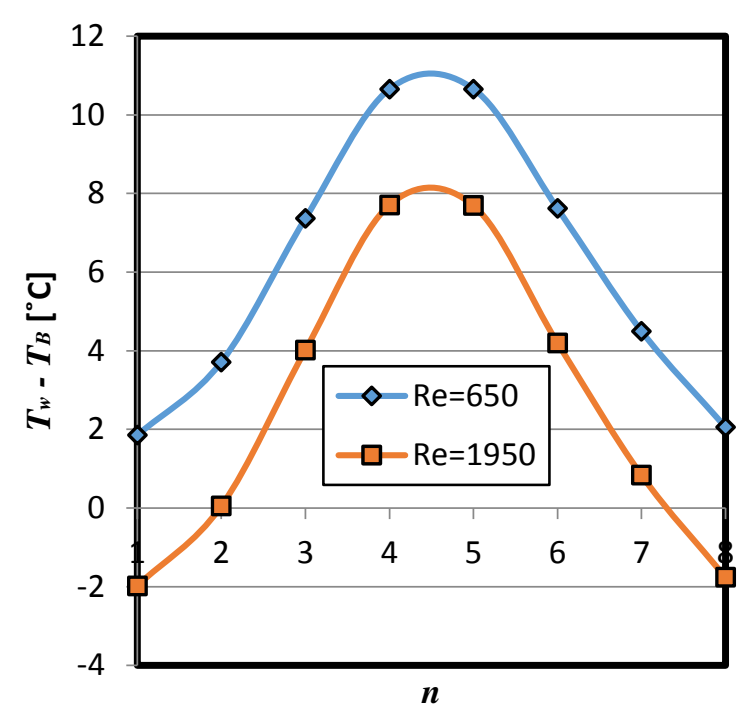

Figure 6-19: Temperature differences between the wetted wall temperatures and bulk fluid temperatures for case 11 (lower $90^{\circ}$ heating at $300 \mathrm{~W}$ )

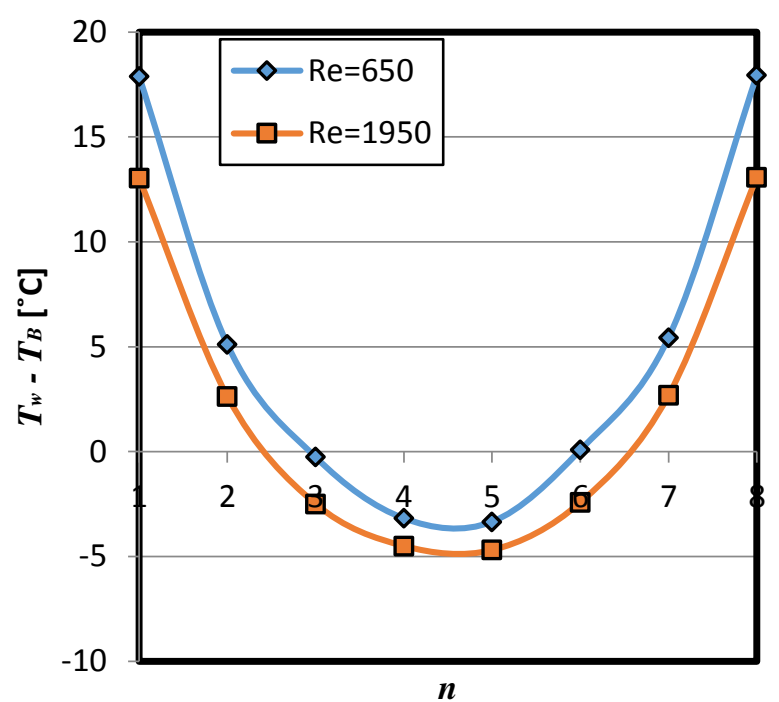

Figure 6-20: Temperature differences between the wetted wall temperatures and bulk fluid temperatures for case 12 (upper $90^{\circ}$ heating at $300 \mathrm{~W}$ )

Figure 6-21 and Figure 6-22 are the temperature profiles for the $180^{\circ}$ right heating and $180^{\circ}$ left heating respectively. It is seen that these profiles mirror each other almost exactly as expected.

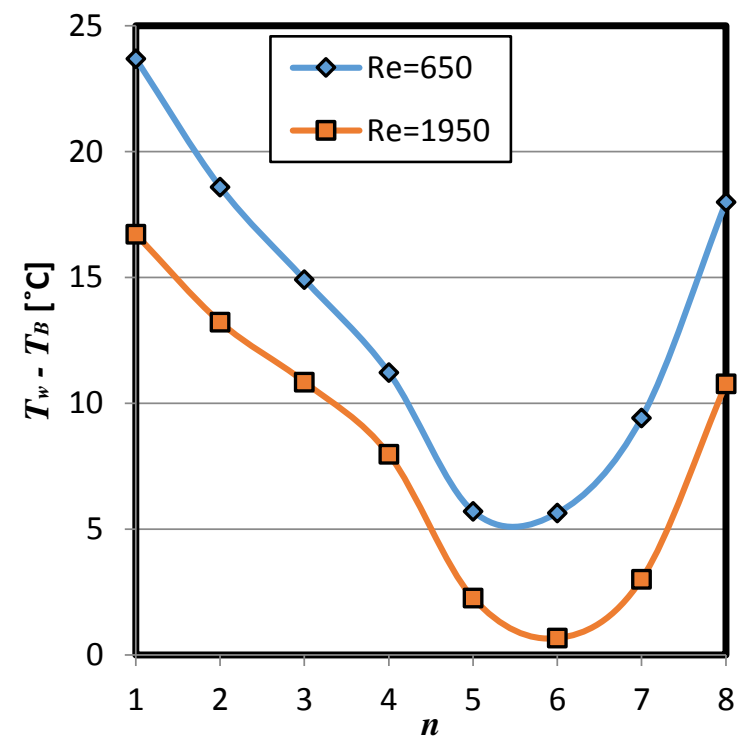

Figure 6-21: Temperature differences between the wetted wall temperatures and bulk fluid temperatures for case 13 (right $180^{\circ}$ heating at $600 \mathrm{~W}$ )

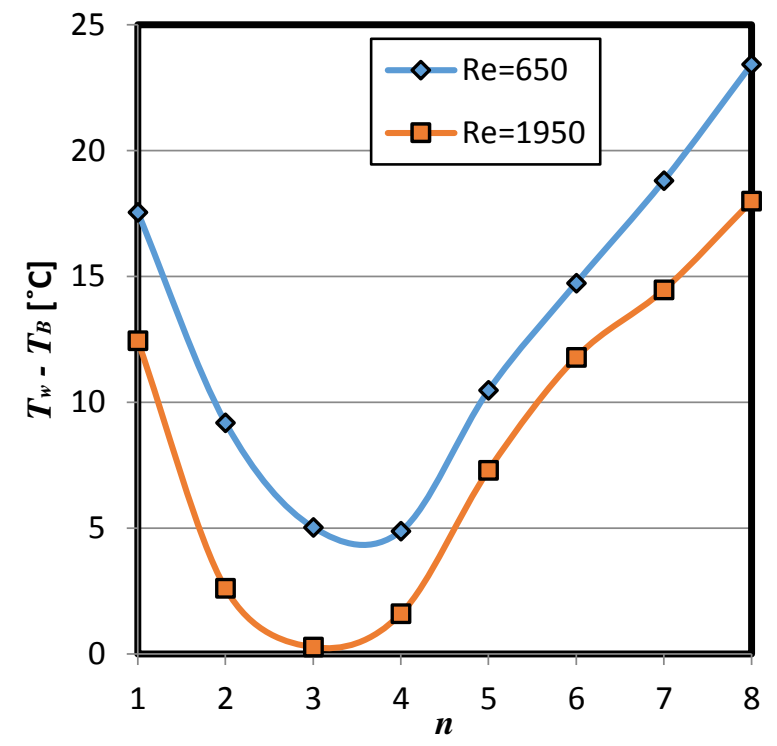

Figure 6-22: Temperature differences between the wetted wall temperatures and bulk fluid temperatures for case 14 (left $180^{\circ}$ heating at $600 \mathrm{~W}$ )

Figure 6-23 and Figure 6-24 show the temperature profiles for the slanted $180^{\circ}$ heating cases. The non-symmetrical nature of the profiles give a very clear indication of the buoyancy forces and secondary flow present in the flow. 


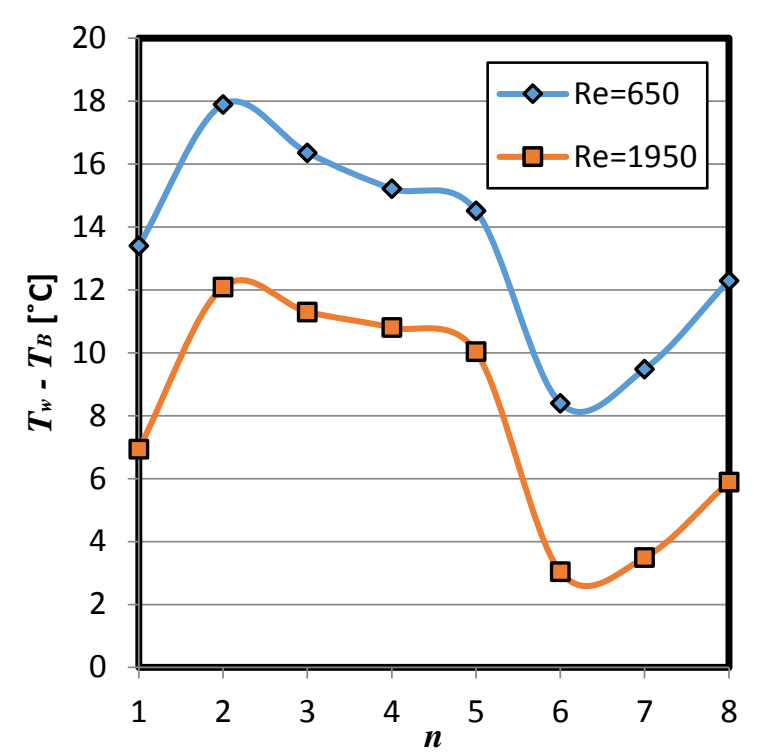

Figure 6-23: Temperature differences between the wetted wall temperatures and bulk fluid temperatures for case 15 (slanted $180^{\circ}$ heating at $600 \mathrm{~W}$ )

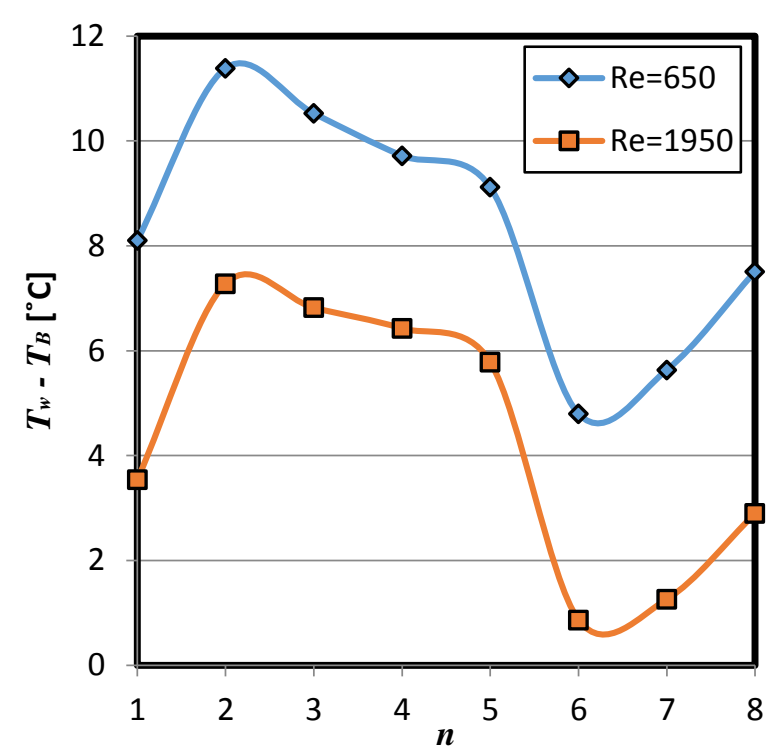

Figure 6-24: Temperature differences between the wetted wall temperatures and bulk fluid temperatures for case 16 (slanted $180^{\circ}$ heating at $400 \mathrm{~W}$ )

\subsubsection{Symmetry}

Figure 6-25 shows the symmetry in the results between the left and right heating cases at a heat flux of $6631 \mathrm{~W} / \mathrm{m}^{2}$. There is a maximum error of $3.7 \%$ between the cases. This is in-line with the expected experimental uncertainty. From this it can be deduced that only one of the symmetry conditions (left or right) need to be reported in full.

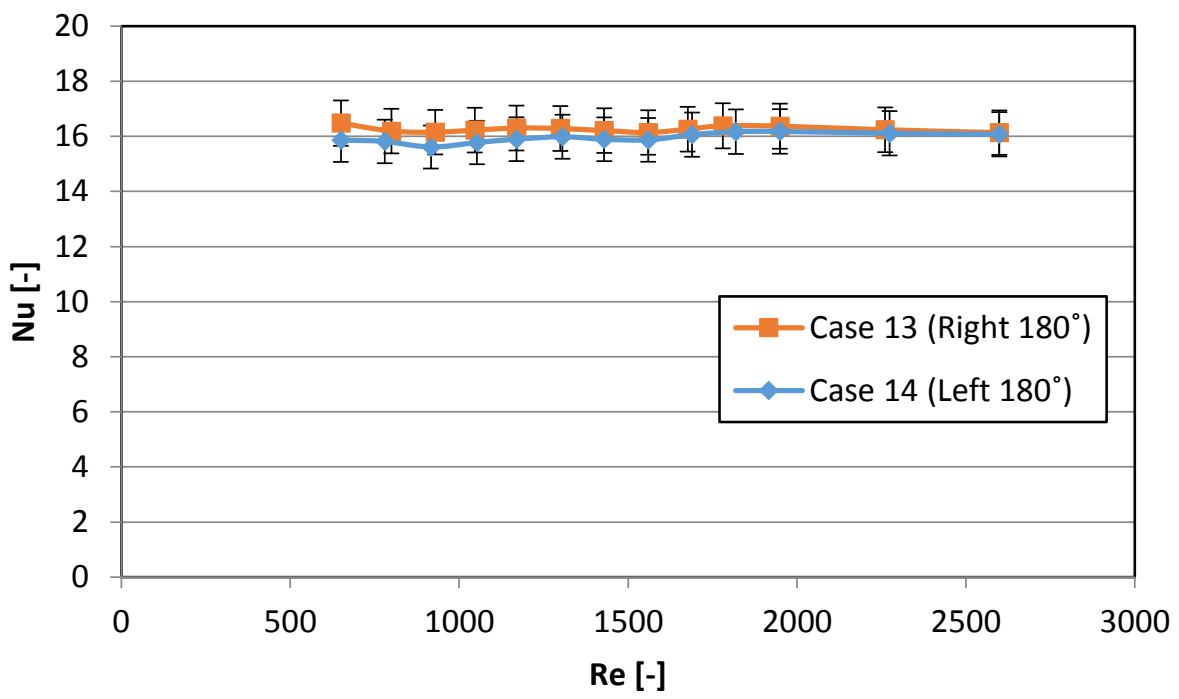

Figure 6-25: Comparison between left $180^{\circ}$ and right $180^{\circ}$ heating at $6631 \mathrm{~W} / \mathrm{m}^{2}$

\subsubsection{Influence of Heat Flux Position while keeping the Heat Flux Constant}

In this section the influence of the position of the applied heat flux distribution on the average Nusselt number is considered for cases which have the same heat flux. As mentioned earlier, the heat fluxes of interest in this study are $6631 \mathrm{~W} / \mathrm{m}^{2}, 4421 \mathrm{~W} / \mathrm{m}^{2}$ and $3316 \mathrm{~W} / \mathrm{m}^{2}$. First the Nusselt number results are presented for all cases, followed by an analysis of the Richardson number and then the correlation 
between the Nusselt number and the Richardson number is considered. It should be noted that for a given heat flux, a variation in the angle span of the applied thermal boundary condition resulted in different heat transfer rates to the water. The impact of the heat transfer rate will be considered in another section.

Figure 6-26 shows the effects of the different circumferential heating configurations for cases with a heat flux of $6631 \mathrm{~W} / \mathrm{m}^{2}$.

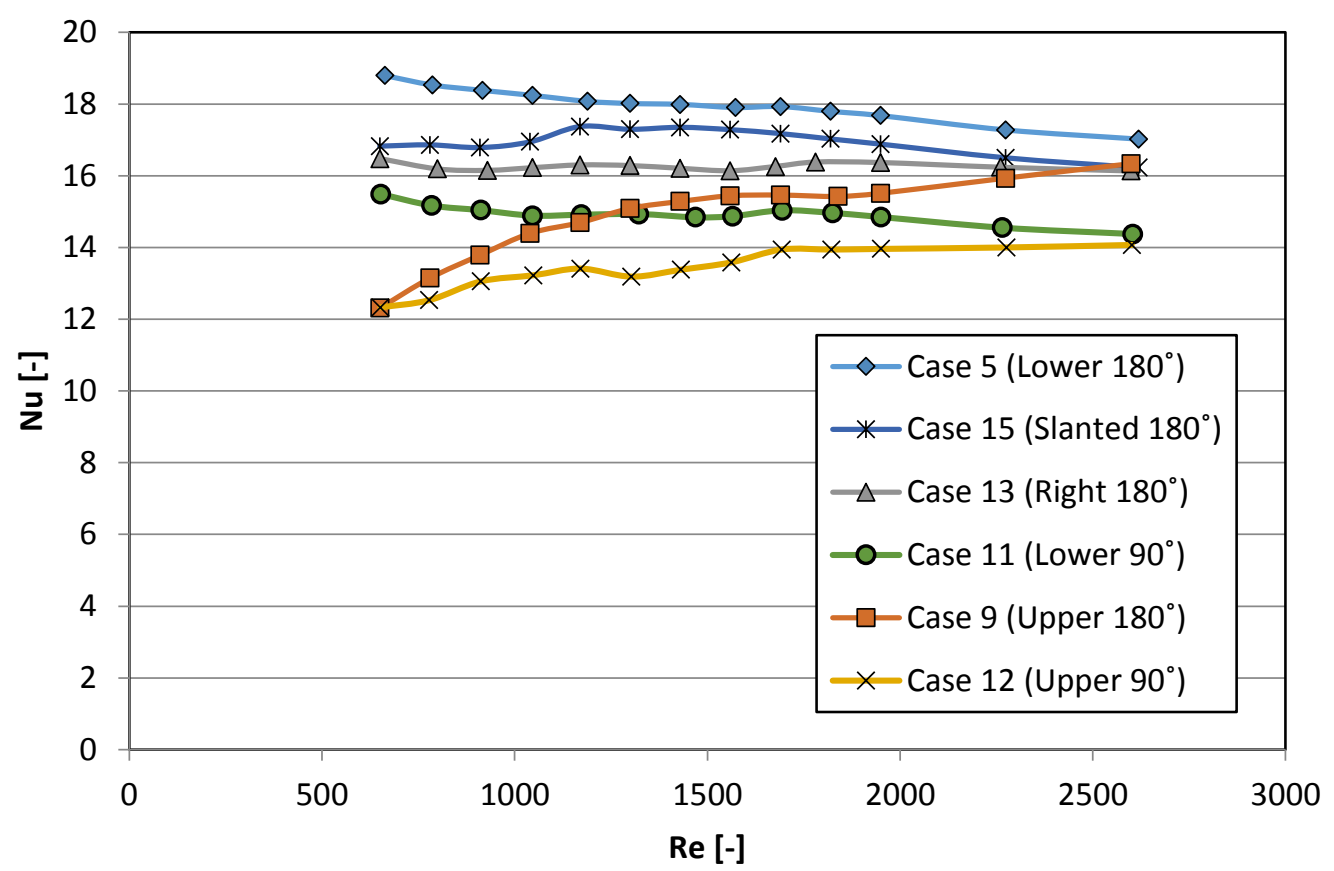

Figure 6-26: Nusselt numbers vs. Reynolds numbers at a heat flux of $6631 \mathrm{~W} / \mathrm{m}^{2}$ for different circumferential heating cases

From Figure 6-26 it is clear that the heat flux distribution has a very significant influence on the Nusselt number. At a Reynolds number of approximately 650 the difference between the best performing and worst performing cases is $41.60 \%$, while at a Reynold number of approximately 2600 , this difference is $18.9 \%$. It is seen that although the Nusselt numbers differ greatly at the lower Reynolds numbers, they do tend to converge as the Reynolds number increases.

The lower $180^{\circ}$ heating case (case 5) starts with the highest Nusselt number which can be expected due to the more prominent secondary flow created with only the lower half of the tube being heated and the large temperature profile difference for the top and bottom of the tube. More information on the effects of the buoyancy driven secondary flow is given when the Richardson number analysis is presented later in this section.

The same pattern is seen for the slanted $180^{\circ}$ heating case (case 15), although not as pronounced. The right side $180^{\circ}$ heating case (case 13 ) which is similar to the left side $180^{\circ}$ heating case, exhibit a relatively constant Nusselt number throughout the laminar flow regime. The Nusselt numbers for the upper $180^{\circ}$ heating case (case 8) increases sharply with an increase in the Reynolds number after starting at a relatively low value. The effect of the different heating configurations has on the Nusselt numbers is very evident in the above graph and clearly shows the influence secondary flow has on the heat transfer. 
Figure 6-27 shows the Richardson numbers for the cases in Figure 6-26. For convenience $\mathrm{Ri}=10$ is labelled to identify which of the data points fall in the mixed convection range (according to the Richardson guidelines mentioned earlier). The large Richardson numbers at the lower Reynolds numbers indicate that the means of heat transfer at these Reynolds numbers is mostly via natural convection. Figure 6-27 also shows that as the Reynolds numbers increase forced convection becomes more prevalent in all the cases. The upper heating cases have significantly lower starting Richardson numbers when compared to the other cases with similar heat inputs. Therefore, it can be seen that there is less secondary flow in these cases (as stated earlier). All the cases end with very similar Richardson numbers showing that the amount of secondary flow present in the cases is comparable.

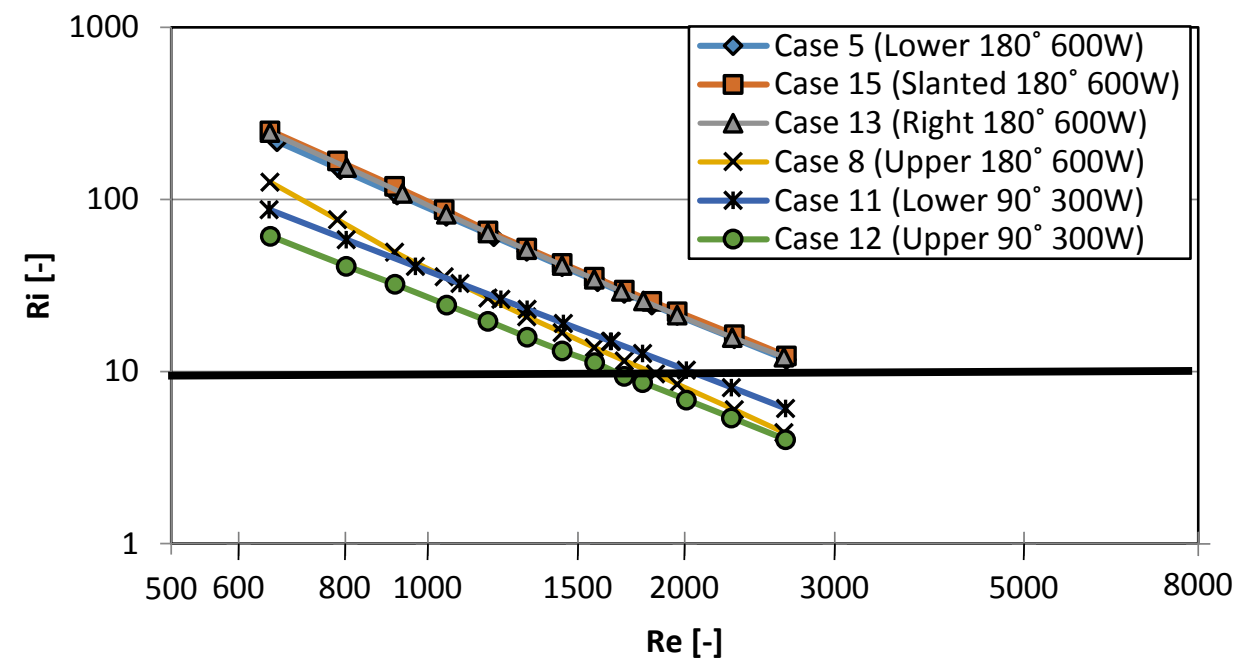

Figure 6-27: Richardson numbers vs. Reynolds numbers at a heat flux of $6631 \mathrm{~W} / \mathrm{m}^{2}$ for different circumferential heating cases

Figure 6-28 shows the results for an applied heat flux of $4421 \mathrm{~W} / \mathrm{m}^{2}$. It includes a $360^{\circ}$ heating case, which can be used as a benchmark for the other heating cases. The Nusselt number results for the $360^{\circ}$ case remains fairly constant over the full range of Reynolds numbers tested. As seen before the lower $180^{\circ}$ heating case starts higher than its upper $180^{\circ}$ counterpart. This is once again due to the secondary flow aiding in the heat transfer in the flow. It is once again seen that the two cases converge quite clearly when nearing the turbulent flow regime. Both the $180^{\circ}$ cases are however seen to produce lower Nusselt numbers when compared to the $360^{\circ}$ case. 


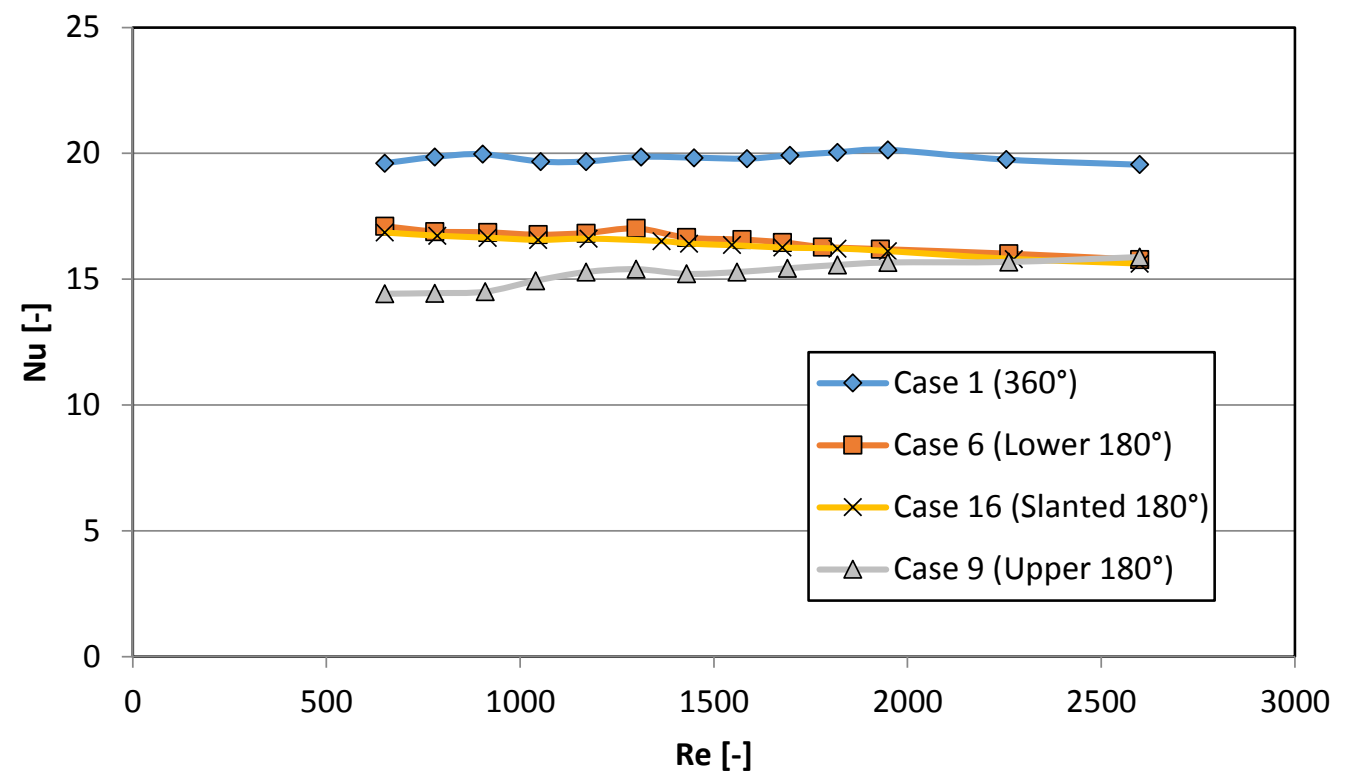

Figure 6-28: Nusselt numbers vs. Reynolds numbers at a heat flux of $4421 \mathrm{~W} / \mathrm{m}^{2}$ for different circumferential heating cases

Figure 6-28 shows the $180^{\circ}$ heating cases with the context of the $360^{\circ}$ case, which was not shown in Figure 6-26 above. This shows that the $360^{\circ}$ heating case still provides higher Nusselt numbers when compared to the circumferentially non-uniform cases. This can be explained by the fact that the $360^{\circ}$ cases have more heat input than the other cases. The impact of the heat flux is shown in the next section.

Figure 6-29 shows that the cases with higher Nusselt numbers also have higher Richardson numbers. The secondary flow and mixed convection present in the flow is once again given as the reason for this correlation.

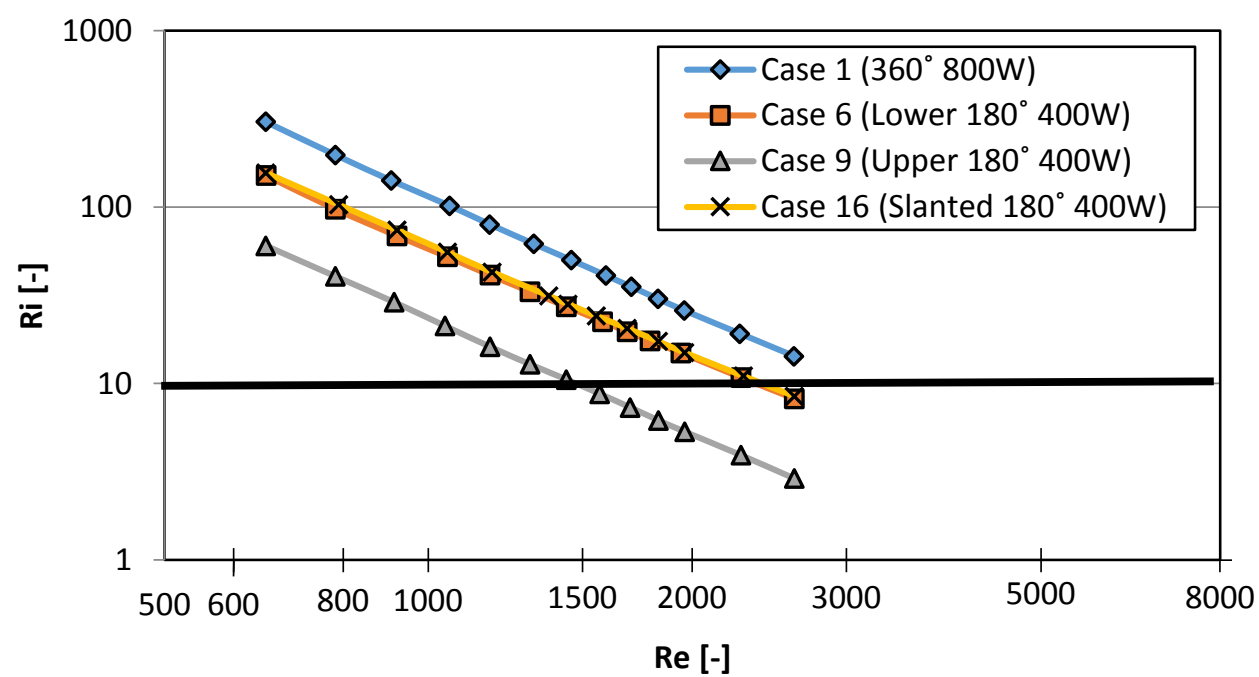

Figure 6-29: Richardson numbers vs. Reynolds numbers at a heat flux of $4421 \mathrm{~W} / \mathrm{m}^{2}$ for different circumferential heating cases 
Figure 6-30 shows the results for an applied heat flux of $3316 \mathrm{~W} / \mathrm{m}^{2}$. It is very similar to Figure 6-28 with the cases showing very similar trends and the Nusselt numbers being very similar, in range, between the two graphs. The upper and lower $180^{\circ}$ cases Nusselt numbers are seen to converge earlier than in the $4421 \mathrm{~W} / \mathrm{m}^{2}$ cases.

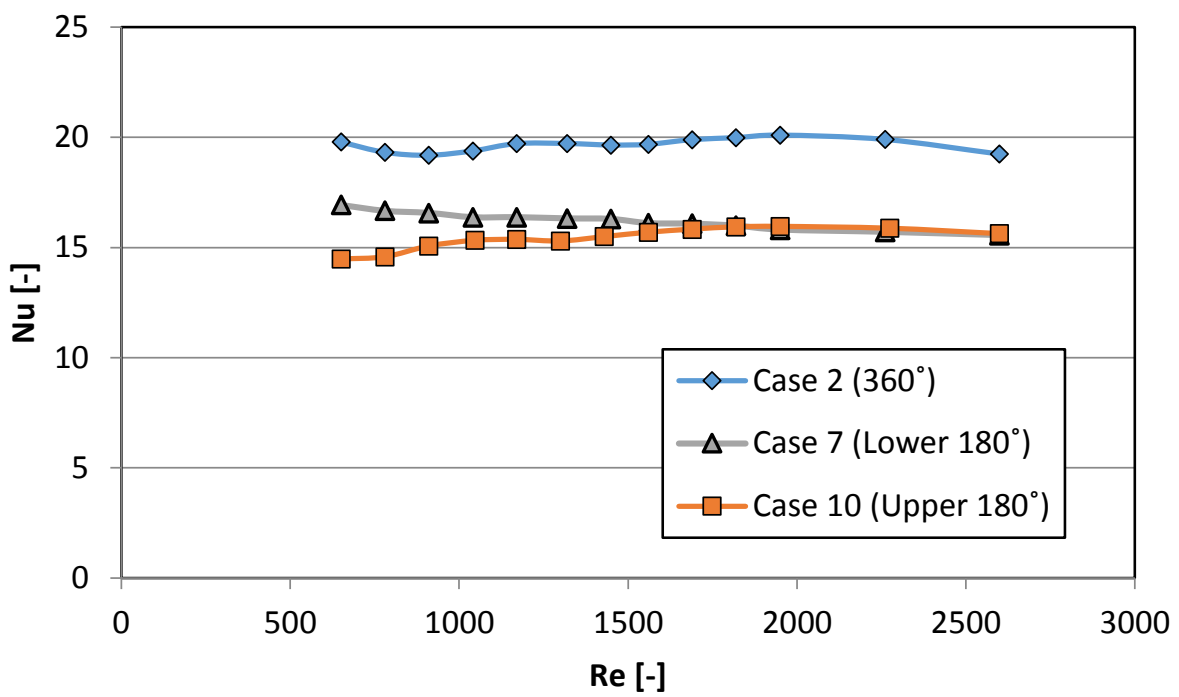

Figure 6-30: Nusselt numbers vs. Reynolds numbers at a heat flux of $3316 \mathrm{~W} / \mathrm{m}^{2}$ for different circumferential heating cases

Figure 6-31 once again shows the reason for the discrepancies between the upper and lower heating cases. The clear difference in the Richardson numbers shows the difference in the secondary flow present in each case.

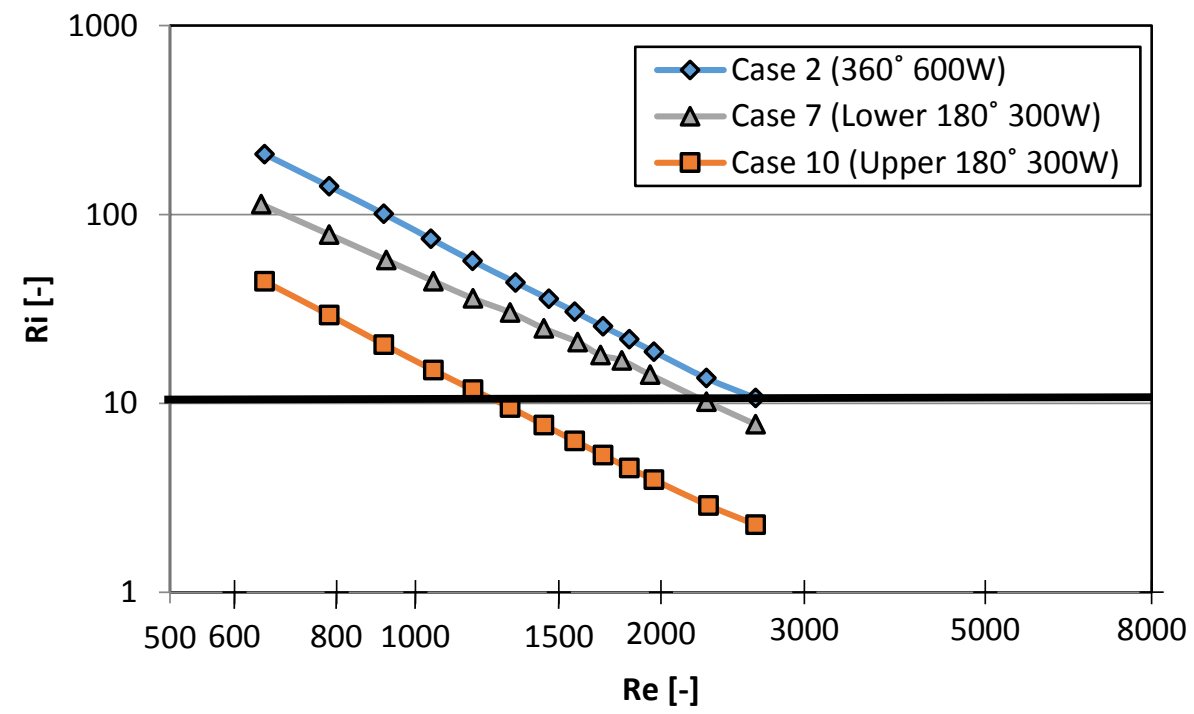

Figure 6-31: Richardson numbers vs. Reynolds numbers at a heat flux of $3316 \mathrm{~W} / \mathrm{m}^{2}$ for different circumferential heating cases

\subsubsection{Influence of Heat Flux Position while keeping the Heat Transfer Rate Constant}

In the previous section the influence of the heat flux distribution was presented while maintaining a constant heat flux on the heated perimeter segment of the pipe wall. Thus, as mentioned, a variation 
in the heated angular span resulted in different heat transfer rates. In this section the influence of the heated angular spans on the average heat transfer coefficient is considered for cases which had the same heat transfer rate to the water. The heat transfer rates of interest in this section are 600W, $400 \mathrm{~W}$ and $300 \mathrm{~W}$. It should be noted that maintaining a particular heat transfer rate across various angular spans of heating, required different localized heat fluxes. The results presented in this section must thus be considered with the results in the previous section in order to better understand what the influences are of $(a)$ the angle span, (b) the localized heat flux and (c) the overall heat transfer rate to the water.

Figure 6-32 presents the results of the cases where the heat transfer rate was $600 \mathrm{~W}$.

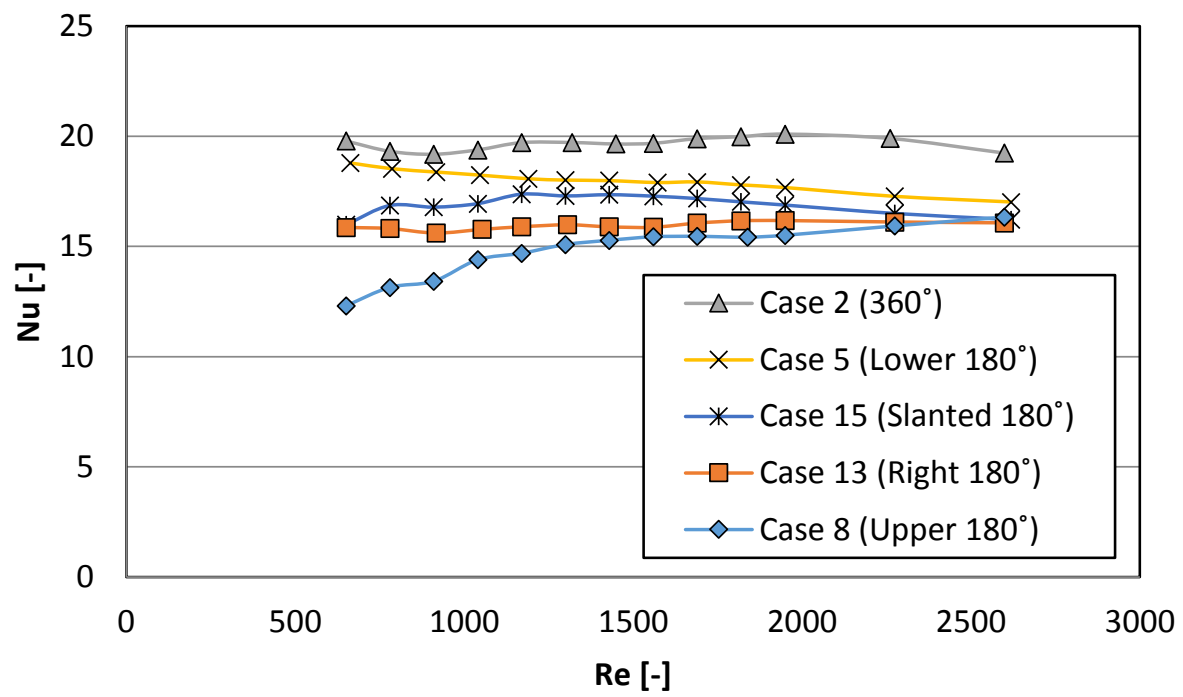

Figure 6-32: Nusselt number vs. Reynolds number for different circumferential heating configurations at the identical total heat input of $600 \mathrm{~W}$

Figure 6-33 shows the Richardson numbers associated with Figure 6-32. The Richardson numbers for case 2 , case 5, case 13 and case 15 are very similar, with the Richardson number for the upper $180^{\circ}$ heating case (case 8 ) being substantially lower, as expected. 


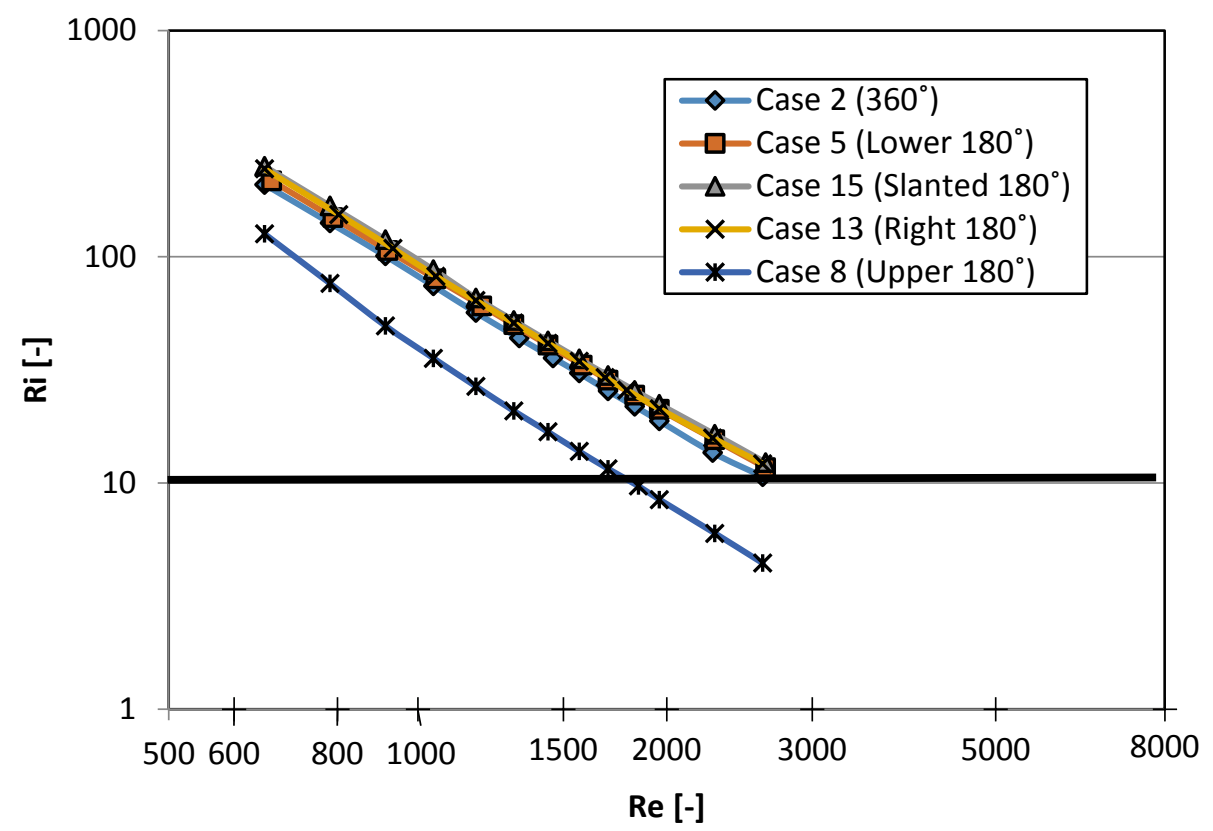

Figure 6-33: Richardson number vs. Reynolds number for different circumferential heating configurations at the identical total heat input of $600 \mathrm{~W}$

Figure 6-34 shows the Nusselt numbers for cases of different angular heating spans with a heat transfer rate of $400 \mathrm{~W}$. The $360^{\circ}$ heating case (case 3) performs best once again, with the lower and slanted heating cases performing very similarly. The upper heating case shows the lowest Nusselt number as expected.

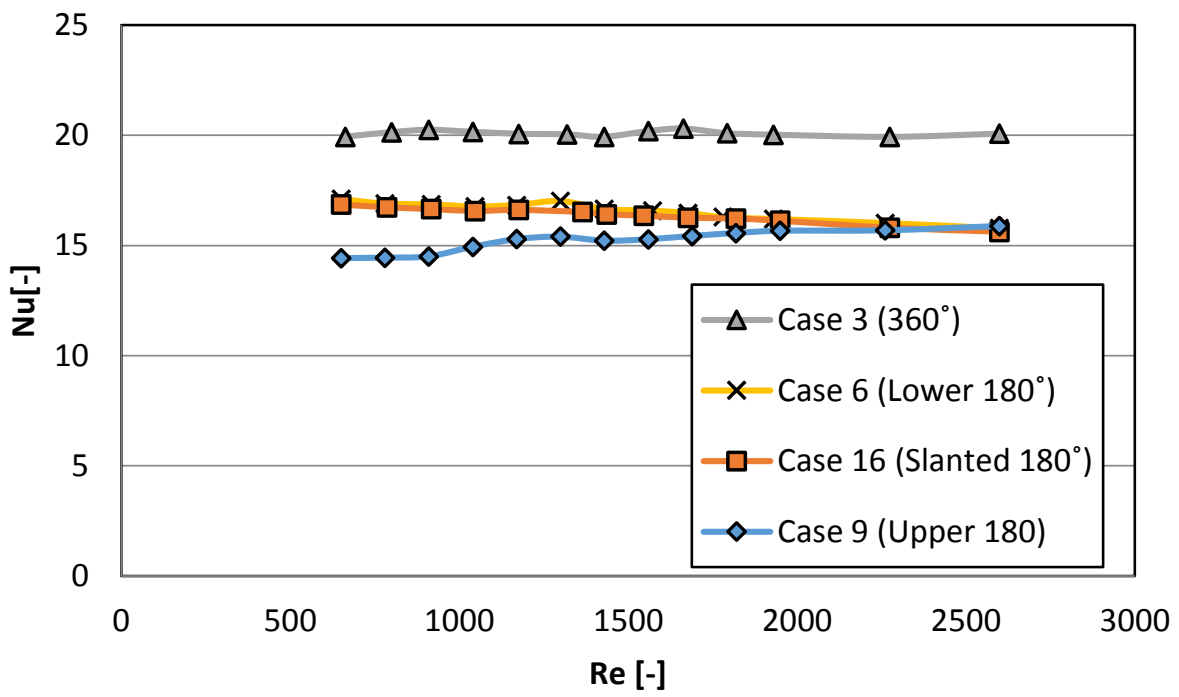

Figure 6-34: Nusselt number vs. Reynolds number for different circumferential heating configurations at the identical total heat input of $400 \mathrm{~W}$

Figure 6-35 is very similar to Figure 6-33 as it shows that Richards numbers for all the cases are almost the same, baring the upper $180^{\circ}$ heating case. 


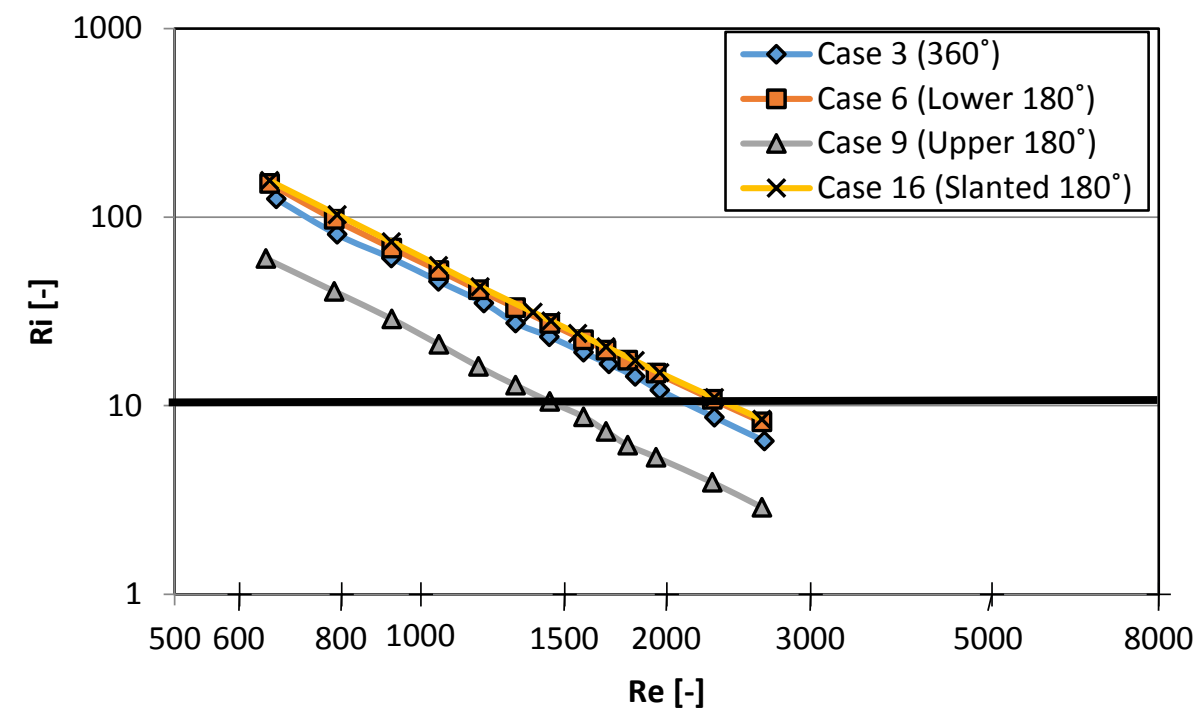

Figure 6-35: Richardson number vs. Reynolds number for different circumferential heating configurations at the identical total heat input of $400 \mathrm{~W}$

Figure 6-36 reiterates that the $360^{\circ}$ heating case gives the highest Nusselt numbers. Case 7, case 10 and case 11 are very similar throughout the Reynolds number range. Case 12 (upper $90^{\circ}$ ) performs poorly yet converges with case 11 (lower $90^{\circ}$ ) at higher Reynolds numbers.

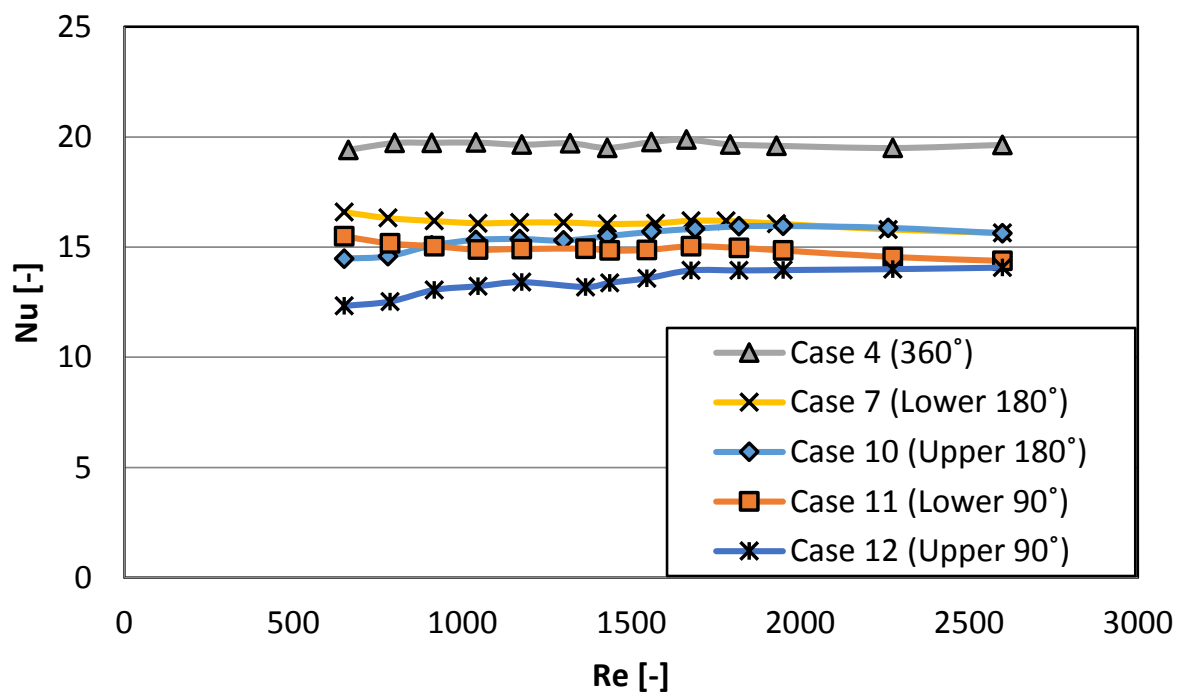

Figure 6-36: Nusselt number vs. Reynolds number for different circumferential heating configurations at the identical total heat input of $300 \mathrm{~W}$

Figure 6-37 shows that case 7 (lower $180^{\circ}$ ) has the highest Richardson number at lower Reynolds numbers which is expected. However, case 11 and case $12\left(90^{\circ}\right.$ heating cases) having higher Richardson numbers than case 10 (upper $180^{\circ}$ ) is interesting. 


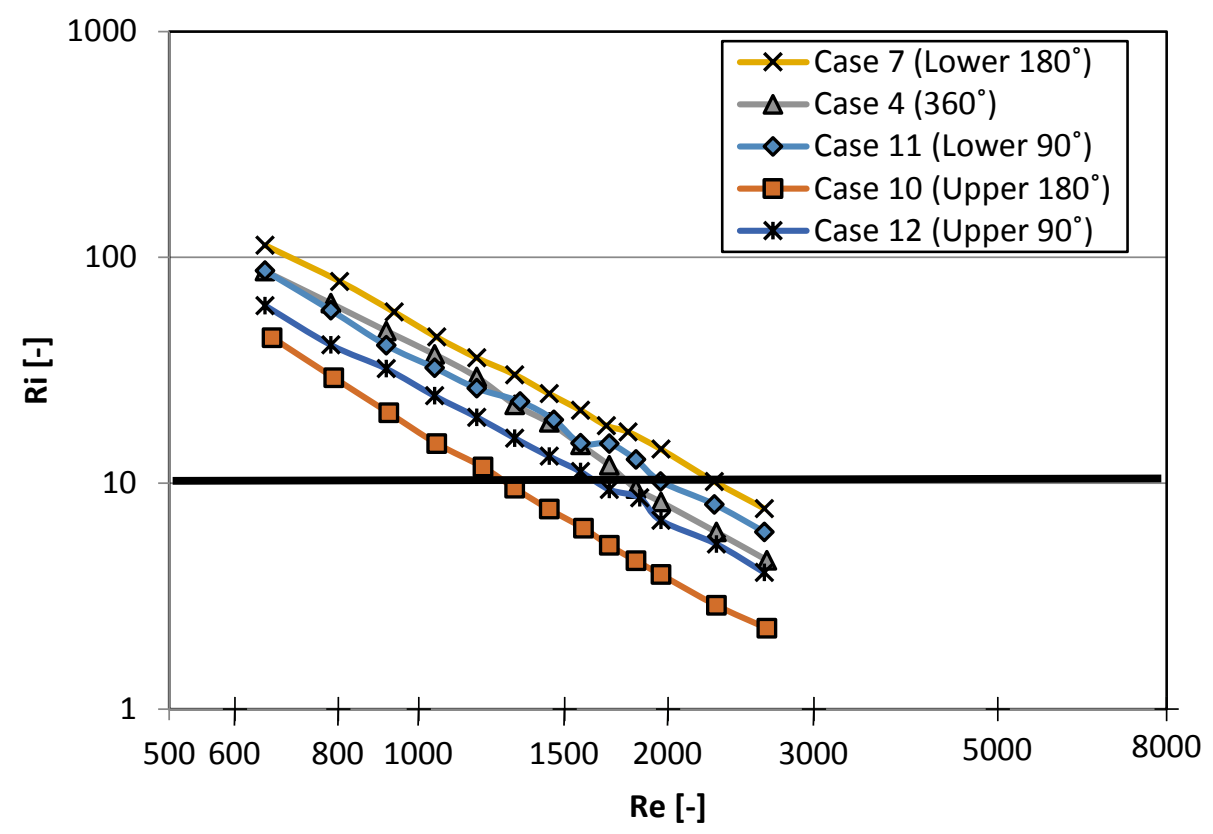

Figure 6-37: Richardson number vs. Reynolds number for different circumferential heating configurations at the identical total heat input of $300 \mathrm{~W}$

\subsubsection{Impact of Heat Flux}

The graphs in this section show the effect of the change of heat flux on the same heating configuration. This is done for the $360^{\circ}$, lower $180^{\circ}$ and upper $180^{\circ}$ heating cases.

Figure 6-38 shows that the Nusselt numbers are virtually unaffected by the change in heat flux for cases with a fully uniform heat flux distribution. With a maximum difference of $2 \%$ between the cases which falls within the uncertainty of the average Nusselt number.

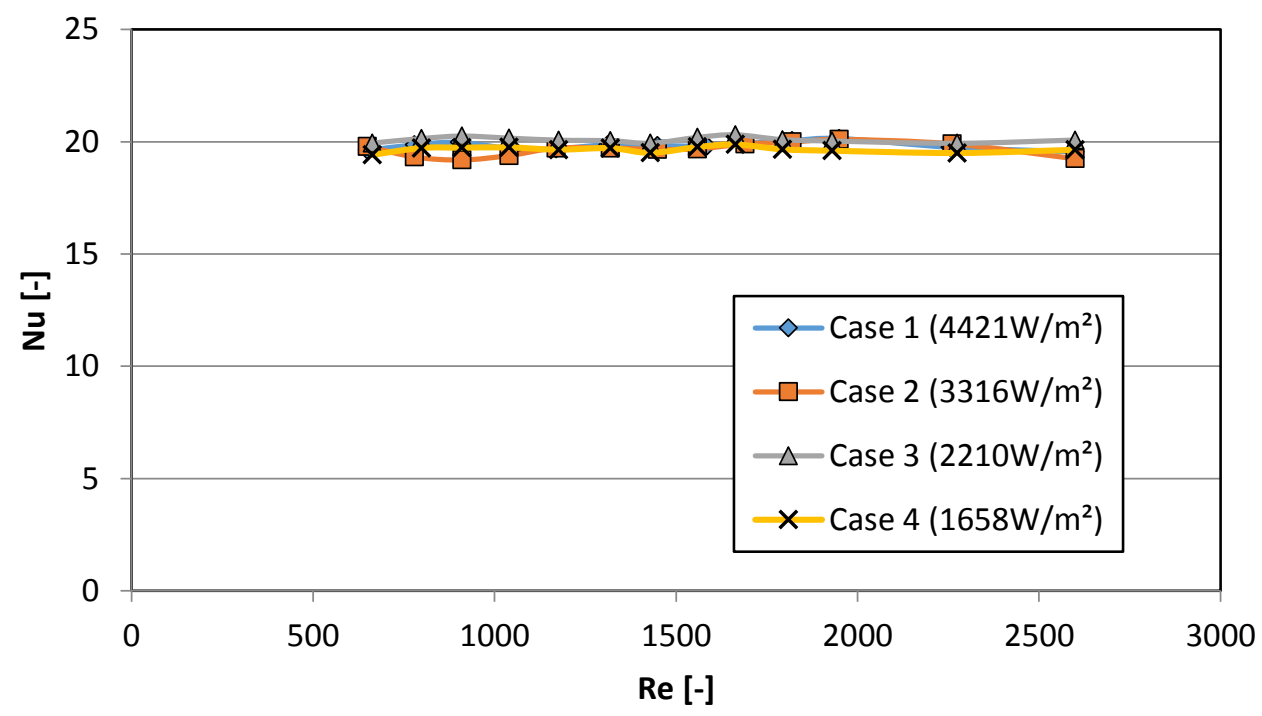

Figure 6-38: Nusselt numbers vs. Reynolds numbers for $360^{\circ}$ heating cases at different heat flux's

Figure 6-39 shows the Richardson numbers for cases 1-4. The Richardson numbers decrease as the heat flux decreases. This is most prominently seen at lower Reynolds numbers. With the Richardson numbers converging at higher Reynolds numbers. 


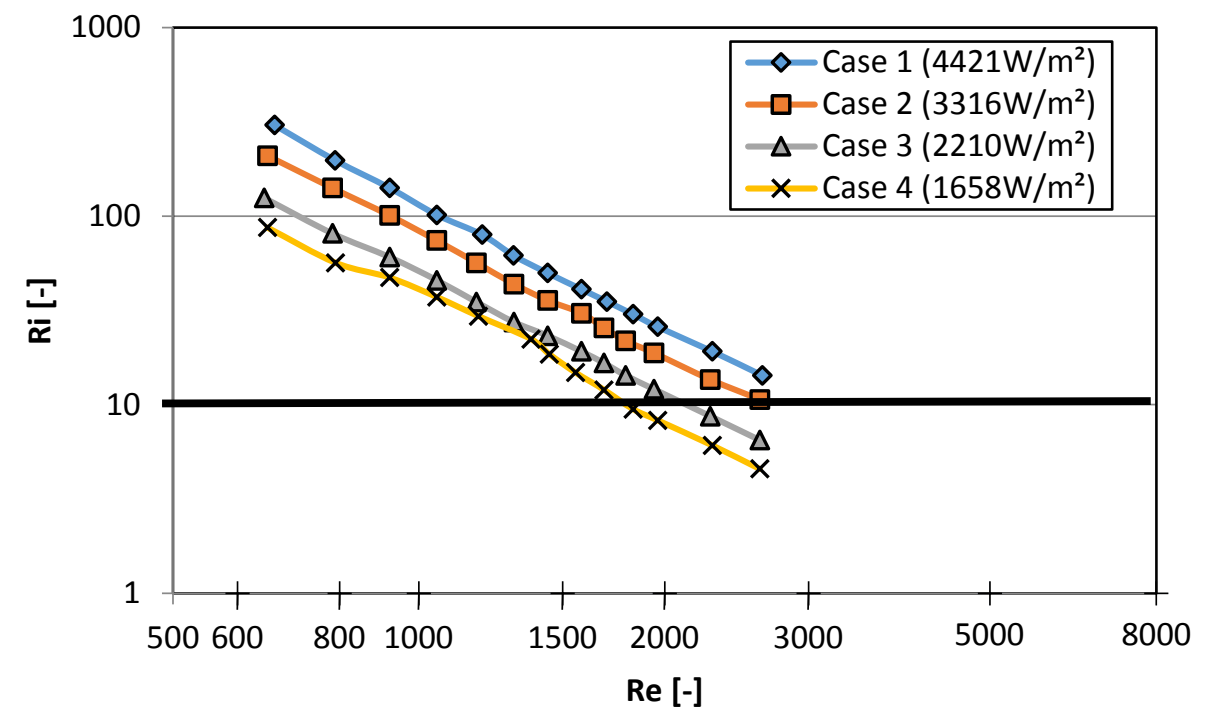

Figure 6-39: Richardson numbers vs. Reynolds numbers for $360^{\circ}$ heating cases at different heat flux's

Figure 6-40, unlike Figure 6-38, shows a difference in the Nusselt numbers for the different heat fluxes distributed on the lower half of the tube. The reason for case 5 showing a different (higher) Nusselt number could be due to the exaggerated secondary flow induced by the heating being concentrated to the lower half of the tube. There is an overall tendency for Nusselt numbers from lower $180^{\circ}$ heating cases to decrease as the Reynolds number increases. This is once again due to the decrease in secondary flow as the main flow speeds up.

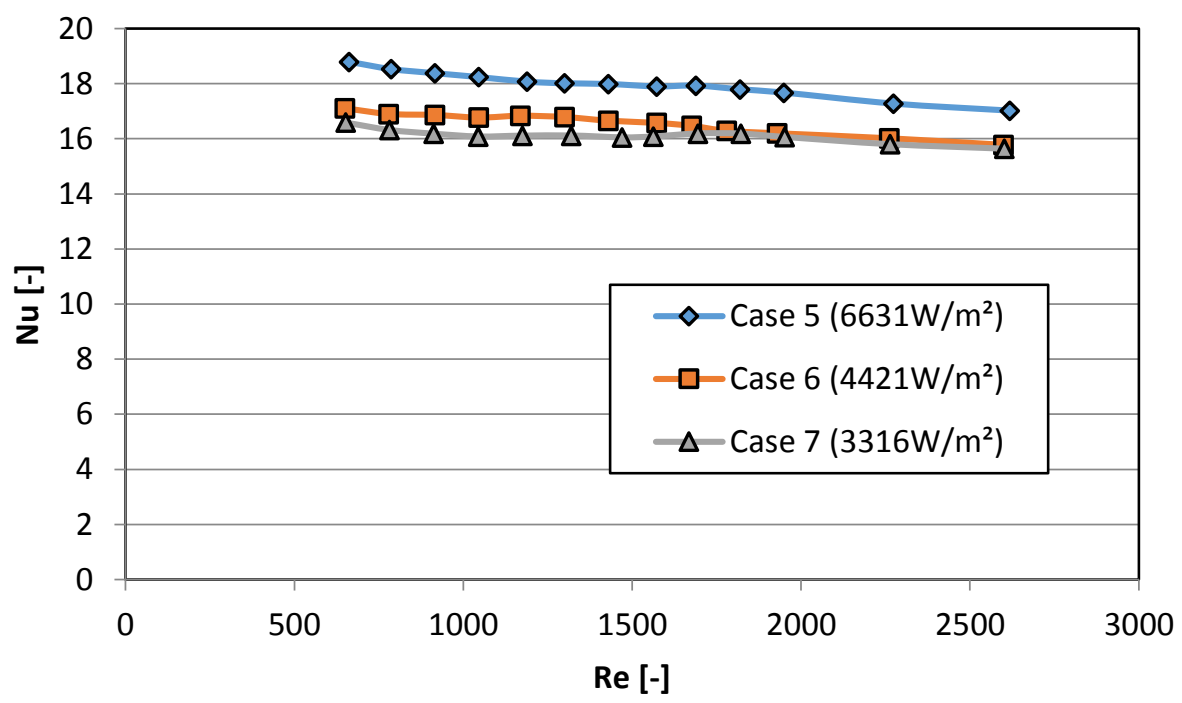

Figure 6-40: Nusselt numbers vs. Reynolds numbers for lower $180^{\circ}$ heating cases at different heat flux's

Although Figure 6-41 shows that the Richardson numbers for case 5 are higher than the other cases. A larger discrepancy would have been preferred to ensure the reason for the higher Nusselt numbers for case 5 in Figure 6-40 being due to there being a higher secondary flow being present. It is thus possible, based on these results that the Richardson number is not perfectly suitable to explain the difference in the Nusselt number fully. 


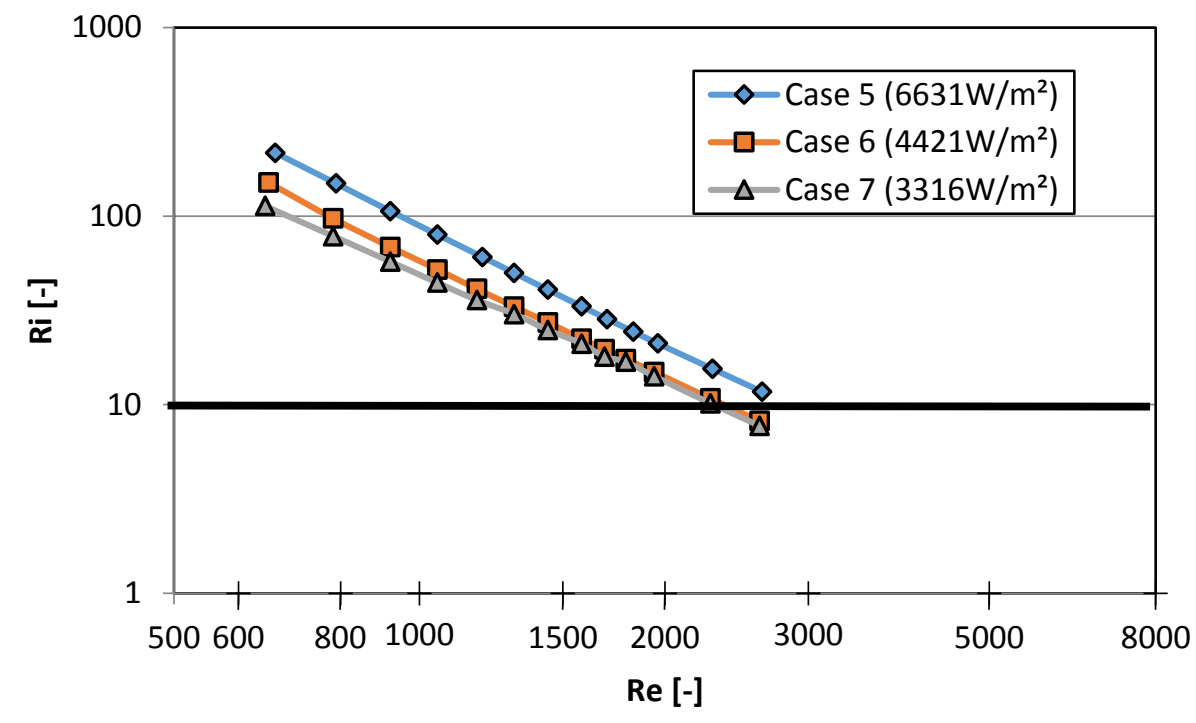

Figure 6-41: Richardson numbers vs. Reynolds numbers for lower $180^{\circ}$ heating cases at different heat flux's

From Figure 6-42 it is seen that for cases with upper half heating that the Nusselt numbers are relatively unaffected by a change in the heat flux, with a maximum difference of $5 \%$ for the entire range. It can also be seen that there is an overall tendency for Nusselt numbers from upper $180^{\circ}$ heating cases to increase as the Reynolds number increases.

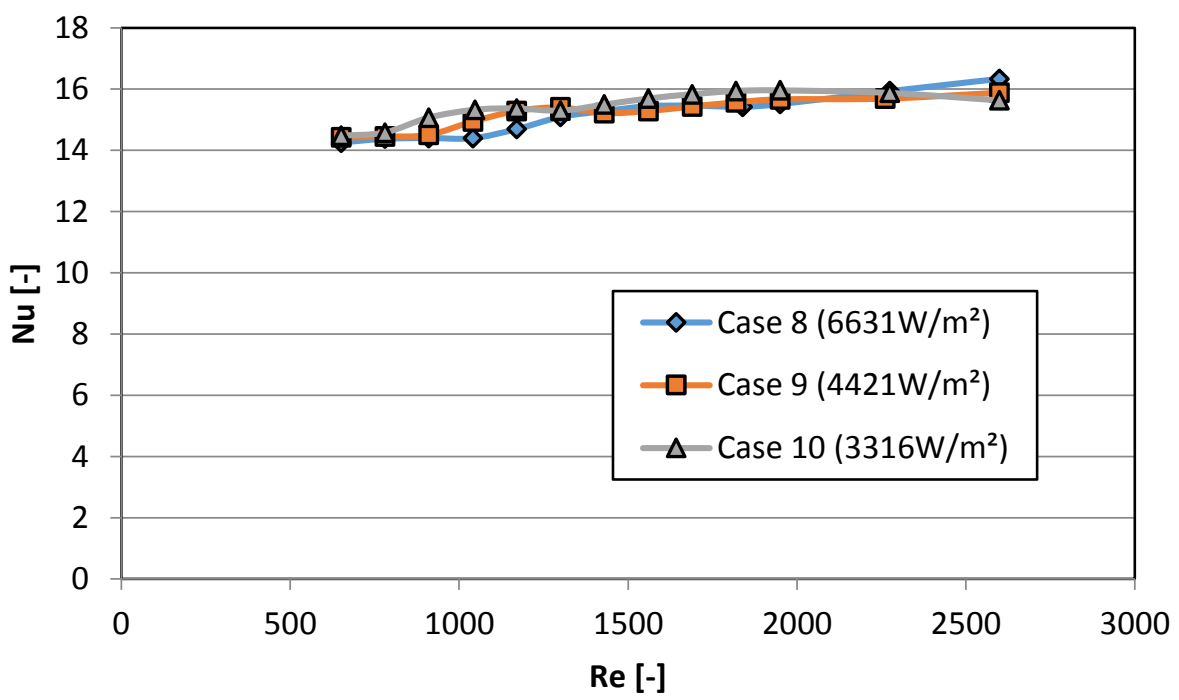

Figure 6-42: Nusselt numbers vs. Reynolds numbers for upper $180^{\circ}$ heating cases at different heat flux's

Figure 6-43 shows the Richardson number for the upper $180^{\circ}$ heating cases. A trend very similar to the rest of the Richardson number plots is visible. The higher the heat flux the higher the Richardson number. This is, once again, more pronounced at lower Reynolds numbers, as expected. 


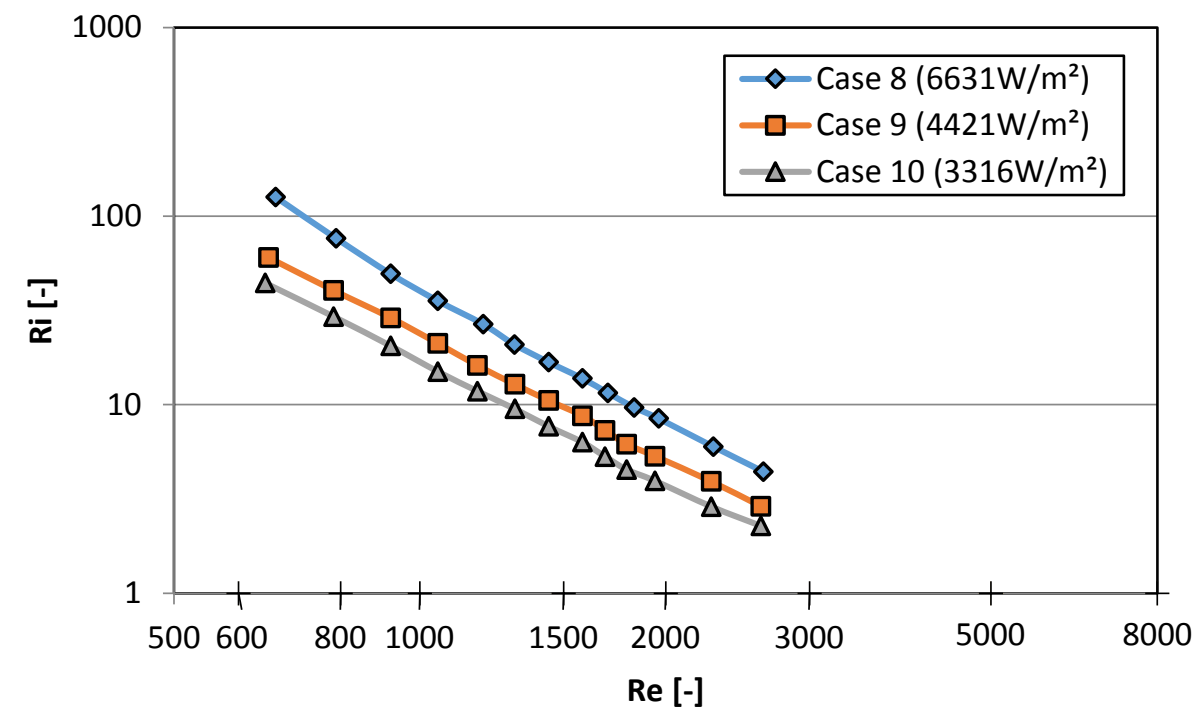

Figure 6-43: Richardson numbers vs. Reynolds numbers for upper $180^{\circ}$ heating cases at different heat flux's

\subsection{Effect of the Richardson Number on Nusselt Numbers}

To better understand the effect the Richardson number has on the Nusselt numbers obtained experimentally, a number of different graphs are plotted in this section.

Figure 6-44 shows the effect that the Richardson number has on the Nusselt number for the $360^{\circ}$ heating cases (containing all the tested heat fluxes), the lower $180^{\circ}$ heating cases (all tested heat fluxes) and the upper $180^{\circ}$ cases (all tested heat fluxes) at a Reynolds number of 700 . The lines added to each data series are simply to better illustrate the trend of the data. The $360^{\circ}$ cases remain fairly constant irrespective of the change in Richardson number. The lower $180^{\circ}$ heating case shows that there is an increase in the Nusselt number as the Richardson number is increased. The opposite is observed for the upper $180^{\circ}$ heating cases. The increase in Richardson number decreases the Nusselt number for these cases.

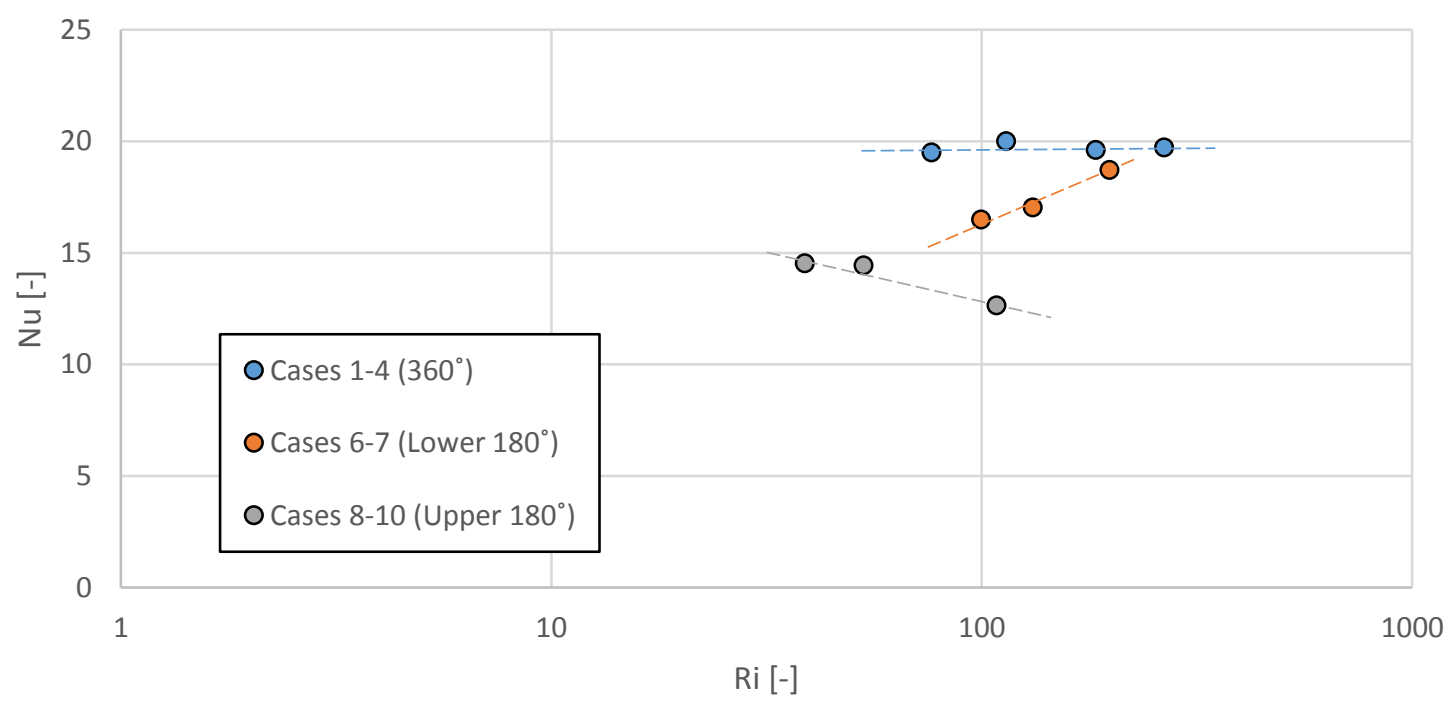

Figure 6-44: Nusselt number vs Richardson number for $360^{\circ}$, lower $180^{\circ}$ and upper $180^{\circ}$ heating at $\operatorname{Re}=700$ 
Figure 6-45 shows the effect of the Richardson number on the Nusselt number for the $360^{\circ}$ heating cases (all heat fluxes tested) at different Reynolds numbers. It is evident from the figure that although the Reynolds number affects the Richardson number quite severely (the higher the Reynolds number the lower the Richardson number), neither the Reynolds number nor the Richardson number have any significant effect on the average Nusselt number for $360^{\circ}$ heating cases.

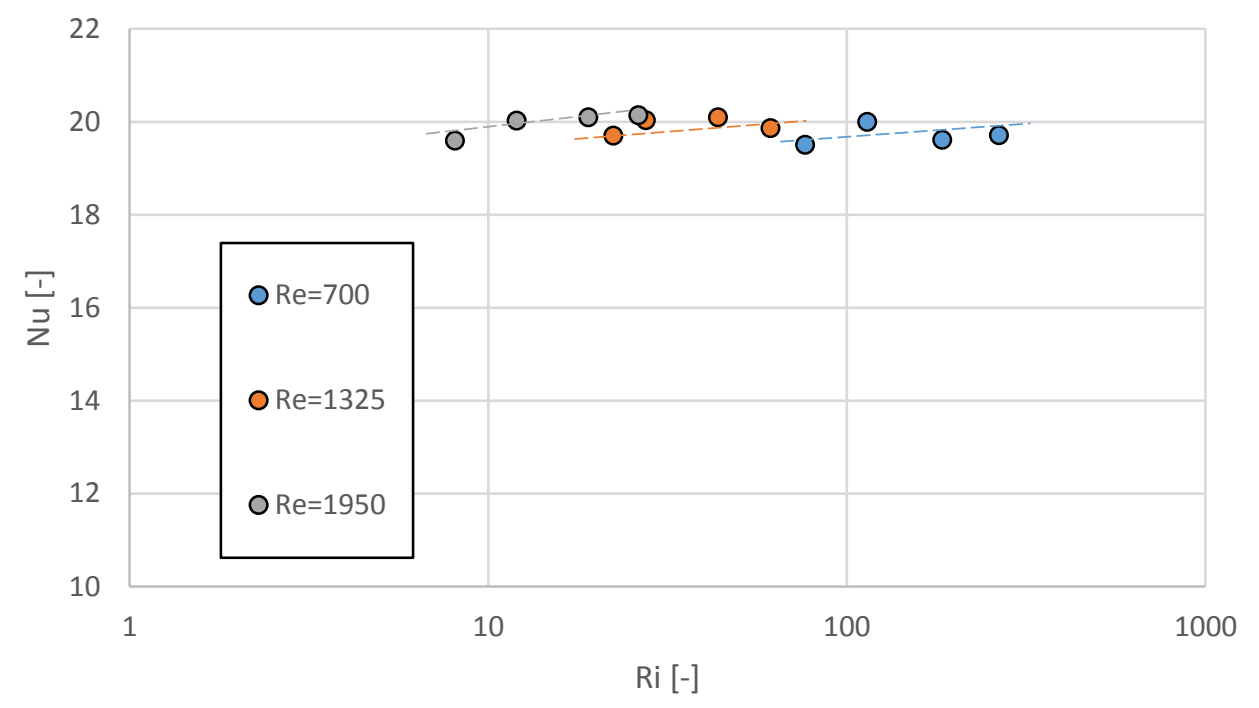

Figure 6-45: Nusselt numbers vs Richardson numbers for the $360^{\circ}$ heating cases at different Reynolds numbers

Figure 6-46 shows the effect of the Richardson number on the Nusselt number for the lower $180^{\circ}$ heating cases (all heat fluxes tested) at different Reynolds numbers. Once again it is clear the Richardson number decreases as the Reynolds number is increased. However, unlike Figure 6-45, the Nusselt numbers slightly decrease with the increase of the Reynolds number. This is consistent with what has been observed in this study.

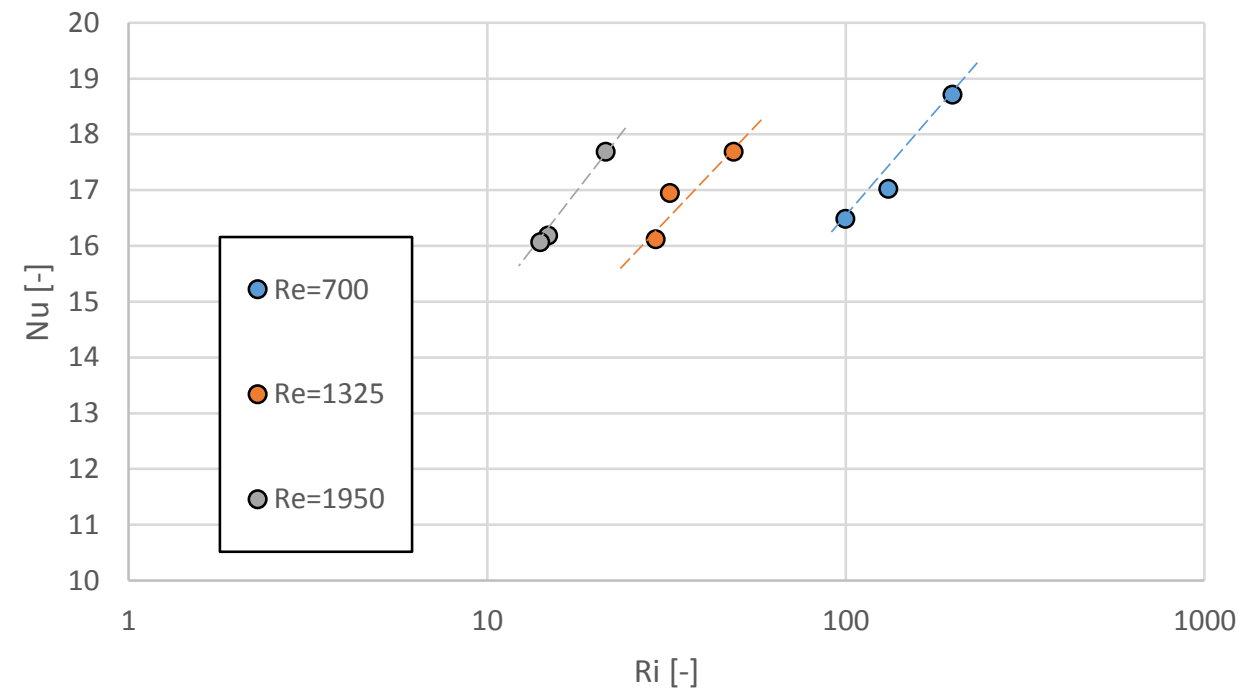

Figure 6-46: Nusselt numbers vs Richardson numbers for the lower $180^{\circ}$ heating cases at different Reynolds numbers 
Figure 6-47 shows the effect of the Richardson number on the Nusselt number for the upper $180^{\circ}$ heating cases (all heat fluxes tested) at different Reynolds numbers. The graph shows that the Richardson number decreases while the Reynolds number increases. But the opposite is true for the Nusselt number, as it is seen to rise with the Reynolds number increase.

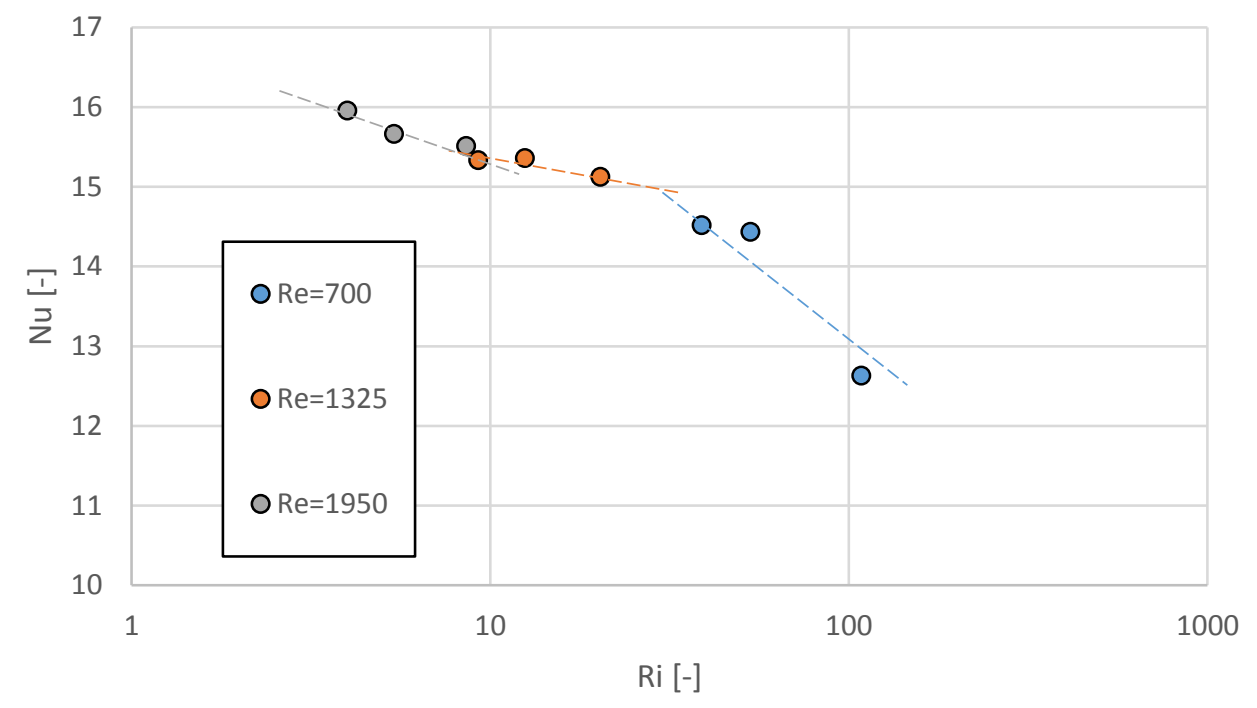

Figure 6-47: Nusselt numbers vs Richardson numbers for the upper $180^{\circ}$ heating cases at different Reynolds numbers

\subsection{Effect of Heating Position on Richardson Number}

With so many different heating configurations being tested, the opportunity exists to investigate the effect the position of the heat flux has on the Richardson and Nusselt numbers. Figure 6-48 shows the cross section of the test section pipe with different angles of Theta displayed. Each displayed angle is the centre point of heating for one of the $6631 \mathrm{~W} / \mathrm{m}^{2} 180^{\circ}$ heating cases. $0^{\circ}$ is the centre of the upper $180^{\circ}$ heating case, $90^{\circ}$ is the centre of the right $180^{\circ}$ heating case, $135^{\circ}$ is the centre of the slanted heating case and $180^{\circ}$ is the centre of the lower $180^{\circ}$ heating case. 


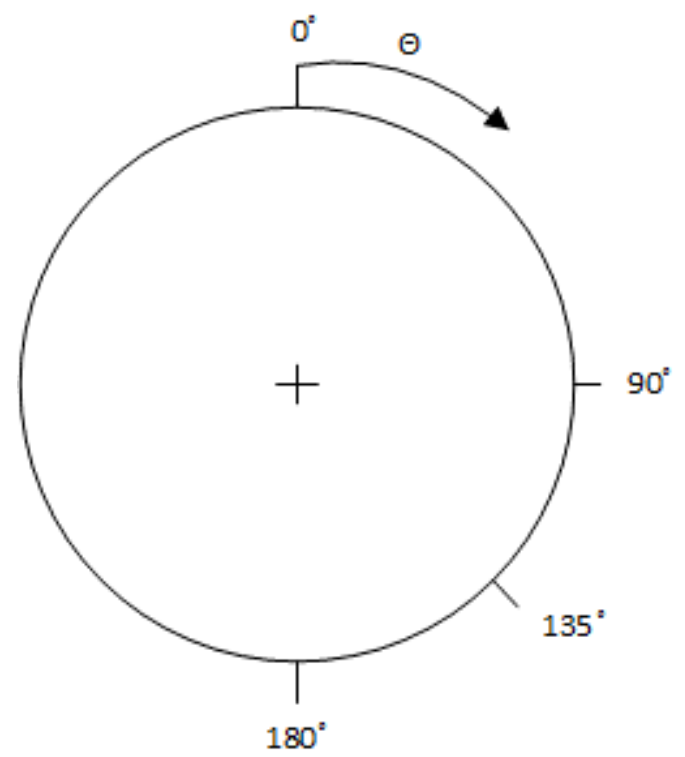

Figure 6-48: Schematic showing the different degrees of the pipe

Figure 6-49 shows that the highest Richardson numbers are observed if the heating is being concentrated either on the right side or slanted. The lower heating is slightly below the right and slanted heating cases. The upper half heating has a significantly lower Richardson number when compared to the other cases. This is expected due to the lack of buoyancy forces for upper half heated cases.

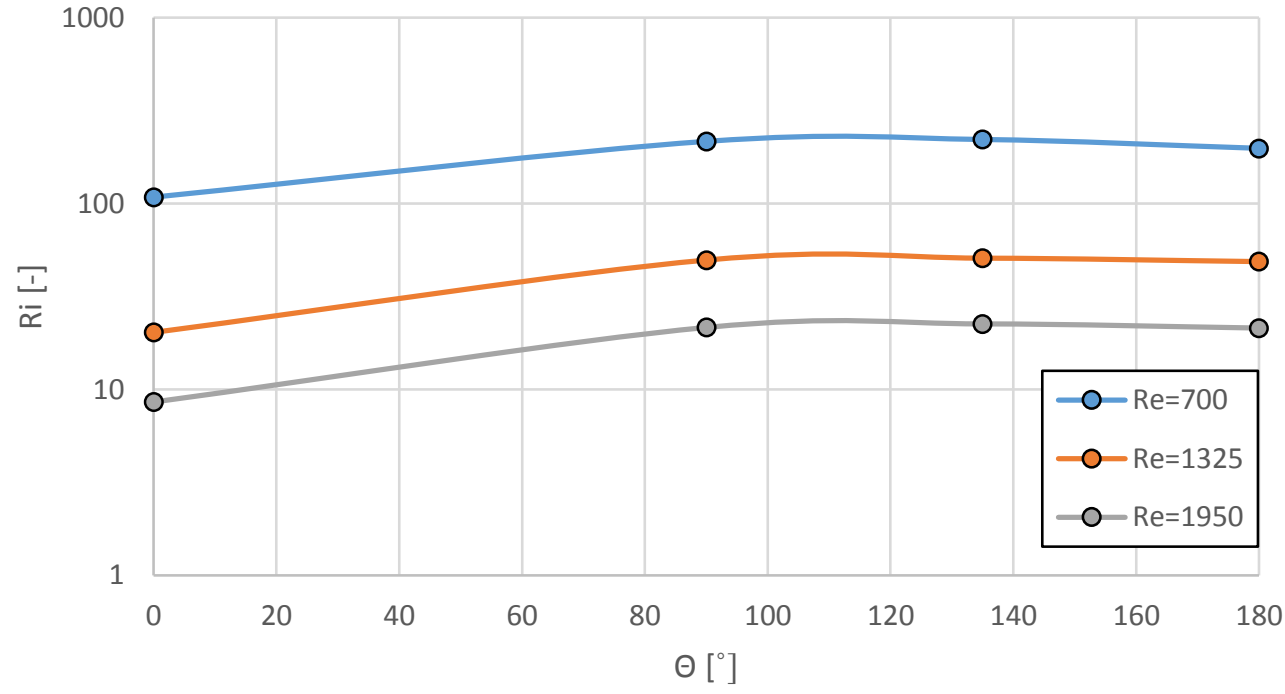

Figure 6-49: Richardson number vs $\Theta$ at different Reynolds numbers

Figure 6-50 shows the highest Nusselt numbers are seen when the heating is concentrated at $180^{\circ}$. The heating concentrated at $0^{\circ}$ shows the lowest Nusselt numbers for all the Reynolds numbers tested. A trend of the Nusselt number increasing as the heat is concentrated at higher angles is observed ( $180^{\circ}$ being the highest as the left and right hemispheres are symmetrical). The trend is most plainly obvious in the lower Reynolds numbers. 


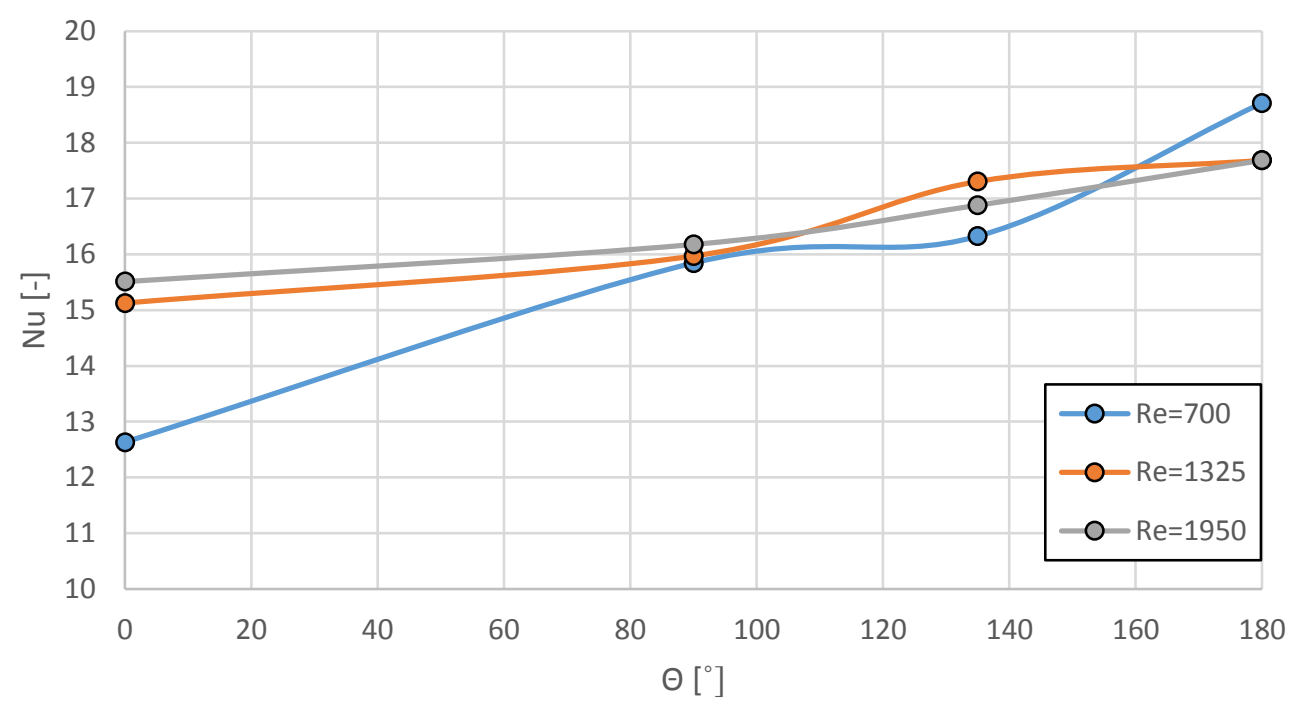

Figure 6-50: Nusselt number vs $\Theta$ at different Reynolds numbers

\subsection{Correlations}

Once all the data is recorded and interpreted correlations can be formed. Three different correlations will be presented in this section. The first correlation will be for determining the Nusselt number for a normal uniform $\left(360^{\circ}\right)$ heating case. The second correlation is for calculating the Nusselt number for lower $180^{\circ}$ heating cases. And the final correlation will be for determining the Nusselt number for upper $180^{\circ}$ heating cases.

Figure 6-51 shows data points for all the cases investigated in this section. The data points are seen to be widely spread with other factors obviously playing a part on the Nusselt number versus Richardson number relationship. From the previous sections, it is clear that the Reynolds number plays a large role in both the Nusselt number and more prominently the Richardson number.

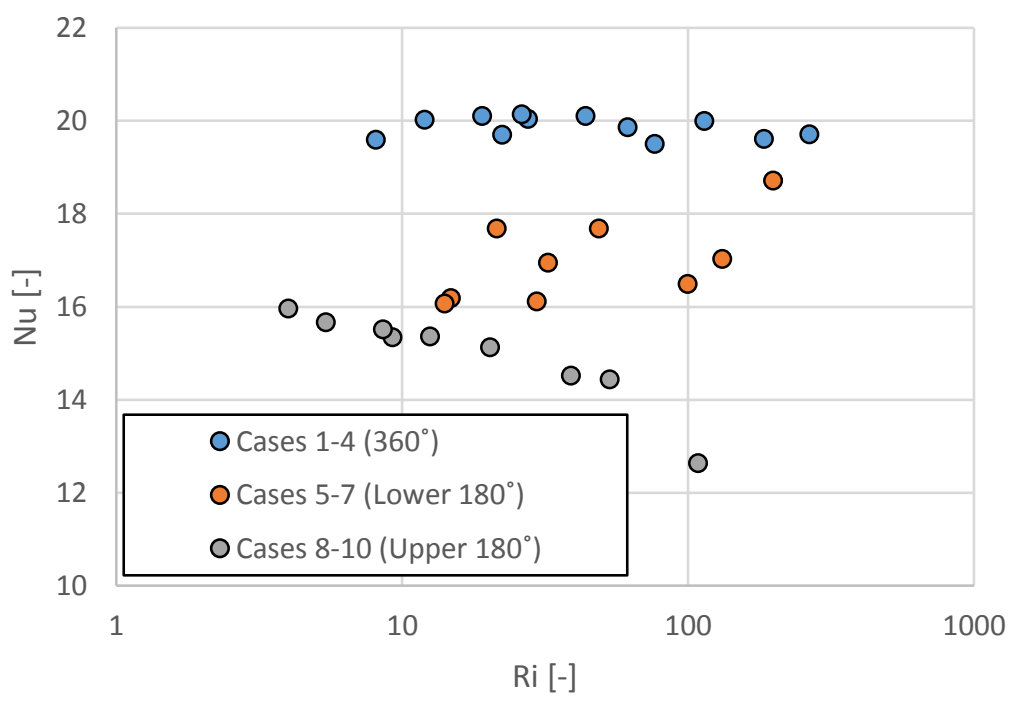

Figure 6-51: Nusselt number vs Richardson number for $360^{\circ}$, lower $180^{\circ}$ and upper $180^{\circ}$ cases 
Figure 6-52 shows the relationship between the Nusselt number and the product of the Richardson number and the Reynolds number to the power of 2.165. Figure 6-45 and Figure 6-51 clearly show the effect the Reynolds number has on the Richardson number. By introducing the Reynolds number into the equation, the data is far more condensed and a correlation can be created from the fitted curve.

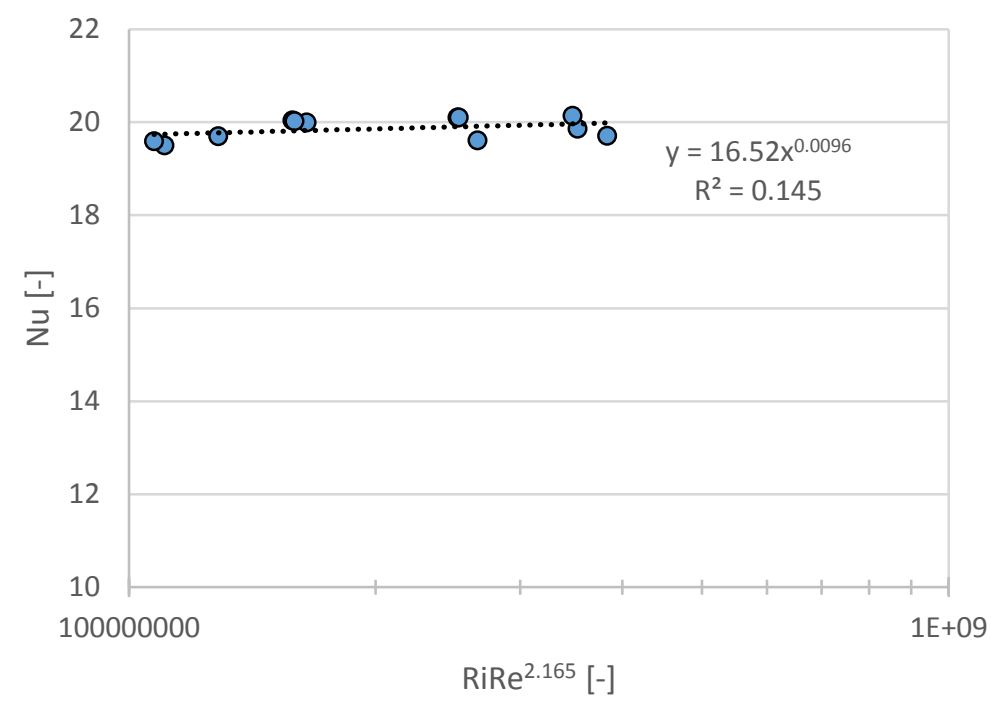

Figure 6-52: Trendline fit for $360^{\circ}$ heating cases

From the equation of the fitted curve, Equation 6.1 is found. Which is simplified to give the final correlation for $360^{\circ}$ heating cases as Equation 6.2. The correlation had a maximum error of $1.5 \%$ when compared to the experimental data. The average error for all the data was $0.98 \%$.

$$
\begin{gathered}
\mathrm{Nu}=16.52\left(\operatorname{RiRe}^{2.165}\right)^{0.0096} \\
\mathrm{Nu}=16.52\left(\mathrm{Ri}^{0.0096} \mathrm{Re}^{0.020784}\right)
\end{gathered}
$$

Figure 6-53 shows the fitted curve for the lower $180^{\circ}$ heating cases. Figure 6-46 and Figure 6-51 show the impact the Reynolds number has on the Richardson number relationship. However, unlike the $360^{\circ}$ heating case, the Reynolds number also affected the Nusselt numbers for the lower $180^{\circ}$ heating cases. With the Nusselt number increasing while the Reynolds number decreased. This meant that as well as adding the Reynolds number to the equation, the Prandtl number was also needed to account for the effect the change in Reynolds number had on the Nusselt number. 


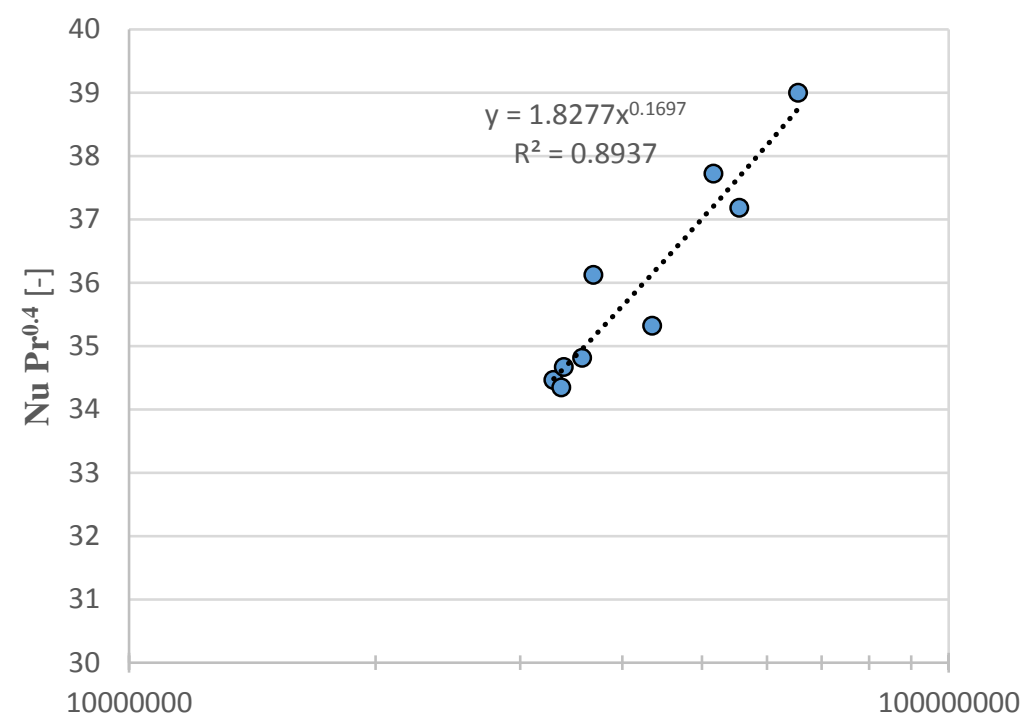

$\operatorname{Ri} \operatorname{Re}^{1.94}[-]$

Figure 6-53: Trendline fit for lower $180^{\circ}$ heating cases

The fitted curve equation is used to form Equation 6.3. Once simplified, Equation 6.4 gives the correlation for finding the Nusselt number for lower $180^{\circ}$ heating cases. The maximum error between the correlation and the experimental data is $2.7 \%$, with an average error of $1.3 \%$

$$
\begin{gathered}
\mathrm{NuPr}^{0.4}=1.8277\left(\operatorname{RiRe}^{1.94}\right)^{0.1697} \\
\mathrm{Nu}=\frac{1.8277\left(\mathrm{Ri}^{0.1697} \mathrm{Re}^{0.3292}\right)}{\operatorname{Pr}^{0.4}}
\end{gathered}
$$

Figure 6-54 shows the curve fitted to the data for the upper $180^{\circ}$ heating cases. Similar to the lower $180^{\circ}$ heating cases, Figure 6-47 shows how the Reynolds number affects both the Richardson number and Nusselt number. Unlike the lower $180^{\circ}$ heating cases, the Nusselt number increases as the Reynolds number increases for the upper heating cases. Therefore, the Reynolds number and Prandtl number are used to condense the data to allow for a more accurate correlation to be formed. 


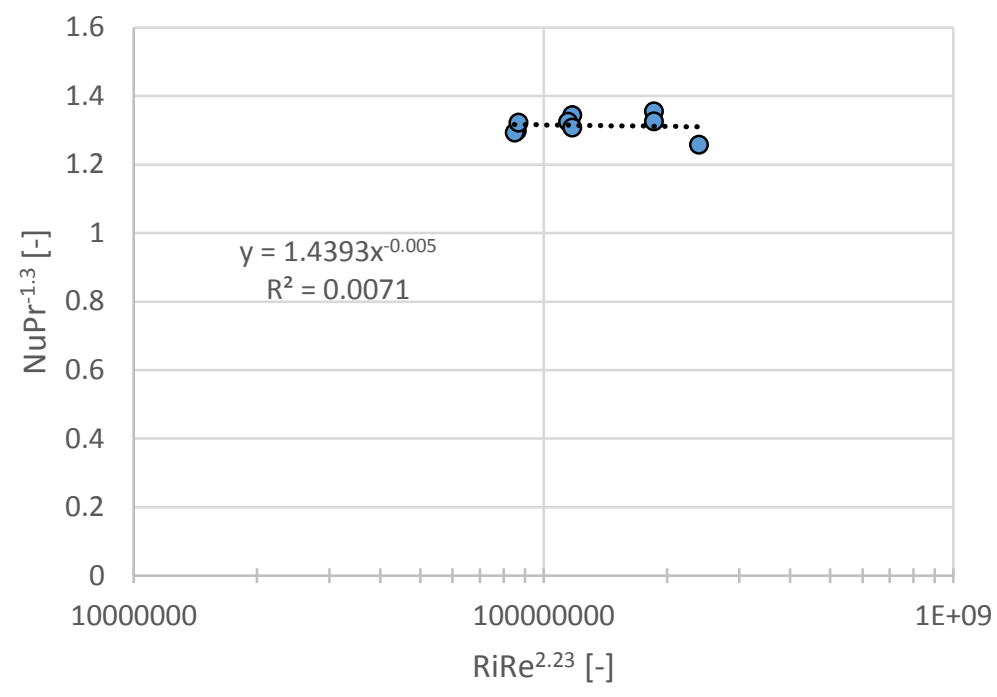

Figure 6-54: Trendline fit for upper $180^{\circ}$ heating cases

The fitted curve in Figure 6-54 is used to obtain Equation 6.5. The simplified equation give Equation 6.6 which is the correlation for the upper $180^{\circ}$ heating cases. The maximum error between the correlation and the experimental data is $3.7 \%$, with an average error of $1.7 \%$.

$$
\begin{aligned}
& \mathrm{NuPr}^{-1.3}=1.4395\left(\operatorname{RiRe}^{2.23}\right)^{-0.005} \\
& \mathrm{Nu}=1.4395\left(\frac{\operatorname{Pr}^{1.3}}{\mathrm{Ri}^{0.005} \times \mathrm{Re}^{0.01115}}\right)
\end{aligned}
$$

\subsection{Summary of Chapter}

In this chapter the validation and verification of the test procedure and data reduction methods for different intensity and positioning of the heat flux around a horizontal circular tube were presented. Followed by the experimental results, and lastly, tentative correlations which could be used to estimate the average heat transfer coefficient in terms of the Reynolds number, Prandtl number and the Richardson number.

The first verifications that were performed included the testing of the individual heating elements. These verification were used to ensure that each heating element was producing the same heat as the others. After checking the temperature difference between the inlet and outlet temperatures, it was seen that the heating elements were performing within $2 \%$ of each other. The axial wetted wall temperature profiles were then provided to ensure that the heating elements were heating evenly along their lengths. To ensure the tests were being performed in the laminar flow regime. The Reynolds number where the onset of transition occurs was checked. Nusselt number versus Reynolds number and Colburn J-factor versus Reynolds number plots were used to find this critical Reynolds number. This Reynolds number was around 3000.The experimental results were then compared to previous works. This was done for correlations in the laminar regime, the turbulent regime and also local Nusselt number correlations were checked. 
Temperature profiles at an average axial cross section for the different heating configurations were then provided. These temperature profiles allowed for the effects of the different heating configurations to be visualised. The left and right $180^{\circ}$ heating cases temperature profiles, as well as a comparison of their Nusselt number vs Reynolds number plots were used to visually show the symmetry that was expected between the left and right hemispheres.

Cases with the same heat fluxes were then investigated. This data showed the impact of the different heating configurations on the Nusselt numbers obtained. The difference between the heating cases is mainly due to the difference in buoyancy forces and secondary flow intensity depending on which portion of the pipe is heated. This assumption was then verified by plotting the Richardson number at different Reynolds numbers for the different cases. The graphs showed a direct correlation between the Richardson numbers and the associated Nusselt numbers. Cases with the same heat transfer rates were then tested and similar results to those for the cases with the same heat fluxes were observed.

The impact of the heat flux on the different cases was then investigated. This was done by testing cases with the same heating configuration at different heat fluxes. It was observed that the heat flux had very little impact on the $360^{\circ}$ heating cases, with a maximum error of $2 \%$ between the different cases. The lower $180^{\circ}$ cases did show Nusselt number changes as the heat flux was changed. The higher the heat flux the higher the Nusselt numbers produced. The upper $180^{\circ}$ cases responded very similarly to the $360^{\circ}$ heating cases. There was a $5 \%$ maximum difference between any of the upper $180^{\circ}$ cases Nusselt numbers at the same Reynolds number.

The effect of the Richardson number on the Nusselt number was then investigated. The $360^{\circ}$ heating cases showed that the Nusselt number remained fairly constant with the change in Richardson number. The lower $180^{\circ}$ heating cases showed there was an increase in the Nusselt number with an increase in the Richardson number. The upper $180^{\circ}$ cases showed the opposite effect with the Nusselt number decreasing as the Richardson number was increased. Plotting the Nusselt number versus Richardson number data at different Reynolds numbers reiterated the fact that the Richardson number decreases with an increase in the Reynolds number.

In order to see the influence of the heating position on the Richardson number, the Richardson number was plotted against the central heating position (in degrees) of the different heating configurations at a heat flux of $6631 \mathrm{~W} / \mathrm{m}^{2}$. The upper $180^{\circ}$ heating case showed the lowest Richardson number. The lower $180^{\circ}$, right and slanted heated cases had very similar Richardson numbers. The Nusselt numbers of the same cases were also plotted against the central heating position to show the influence of the heating position on the Nusselt number. The Nusselt number was seen to increase the more the central heating point was moved to the bottom of the tube (gravitationally speaking).

Then correlations for the $360^{\circ}$, lower $180^{\circ}$ and upper $180^{\circ}$ heating cases were then found. The Nusselt number correlation for the $360^{\circ}$ heating case was a function of the Richardson number and the Reynolds number. The correlation had an average error of $0.98 \%$. The Nusselt number correlation for the lower $180^{\circ}$ heating case was a function of the Richardson number, Reynolds number and the PrandtI number. The correlation had an average error of $1.3 \%$. The Nusselt number correlation for the upper $180^{\circ}$ heating case was a function of the Richardson number, Reynolds number and Prandtl number. The correlation had an average error of $1.7 \%$. 


\section{Summary, Conclusion and Recommendations}

\subsection{Summary}

Based on the literature review it was found that a need existed to quantify the effect that the intensity and position of non-uniform heat flux have on the effective internal heat transfer coefficient, and to determine whether a link existed between buoyancy driven secondary flow and heat transfer enhancement. An experimental setup to investigate the effect of non-uniform heat flux was designed, constructed, verified and validated. The test section consisted of an electrically heated horizontal stainless steel tube which had a length of $2 \mathrm{~m}$ and an inner diameter of $27.8 \mathrm{~mm}$. The inlet flow condition was fully hydrodynamically developed flow. Different heat flux intensities and positions of the imposed heat flux on the circumference of the tube were investigated. Steady state experimental data was gathered for flow conditions in the laminar flow regime where secondary flow effects were expected to be significant. Raw data was analyzed to obtain the average and local heat transfer coefficients, as well as other important indicators. The processed data was summarized in trends and tentative heat transfer coefficient correlations were produced.

\subsection{Conclusions}

- The average Nusselt numbers for the uniform heating cases $\left(360^{\circ}\right.$ heating) remained fairly constant throughout the Reynolds range tested. The lower $180^{\circ}$ cases showed the average Nusselt number decrease with an increase in Reynolds number and the average Nusselt number of the upper $180^{\circ}$ cases increased with an increase in Reynolds number.

- Uniform heating cases $\left(360^{\circ}\right.$ heating) were seen to have higher average Nusselt numbers when compared to their lower $180^{\circ}$ and upper $180^{\circ}$ heating case counterparts. This was due to the uniform heating cases having more heat input.

- When comparing the average Nusselt numbers of the lower $180^{\circ}$ and upper $180^{\circ}$ heating cases. The lower $180^{\circ}$ cases always have higher average Nusselt numbers. This was expected due to the increase in buoyancy forces and secondary flow for the lower $180^{\circ}$ cases. The aforementioned trend remained true irrespective of whether the heat flux or heat transfer rate were held constant.

- The intensity of the heat flux had very little effect on the average Nusselt numbers for the uniform heating and upper $180^{\circ}$ heating cases. Whereas, the average Nusselt numbers were seen to increase with an increase in heat flux intensity for the lower $180^{\circ}$ heating cases.

- The Richardson number had very little effect on the average Nusselt numbers for uniform heating cases. Lower $180^{\circ}$ heating cases, showed that the average Nusselt numbers increased as the Richardson number increased and the average Nusselt numbers of the upper $180^{\circ}$ cases decreased with an increase in Richardson number.

- When comparing $180^{\circ}$ heating cases with the same heat flux the Nusselt number increases as the heat flux position is moved towards the bottom of the pipe.

- Correlations were formed for the $360^{\circ}$ heating, the lower $180^{\circ}$ heating and the upper $180^{\circ}$ heating cases.

- The $360^{\circ}$ heating cases Nusselt number correlation was a function the Richardson number and Reynolds number. The lower and upper $180^{\circ}$ heating cases Nusselt number correlations were functions of the Richardson number, the Reynolds number and the Prandtl number.

- The $360^{\circ}$ heating case correlation had an average error of $0.98 \%$, the lower $180^{\circ}$ heating case correlation had an average error of $1.3 \%$ and the upper $180^{\circ}$ heating case correlation had an average error of $1.7 \%$. 


\subsection{Recommendations}

It is recommended that the following work be done to carry on the research found here:

- Testing the effect of circumferentially non-uniform heat flux on the transitional and turbulent regimes.

- More test sections should be constructed in order to test the effect of the L/D ratio on the effect of the circumferentially non-uniform heat flux. Test sections where $L / D>70$ should be prioritised due to more real world applications.

- Although the heat flux in this study is circumferentially non-uniform, the distribution of the heat flux is uniform in that segment. More realistic heat flux mapping on the sections of the pipe should be investigated.

- More of an investigation into the impact of non-uniform heat flux on local Nusselt numbers

- A wider range of convection can be investigated. Having natural dominant convection, mixed convection and forced dominant convection data would allow for a universal correlation to be developed which would be able to be applied for all cases. 


\section{References}

BERGLES, A. \& SIMONDS, R. 1971. Combined forced and free convection for laminar flow in horizontal tubes with uniform heat flux. International Journal of Heat and Mass Transfer, 14, 1989-2000.

BROWN, A. \& THOMAS, M. 1965. Combined free and forced convection heat transfer for laminar flow in horizontal tubes. Journal of Mechanical Engineering Science, 7, 440-448.

CHANG, C., LI, X. \& ZHANG, Q. Q. 2014. Experimental and Numerical Study of the Heat Transfer Characteristics in Solar Thermal Absorber Tubes with Circumferentially Non-uniform Heat Flux. Energy Procedia, 49, 305-313.

CHOU, F. \& HWANG, G. 1988. Numerical analysis of the Graetz problem with natural convection in a uniformly heated horizontal tube. International Journal of Heat and Mass Transfer, 31, 1299-1308.

DEPEW, C. A. \& AUGUST, S. E. 1971. Heat Transfer Due to Combined Free and Forced Convection in a Horizontal and Isothermal Tube. Journal of Heat Transfer, 93, 380-384.

DIRKER, J., MEYER, J. P. \& GARACH, D. V. 2014. Inlet flow effects in micro-channels in the laminar and transitional regimes on single-phase heat transfer coefficients and friction factors. International Journal of Heat and Mass Transfer, 77, 612-626.

DITTUS, F. W. \& BOELTER, L. M. K. 1985. Heat transfer in automobile radiators of the tubular type. International Communications in Heat and Mass Transfer, 12, 3-22.

DUNN, P. F. 2010. Measurement and Data Analysis for Engineering and Science. Second ed.: CRC Press.

ELATAR, A. \& SIDDIQUI, K. 2014. The effect of mixed convection on the structure of channel flow at low Reynolds numbers. International Journal of Heat and Fluid Flow, 46, 29-42.

GAULER, K. 1972. Warme- und Stoffubertragung an eine mitbewegte ebene Grenzflache bei Grenzschichtstomung, Dissertation Universitat Karlsruhe.

GHAJAR, A. J. \& TAM, L.-M. 1994. Heat transfer measurements and correlations in the transition region for a circular tube with three different inlet configurations. Experimental thermal and fluid science, 8, 79-90.

GNIELINSKI, V. 2010. 'G1 Heat Transfer in Pipe Flow' in VDI Heat Atlas, Berlin Heidelberg, SpringerVerlang.

HUANG, Z., LI, Z.-Y. \& TAO, W.-Q. 2015. Numerical study on combined natural and forced convection in the fully-developed turbulent region for a horizontal circular tube heated by non-uniform heat flux.

KUPPER, A., HAUPTMANN, E. \& IQBAL, M. 1969. Combined free and forced convection in a horizontal tube under uniform heat flux. Solar Energy, 12, 439-446.

LI, Z.-Y., HUANG, Z. \& TAO, W.-Q. 2016. Three-dimensional numerical study on fully-developed mixed laminar convection in parabolic trough solar receiver tube. Energy, 113, 1288-1303.

LIN, W. L. \& LIN, T. F. 1996. Experimental study of unstable mixed convection of air in a bottom heated horizontal rectangular duct. International Journal of Heat and Mass Transfer, 39, 1649-1663. 
MOHAMMED, H. A. \& SALMAN, Y. K. 2007. Experimental investigation of mixed convection heat transfer for thermally developing flow in a horizontal circular cylinder. Applied thermal engineering, 27, 1522-1533.

MORCOS, S. \& BERGLES, A. 1975. Experimental investigation of combined forced and free laminar convection in horizontal tubes. Journal of Heat Transfer, 97, 212-219.

OKAFOR, I. F., DIRKER, J. \& MEYER, J. P. Numerical study of heat transfer characteristics for different solar flux distributions on linear Fresnel collector absorber tubes in laminar flow. Proceedings of the 15th International Heat Transfer Conference, Kyoto, paper IHTC15-9221, 2014. 11-15.

OLIVER, D. 1962. The effect of natural convection on viscous-flow heat transfer in horizontal tubes. Chemical Engineering Science, 17, 335-350.

POPIEL, C. \& WOJTKOWIAK, J. 1998. Simple formulas for thermophysical properties of liquid water for heat transfer calculations (from $0 \mathrm{C}$ to $150 \mathrm{C}$ ). Heat transfer engineering, 19, 87-101.

SIEDER, E. N. \& TATE, G. E. 1936. Heat Transfer and Pressure Drop of Liquids in Tubes. Industrial \& Engineering Chemistry, 28, 1429-1435.

YASUO, M., KOZO, F., SHINOBU, T. \& MASAKUNI, N. 1966. Forced convective heat transfer in uniformly heated horizontal tubes 1st report-Experimental study on the effect of buoyancy. International Journal of Heat and Mass Transfer, 9, 453-463.

\section{Appendix A}

\subsection{Uncertainty Analysis}

\subsubsection{Introduction}

All measured experimental results inevitably have some form of inaccuracies. These come from the bias and the precision in the system. The bias is the accuracy of the measurement instrument and is specified by the manufacturer. The precision is the standard deviation of the data in the data set itself.

\subsubsection{Thermocouples}

The thermocouples were calibrated using the in situ method. This meant that two PT-100 temperature probes were used with the temperature ranging between $20^{\circ} \mathrm{C}$ and $65^{\circ} \mathrm{C}$. Increments of approximately $2.5^{\circ} \mathrm{C}$ were used when gathering data, therefore eighteen data points were captured. The PT-100 probes have an accuracy of $0.1^{\circ} \mathrm{C}$. The bias and precision mentioned earlier are used to find the uncertainty of a single measurement (Dunn, 2010).

The following equations were used to find the precision of each of the thermocouples.

$$
\begin{gathered}
S_{x x}=\sum_{i=1}^{N}\left(x_{i}-\bar{x}\right)^{2} \\
S_{x y}=\sum_{i=1}^{N}\left(x_{i}-\bar{x}\right)\left(y_{i}-\bar{y}\right) \\
b=\frac{S_{x y}}{S_{x x}}
\end{gathered}
$$




$$
\begin{gathered}
a=\bar{y}-b \bar{x} \\
S_{y x}=\sqrt{\frac{\sum_{i=1}^{N}\left(y_{i}-y_{c i}\right)^{2}}{N-2}} \\
\delta x=\frac{\delta y}{n}
\end{gathered}
$$

The overall uncertainty of each uncertainty thermocouple was then found using:

$$
\delta x=\left(b_{i}^{2}+p_{p_{i}}^{2}\right)^{\frac{1}{2}}
$$

Where the bias was determined using the calibration curves and the comparison to the PT-100 probe.

The minimum uncertainty $0.1^{\circ} \mathrm{C}$ was seen to be and the maximum uncertainty was $0.15^{\circ} \mathrm{C}$.

\subsubsection{Coriolis Flow Meters}

The Coriolis flow meter with a measuring range of 0 to $0.604 \mathrm{~kg} / \mathrm{s}$ had an accuracy of $0.05 \%$. Therefore the CFM025 had a capacity of $2174 \mathrm{l} / \mathrm{h}$ and accuracy of 1.087 .

\subsubsection{Fluid Properties}

The uncertainty of the fluid properties can be calculated using the equations put forward by Popiel and Wojtkowiak (1998). The relevant properties are provided in the table below.

\begin{tabular}{|l|c|}
\hline Property & Uncertainty \\
\hline$C_{p}[\mathrm{~J} / \mathrm{kgK}]$ & $0.04 \%$ \\
\hline $\boldsymbol{\mu}[\mathrm{kg} / \mathrm{ms}]$ & $1 \%$ \\
\hline $\boldsymbol{k}[\mathrm{W} / \mathrm{mK}]$ & $2 \%$ \\
\hline $\boldsymbol{\rho}\left[\mathrm{kg} / \mathrm{m}^{3}\right]$ & $0.004 \%$ \\
\hline $\boldsymbol{P r}[-]$ & $2.3 \%$ \\
\hline
\end{tabular}

Table 9-1: Fluid Properties Uncertainties(Popiel and Wojtkowiak, 1998)

\subsubsection{Dimensions}

The uncertainties associated with the dimensions used are from the equipment used to measure the different dimensions. The thickness and diameters were measured with a vernier calliper and the length was measured with a measuring tape.

\begin{tabular}{|l|c|}
\hline Dimension & Accuracies \\
\hline $\boldsymbol{t}[\mathrm{m}]$ & $20[\mu \mathrm{m}]$ \\
\hline $\boldsymbol{D}[\mathrm{m}]$ & $20[\mu \mathrm{m}]$ \\
\hline $\boldsymbol{L}[\mathrm{m}]$ & $1[\mathrm{~mm}]$ \\
\hline
\end{tabular}

Table 9-2: Accuracy of Dimensions

\subsubsection{Inlet and Outlet Average Temperatures}

The uncertainties of the bulk inlet and outlet temperatures are given by the equations below. These are the uncertainties of the temperatures measured after the mixer at the inlet (before the development length) and after the mixer at the outlet (after the test section). 


$$
\begin{gathered}
\delta \bar{T}_{\text {Bin }}=\left[\left(\frac{\delta T_{1}}{4}\right)^{2}+\left(\frac{\delta T_{2}}{4}\right)^{2}+\left(\frac{\delta T_{3}}{4}\right)^{2}+\left(\frac{\delta T_{4}}{4}\right)^{2}\right]^{\frac{1}{2}} \\
\delta \bar{T}_{\text {Bout }}=\left[\left(\frac{\delta T_{45}}{4}\right)^{2}+\left(\frac{\delta T_{46}}{4}\right)^{2}+\left(\frac{\delta T_{47}}{4}\right)^{2}+\left(\frac{\delta T_{48}}{4}\right)^{2}\right]^{\frac{1}{2}}
\end{gathered}
$$

\subsubsection{Inner Wall Axial Average Temperature}

The uncertainties of the average temperatures of the inner wall along the axis are given by the equations below:

$$
\begin{gathered}
\delta \bar{T}_{\text {Win }}=\left[\left(\frac{\delta T_{5}}{8}\right)^{2}+\left(\frac{\delta T_{6}}{8}\right)^{2}+\left(\frac{\delta T_{7}}{8}\right)^{2}+\left(\frac{\delta T_{8}}{8}\right)^{2}\left(\frac{\delta T_{9}}{8}\right)^{2}+\left(\frac{\delta T_{10}}{8}\right)^{2}+\left(\frac{\delta T_{11}}{8}\right)^{2}+\left(\frac{\delta T_{12}}{8}\right)^{2}\right]^{\frac{1}{2}} \\
\delta \bar{T}_{\text {Wout }}=\left[\left(\frac{\delta T_{37}}{8}\right)^{2}+\left(\frac{\delta T_{38}}{8}\right)^{2}+\left(\frac{\delta T_{39}}{8}\right)^{2}+\left(\frac{\delta T_{40}}{8}\right)^{2}\left(\frac{\delta T_{41}}{8}\right)^{2}+\left(\frac{\delta T_{42}}{8}\right)^{2}+\left(\frac{\delta T_{43}}{8}\right)^{2}+\left(\frac{\delta T_{44}}{8}\right)^{2}\right]^{\frac{1}{2}}
\end{gathered}
$$

\subsubsection{Bulk Fluid Uncertainty}

The uncertainty of the bulk fluid temperature was calculated by:

$$
\delta T_{B}=\left[\left(\frac{\delta \bar{T}_{\text {Bin }}}{2}\right)^{2}+\left(\frac{\delta \bar{T}_{\text {Bout }}}{2}\right)^{2}\right]^{\frac{1}{2}}
$$

\subsubsection{Wall Temperature Uncertainty}

The uncertainty of the wall temperature was determined using:

$$
\delta T_{W}=\left[\left(\frac{\delta \bar{T}_{\text {Win }}}{2}\right)^{2}+\left(\frac{\delta \bar{T}_{\text {Wout }}}{2}\right)^{2}\right]^{\frac{1}{2}}
$$

\subsubsection{Heated Surface Area}

The uncertainty of the heated surface area was determined using:

$$
\begin{gathered}
A_{s}=\pi D_{i} L \\
\delta A_{s}=\left[\left(\pi L \delta D_{i}\right)^{2}+\left(\pi D_{i} \delta L\right)^{2}\right]^{\frac{1}{2}}
\end{gathered}
$$

\subsubsection{Applied Heat}

The uncertainty of the heat applied to the system was determined by: 


$$
\begin{gathered}
\dot{Q}=I V \\
\delta \dot{Q}=\left[(I \delta V)^{2}+(V \delta I)^{2}\right]^{\frac{1}{2}}
\end{gathered}
$$

\subsubsection{Heat Flux}

The uncertainty of the heat flux is determined by the following equations and uses the uncertainties of the applied heat and heated surface area.

$$
\begin{gathered}
\dot{q}=\frac{\dot{Q}}{A_{s}} \\
\delta \dot{q}=\left[\left(\frac{\delta \dot{Q}}{A_{s}}\right)^{2}+\left(-\left(\frac{\dot{Q} \delta A_{s}}{A_{S}^{2}}\right)\right)^{2}\right]^{\frac{1}{2}}
\end{gathered}
$$

\subsubsection{Heat Transfer Coefficient}

The uncertainty of the heat transfer coefficient uses the uncertainty of the applied heat flux, the uncertainty of the wall temperature and the uncertainty of the bulk fluid temperature.

$$
\begin{gathered}
h=\frac{\dot{q}}{\left(\bar{T}_{W}-\bar{T}_{B}\right)} \\
\delta h=\left[\left(\frac{\delta \dot{q}}{\bar{T}_{W}-\bar{T}_{B}}\right)^{2}+\left(-\frac{\dot{q} \delta T_{W}}{\left(\bar{T}_{W}-\bar{T}_{B}\right)^{2}}\right)^{2}+\left(\frac{\dot{q} \delta T_{B}}{\left(\bar{T}_{W}-\bar{T}_{B}\right)^{2}}\right)^{2}\right]^{\frac{1}{2}}
\end{gathered}
$$

\subsubsection{Nusselt Number}

The uncertainty of the Nusselt number uses the uncertainty of the heat transfer coefficient, the uncertainty of the inner diameter and the uncertainty of the thermal conductivity, and is found using the following equations:

$$
\begin{gathered}
N u=\frac{h D_{i}}{k} \\
\delta N u=\left[\left(\frac{D_{i} \delta h}{k}\right)^{2}+\left(\frac{h \delta D_{i}}{k}\right)^{2}+\left(-\frac{h D_{i} \delta k}{k^{2}}\right)^{2}\right]^{\frac{1}{2}}
\end{gathered}
$$

\subsubsection{Local Nusselt Number}

$$
\begin{gathered}
N u_{\text {local }}=\frac{h_{\text {local }} D_{i}}{k_{\text {local }}} \\
\delta N u_{\text {local }}=\left\{\left(\frac{\partial N u_{\text {local }}}{\partial h_{\text {local }}} \delta h_{\text {local }}\right)^{2}+\left(\frac{\partial N u_{\text {local }}}{\partial D_{i}} \delta D_{i}\right)^{2}+\left(\frac{\partial N u_{\text {local }}}{\partial k_{\text {local }}} \delta k_{\text {local }}\right)^{2}\right\}^{\frac{1}{2}} \\
=\left\{\left(\frac{D_{i}}{k_{\text {local }}} \delta h_{\text {local }}\right)^{2}+\left(\frac{h_{\text {local }}}{k_{\text {local }}} \delta D_{i}\right)^{2}+\left(-\frac{h_{\text {local }} D_{i}}{k_{\text {local }}^{2}} \delta k_{\text {local }}\right)^{2}\right\}^{\frac{1}{2}}
\end{gathered}
$$




\subsubsection{Conclusion}

This chapter contains all the equations used for the different uncertainties and accuracies of the instruments used, the fluid properties, dimensions and the calculated parameters used in the data reduction and results sections. The results from these equations can be found in the data reduction chapter found in the main body of the dissertation. 


\section{Appendix B}

\subsection{Calibration Polynomials}

This appendix contains the constants for the calibration polynomials $\left(y x^{2}+u x+c\right)$ used to calibrate the thermocouples.

Table 10-1: Calibration polynomial constants part 1

\begin{tabular}{|c|c|c|c|c|c|c|c|}
\hline & $y$ & $u$ & $c$ & & $y$ & $u$ & $c$ \\
\hline $\mathrm{m}=0, \mathrm{TC} 1$ & .00000567 & 001055 & .05703 & $=2, \mathrm{TC} 17$ & $.5 \mathrm{E}-05$ & 003726 & 11 \\
\hline$m=0$ & & & & 18 & & & \\
\hline $\mathrm{m}=0$, & & & & 19 & & & \\
\hline $\mathrm{m}=0, \mathrm{TC} 4$ & 6.01 & 1 & & $=2, \mathrm{~T}$ & & & \\
\hline $\mathrm{m}=1$, & & & & 1 & & & \\
\hline$m=1$, & & & & $\mathrm{m}=3$ & & & \\
\hline$m=1$, & -1 & & & $\mathrm{~m}=3$, & & & \\
\hline$m=1$, & & & & 24 & & & \\
\hline $\mathrm{m}=1, \mathrm{TC} 9$ & 55 & & & $m=3$, & & & 21 \\
\hline$m=1$, & & & & $m=3$ & & & \\
\hline$m=1$, & & & & $\mathrm{m}=3$, & & & \\
\hline$m=1, T C 12$ & -0. & 6 & & $m-2$ & & & \\
\hline $\mathrm{m}=2, \mathrm{TC} 13$ & & & & $\mathrm{~m}=4$ & & & 23 \\
\hline$m=2, T C 14$ & & & & $n=4, T$ & & & \\
\hline$m=2, T C 15$ & & & & & & & \\
\hline$m=2$, TC16 & $-3.69082 \mathrm{E}-05$ & 0.003814 & -0.61609 & $m=4, T C 32$ & $-1 \mathrm{E}-05$ & 0.001381 & -0.11884 \\
\hline
\end{tabular}


Table 10-2: Calibration polynomial constants part 2

\begin{tabular}{|c|c|c|c|c|c|c|c|}
\hline & $y$ & $u$ & C & & $y$ & $u$ & C \\
\hline$m=4$, TC33 & $.36078 \mathrm{E}-05$ & 003366 & .19041 & 1=6, TC49 & $-3.7 E-05$ & .0043 & 3419 \\
\hline & 05 & & & 50 & & 064 & \\
\hline$m=4$, & 05 & & & & & & \\
\hline$m=4, T C 36$ & $-2.21056 \mathrm{E}-05$ & & & $n=6$, TC52 & & & \\
\hline$=5$, & -3.3 & 81 & & 53 & -2.5 & & 571 \\
\hline$m=5$, & & & & & & & \\
\hline$m=5$, & -1. & & & $n=7$ & & & 154 \\
\hline$m=5$ & -2.5 & & & $m=7$ & -9 & 04 & \\
\hline$m=5$, & & & & 7 & & & \\
\hline$m=5$, & -3.5 & & & 8 & & & \\
\hline$m=5$ & -1. & & & $m=7$ & $-3 \mathrm{E}$ & & \\
\hline$m=5, \mathrm{TC} 44$ & -2.4 & & & 50 & $-1.5 \mathrm{E}-05$ & 32 & \\
\hline$m=6, T C 45$ & -3.34 & & & $\mathrm{~m}=8$ & & & 2416 \\
\hline$m=6, T C 46$ & -2.33 & & & $m=8$ & -05 & & \\
\hline$m=6, T C 47$ & -4.3 & & & 63 & $-2.5 \mathrm{E}-05$ & & \\
\hline$m=6, T C 48$ & -0.000023099 & 0.002722 & -0.425 & $m=8, T C 64$ & $-2.8 \mathrm{E}-05$ & 0.002881 & -0.53276 \\
\hline
\end{tabular}


